\title{
Interval-Valued Intuitionistic Hesitant Fuzzy Aggregation Operators and Their Application in Group Decision-Making
}

\author{
Zhiming Zhang \\ College of Mathematics and Computer Science, Hebei University, Baoding, Hebei 071002, China \\ Correspondence should be addressed to Zhiming Zhang; zhimingzhang@ymail.com
}

Received 4 March 2013; Revised 30 May 2013; Accepted 17 June 2013

Academic Editor: Luis Javier Herrera

Copyright (c) 2013 Zhiming Zhang. This is an open access article distributed under the Creative Commons Attribution License, which permits unrestricted use, distribution, and reproduction in any medium, provided the original work is properly cited.

\begin{abstract}
Hesitant fuzzy sets, permitting the membership of an element to be a set of several possible values, can be used as an efficient mathematical tool for modelling people's hesitancy in daily life. In this paper, we extend the hesitant fuzzy set to interval-valued intuitionistic fuzzy environments and propose the concept of interval-valued intuitionistic hesitant fuzzy set, which allows the membership of an element to be a set of several possible interval-valued intuitionistic fuzzy numbers. The aim of this paper is to develop a series of aggregation operators for interval-valued intuitionistic hesitant fuzzy information. Then, some desired properties of the developed operators are studied, and the relationships among these operators are discussed. Furthermore, we apply these aggregation operators to develop an approach to multiple attribute group decision-making with interval-valued intuitionistic hesitant fuzzy information. Finally, a numerical example is provided to illustrate the application of the developed approach.
\end{abstract}

\section{Introduction}

In many practical problems, when defining the membership degree of an element, the difficulty of establishing the membership degree is not because we have a margin of error (as in intuitionistic fuzzy sets [1] and interval-valued fuzzy sets [2]) or some possibility distribution (as in type 2 fuzzy sets [3]) on the possibility values, but because we have several possible numerical values. To deal with such cases, Torra [4] introduced the concept of hesitant fuzzy set to permit the membership of an element to be a set of several possible values between 0 and 1 , which can depict the human's hesitance more objectively and precisely.

It should be noted that hesitant fuzzy sets permit the membership of an element to be a set of several possible values. All these possible values are crisp real numbers that belong to $[0,1]$. However, in the process of some practical decision-makings, sometimes, due to the time pressure and lack of knowledge or data or the decision makers' (DMs) limited attention and information processing capacities, the DMs cannot provide their evaluations with a single numerical value, a margin of error, some possibility distribution on the possible values, several possible numerical values, several possible interval numbers, or several possible intuitionistic fuzzy numbers but several possible interval-valued intuitionistic fuzzy numbers. For example, to get a reasonable decision result, a decision organization, which contains a lot of decision makers, is required to estimate the degree that an alternative satisfies an attribute. Suppose there are three cases: some decision makers provide $([0.5,0.7],[0.2,0.3])$, some assign $([0.2,0.3],[0.5,0.6])$, and the others provide $([0.4,0.6],[0.1,0.3])$, and these three parts cannot persuade each other to change their opinions. We can easily see that such cases cannot be dealt with by fuzzy sets [5], hesitant fuzzy sets, and their extensions, such as interval-valued fuzzy sets, intuitionistic fuzzy sets, interval-valued intuitionistic fuzzy sets, type 2 fuzzy sets, interval-valued hesitant fuzzy sets [6], and generalized hesitant fuzzy sets [7]. Thus, it is very necessary to introduce a new extension of hesitant fuzzy sets to address this issue. The aim of this paper is to present the notion of interval-valued intuitionistic hesitant fuzzy set, which extends the hesitant fuzzy set to intervalvalued intuitionistic fuzzy environments and permits the membership of an element to be a set of several possible interval-valued intuitionistic fuzzy numbers. Thus, intervalvalued intuitionistic hesitant fuzzy set is a very useful tool to deal with the situations in which the experts hesitate between several possible interval-valued intuitionistic fuzzy 
numbers to assess the degree to which an alternative satisfies an attribute. In the previous example, the degree to which the alternative satisfies the attribute can be represented by an interval-valued intuitionistic hesitant fuzzy set $\{([0.5,0.7]$, $[0.2,0.3]),([0.2,0.3],[0.5,0.6]),([0.4,0.6],[0.1,0.3])\}$. Moreover, in many multiple attribute group decision-making (MAGDM) problems, considering that the estimations of the attribute values are interval-valued intuitionistic hesitant fuzzy sets, it therefore is very necessary to give some aggregation techniques to aggregate the interval-valued intuitionistic hesitant fuzzy information. However, we are aware that the existing aggregation techniques have difficulty in coping with group decision-making problems with intervalvalued intuitionistic hesitant fuzzy information. Therefore, we in the current paper propose a series of aggregation operators for aggregating the interval-valued intuitionistic hesitant fuzzy information and investigate some properties of these operators. Then, based on these aggregation operators, we develop an approach to MAGDM with interval-valued intuitionistic hesitant fuzzy information. Moreover, we use a numerical example to show the application of the developed approach.

In order to do this, this paper is organized as follows. Section 2 introduces some concepts and properties of interval-valued intuitionistic hesitant fuzzy sets. In Section 3, we present a series of aggregation operators for interval-valued intuitionistic hesitant fuzzy information and examine the relationships among these aggregation operators. Section 4 develops an approach to group decisionmakings with interval-valued intuitionistic hesitant fuzzy information. In the sequel, the application of the developed approach in group decision-making problems is shown by an illustrative example in Section 5. The final section offers some concluding remarks.

\section{Preliminaries}

In this section, we will briefly introduce the basic notions of hesitant fuzzy sets [4], interval-valued intuitionistic fuzzy sets [8], and interval-valued intuitionistic hesitant fuzzy sets.

\subsection{Hesitant Fuzzy Sets and Hesitant Fuzzy Elements}

Definition 1 (see [4]). Let $X$ be a fixed set; a hesitant fuzzy set (HFS) on $X$ is given in terms of a function that when applied to $X$ returns a subset of $[0,1]$.

To be easily understood, we express the HFS by a mathematical symbol

$$
E=\left\{\left\langle x, h_{E}(x)\right\rangle \mid x \in X\right\}
$$

where $h_{E}(x)$ is a set of some values in $[0,1]$, denoting the possible membership degrees of the element $x \in X$ to the set $E$. For convenience, Xia and Xu [9] called $h=h_{E}(x)$ a hesitant fuzzy element (HFE) and $H$ the set of all HFEs.
Given three HFEs represented by $h, h_{1}$, and $h_{2}$, Torra [4] defined some operations on them, which can be described as

$$
\begin{gathered}
h^{c}=\{1-\gamma \mid \gamma \in h\}, \\
h_{1} \cup h_{2}=\left\{\gamma_{1} \vee \gamma_{2} \mid \gamma_{1} \in h_{1}, \gamma_{2} \in h_{2}\right\}, \\
h_{1} \cap h_{2}=\left\{\gamma_{1} \wedge \gamma_{2} \mid \gamma_{1} \in h_{1}, \gamma_{2} \in h_{2}\right\} .
\end{gathered}
$$

Furthermore, in order to aggregate hesitant fuzzy information, $\mathrm{Xia}$ and $\mathrm{Xu}$ [9] defined some new operations on the HFEs $h, h_{1}$, and $h_{2}$ :

$$
\begin{gathered}
h_{1} \oplus h_{2}=\left\{\gamma_{1}+\gamma_{2}-\gamma_{1} \gamma_{2} \mid \gamma_{1} \in h_{1}, \gamma_{2} \in h_{2}\right\}, \\
h_{1} \otimes h_{2}=\left\{\gamma_{1} \gamma_{2} \mid \gamma_{1} \in h_{1}, \gamma_{2} \in h_{2}\right\}, \\
h^{\lambda}=\left\{\gamma^{\lambda} \mid \gamma \in h\right\}, \\
\lambda h=\left\{1-(1-\gamma)^{\lambda} \mid \gamma \in h\right\} .
\end{gathered}
$$

To compare the HFEs, Xia and Xu [9] defined the following comparison laws.

Definition 2 (see [9]). For an $\operatorname{HFE} h, s(h)=\sum_{\gamma \in h} \gamma / \# h$ is called the score function of $h$, where \#h is the number of the elements in $h$. For two HFEs $h_{1}$ and $h_{2}$, if $s\left(h_{1}\right)>s\left(h_{2}\right)$, then $h_{1}>h_{2}$; if $s\left(h_{1}\right)=s\left(h_{2}\right)$, then $h_{1}=h_{2}$.

\subsection{Interval-Valued Intuitionistic Fuzzy Sets and Interval-Valued Intuitionistic Fuzzy Numbers}

Definition 3 (see [8]). Let $X$ be an ordinary nonempty set. An interval-valued intuitionistic fuzzy set $A$ in $X$ is an object that has the form

$$
A=\left\{\left\langle x, \mu_{A}(x), v_{A}(x)\right\rangle \mid x \in X\right\}
$$

where $\mu_{A}(x)=\left[\mu_{A}^{-}(x), \mu_{A}^{+}(x)\right] \subset[0,1]$ and $v_{A}(x)=$ $\left[\nu_{A}^{-}(x), v_{A}^{+}(x)\right] \subset[0,1]$ satisfy $0 \leq \mu_{A}^{+}(x)+v_{A}^{+}(x) \leq 1$ for all $x \in X$ and are, respectively, called the membership degree and the nonmembership degree of the element $x \in U$ to $A$.

$\mathrm{Xu}$ [10] called each pair $\left(\mu_{A}(x), \nu_{A}(x)\right)$ in $A$ an intervalvalued intuitionistic fuzzy number (IVIFN), and, for convenience, each IVIFN can be simply denoted by $\alpha=\left(\mu_{\alpha}, \nu_{\alpha}\right)$, where $\mu_{\alpha}=\left[\mu_{\alpha}^{-}, \mu_{\alpha}^{+}\right] \subset[0,1], \nu_{\alpha}=\left[\nu_{\alpha}^{-}, v_{\alpha}^{+}\right] \subset[0,1]$, and $\mu_{\alpha}^{+}+\nu_{\alpha}^{+} \leq 1$. Let $\Omega$ be the set of all IVIFNs.

$\mathrm{Xu}$ [10] introduced the following operational laws for IVIFNs. 
Definition 4 (see [10]). Let $\alpha=\left(\mu_{\alpha}, v_{\alpha}\right)=\left(\left[\mu_{\alpha}^{-}, \mu_{\alpha}^{+}\right],\left[v_{\alpha}^{-}, v_{\alpha}^{+}\right]\right)$, $\alpha_{1}=\left(\mu_{\alpha_{1}}, v_{\alpha_{1}}\right)=\left(\left[\mu_{\alpha_{1}}^{-}, \mu_{\alpha_{1}}^{+}\right],\left[v_{\alpha_{1}}^{-}, v_{\alpha_{1}}^{+}\right]\right)$, and $\alpha_{2}=\left(\mu_{\alpha_{2}}, v_{\alpha_{2}}\right)=$ $\left(\left[\mu_{\alpha_{2}}^{-}, \mu_{\alpha_{2}}^{+}\right],\left[\nu_{\alpha_{2}}^{-}, \nu_{\alpha_{2}}^{+}\right]\right)$be any three IVIFNs. Then,

$$
\begin{aligned}
& \alpha^{c}=\left(\left[\nu_{\alpha}^{-}, v_{\alpha}^{+}\right],\left[\mu_{\alpha}^{-}, \mu_{\alpha}^{+}\right]\right), \\
& \alpha_{1} \vee \alpha_{2}=\left(\left[\mu_{\alpha_{1}}^{-} \vee \mu_{\alpha_{2}}^{-}, \mu_{\alpha_{1}}^{+} \vee \mu_{\alpha_{2}}^{+}\right],\left[v_{\alpha_{1}}^{-} \wedge \nu_{\alpha_{2}}^{-}, v_{\alpha_{1}}^{+} \wedge v_{\alpha_{2}}^{+}\right]\right) \text {, } \\
& \alpha_{1} \wedge \alpha_{2}=\left(\left[\mu_{\alpha_{1}}^{-} \wedge \mu_{\alpha_{2}}^{-}, \mu_{\alpha_{1}}^{+} \wedge \mu_{\alpha_{2}}^{+}\right],\left[\nu_{\alpha_{1}}^{-} \vee v_{\alpha_{2}}^{-}, v_{\alpha_{1}}^{+} \vee v_{\alpha_{2}}^{+}\right]\right) \text {, } \\
& \alpha_{1} \oplus \alpha_{2}=\left(\left[\mu_{\alpha_{1}}^{-}+\mu_{\alpha_{2}}^{-}-\mu_{\alpha_{1}}^{-} \mu_{\alpha_{2}}^{-}, \mu_{\alpha_{1}}^{+}+\mu_{\alpha_{2}}^{+}-\mu_{\alpha_{1}}^{+} \mu_{\alpha_{2}}^{+}\right]\right. \text {, } \\
& \left.\left[v_{\alpha_{1}}^{-} v_{\alpha_{2}}^{-}, v_{\alpha_{1}}^{+} v_{\alpha_{2}}^{+}\right]\right) \text {, } \\
& \alpha_{1} \otimes \alpha_{2}=\left(\left[\mu_{\alpha_{1}}^{-} \mu_{\alpha_{2}}^{-}, \mu_{\alpha_{1}}^{+} \mu_{\alpha_{2}}^{+}\right]\right. \text {, } \\
& \left.\left[v_{\alpha_{1}}^{-}+v_{\alpha_{2}}^{-}-v_{\alpha_{1}}^{-} v_{\alpha_{2}}^{-}, v_{\alpha_{1}}^{+}+v_{\alpha_{2}}^{+}-v_{\alpha_{1}}^{+} v_{\alpha_{2}}^{+}\right]\right) \text {, } \\
& \lambda \alpha=\left(\left[1-\left(1-\mu_{\alpha}^{-}\right)^{\lambda}, 1-\left(1-\mu_{\alpha}^{+}\right)^{\lambda}\right],\left[\left(\nu_{\alpha}^{-}\right)^{\lambda},\left(\nu_{\alpha}^{+}\right)^{\lambda}\right]\right) \text {, } \\
& \lambda>0 \text {, } \\
& \alpha^{\lambda}=\left(\left[\left(\mu_{\alpha}^{-}\right)^{\lambda},\left(\mu_{\alpha}^{+}\right)^{\lambda}\right],\left[1-\left(1-v_{\alpha}^{-}\right)^{\lambda}, 1-\left(1-v_{\alpha}^{+}\right)^{\lambda}\right]\right) \text {, } \\
& \lambda>0 \text {. }
\end{aligned}
$$

Theorem 5 (see [10]). Let $\alpha=\left(\mu_{\alpha}, v_{\alpha}\right), \alpha_{1}=\left(\mu_{\alpha_{1}}, v_{\alpha_{1}}\right)$, and $\alpha_{2}=\left(\mu_{\alpha_{2}}, v_{\alpha_{2}}\right)$ be any three IVIFNs. Then, $\alpha_{1} \oplus \alpha_{2}, \alpha_{1} \otimes \alpha_{2}, \lambda \alpha$, and $\alpha^{\lambda}$ are also IVIFNs.

$\mathrm{Xu}[10]$ introduced the score function $s(\alpha)=(1 / 2)\left(\mu_{\alpha}^{-}-\right.$ $\left.v_{\alpha}^{-}+\mu_{\alpha}^{+}-v_{\alpha}^{+}\right)$to get the score of $\alpha$ and defined an accuracy function $h(\alpha)=(1 / 2)\left(\mu_{\alpha}^{-}+v_{\alpha}^{-}+\mu_{\alpha}^{+}+v_{\alpha}^{+}\right)$to evaluate the accuracy degree of $\alpha$. Xu [10] gave an order relation between two IVIFNs $\alpha_{1}$ and $\alpha_{2}$.

(1) If $s\left(\alpha_{1}\right)>s\left(\alpha_{2}\right)$, then $\alpha_{1}>\alpha_{2}$.

(2) If $s\left(\alpha_{1}\right)=s\left(\alpha_{2}\right)$, then the following hold.

(a) If $h\left(\alpha_{1}\right)>h\left(\alpha_{2}\right)$, then $\alpha_{1}>\alpha_{2}$.

(b) If $h\left(\alpha_{1}\right)=h\left(\alpha_{2}\right)$, then $\alpha_{1}=\alpha_{2}$.

(c) If $h\left(\alpha_{2}\right)>h\left(\alpha_{1}\right)$, then $\alpha_{2}>\alpha_{1}$.

2.3. Interval-Valued Intuitionistic Hesitant Fuzzy Sets and Interval-Valued Intuitionistic Hesitant Fuzzy Elements. In the following, we propose the concept of interval-valued intuitionistic hesitant fuzzy sets, which permit the membership of an element to be a set of several possible interval-valued intuitionistic fuzzy numbers. The motivation is that when defining the membership degree of an element, the difficulty of establishing the membership degree is not because we have a margin of error (as in intuitionistic fuzzy sets) or some possibility distribution (as in type 2 fuzzy sets) on the possible values, but because we have several possible interval-valued intuitionistic fuzzy numbers.
Definition 6. Let $X$ be a fixed set; an interval-valued intuitionistic hesitant fuzzy set (IVIHFS) on $X$ is given in terms of a function that when applied to $X$ returns a subset of $\Omega$.

To be easily understood, we express the IVIHFS by a mathematical symbol

$$
\widetilde{E}=\left\{\left\langle x, h_{\widetilde{E}}(x)\right\rangle \mid x \in X\right\},
$$

where $h_{\widetilde{E}}(x)$ is a set of some IVIFNs in $\Omega$, denoting the possible membership degree intervals and nonmembership degree intervals of the element $x \in X$ to the set $\widetilde{E}$. For convenience, we call $\widetilde{h}=h_{\widetilde{E}}(x)$ an interval-valued intuitionistic hesitant fuzzy element (IVIHFE) and $\widetilde{H}$ the set of all IVIHFEs. If $\alpha \in \widetilde{h}$, then $\alpha$ is an IVIFN, and it can be denoted by $\alpha=\left(\mu_{\alpha}, v_{\alpha}\right)=\left(\left[\mu_{\alpha}^{-}, \mu_{\alpha}^{+}\right],\left[v_{\alpha}^{-}, \nu_{\alpha}^{+}\right]\right)$.

For any $\alpha \in \widetilde{h}$, if $\alpha$ is a real number in [0,1], then $\tilde{h}$ reduces to a hesitant fuzzy element (HFE) [9]; if $\alpha$ is a closed subinterval of the unit interval, then $\tilde{h}$ reduces to an interval-valued hesitant fuzzy element (IVHFE) [6]; if $\alpha$ is an intuitionistic fuzzy number (IFN) [11], then $\widetilde{h}$ reduces to an intuitionistic hesitant fuzzy element (IHFE). Therefore, HFEs, IVHFEs, and IHFEs are special cases of IVIHFEs.

Definition 7 . Given three IVIHFEs represented by $\widetilde{h}, \widetilde{h}_{1}$, and $\widetilde{h}_{2}$, one defines some operations on them, which can be described as

$$
\begin{aligned}
& \widetilde{h}^{c}=\left\{\alpha^{c} \mid \alpha \in \widetilde{h}\right\}=\left\{\left(\left[\nu_{\alpha}^{-}, \nu_{\alpha}^{+}\right],\left[\mu_{\alpha}^{-}, \mu_{\alpha}^{+}\right]\right) \mid \alpha \in \widetilde{h}\right\}, \\
& \widetilde{h}_{1} \cup \widetilde{h}_{2}=\left\{\alpha_{1} \vee \alpha_{2} \mid \alpha_{1} \in \widetilde{h}_{1}, \alpha_{2} \in \widetilde{h}_{2}\right\} \\
& =\left\{\left(\left[\mu_{\alpha_{1}}^{-} \vee \mu_{\alpha_{2}}^{-}, \mu_{\alpha_{1}}^{+} \vee \mu_{\alpha_{2}}^{+}\right]\right.\right. \text {, } \\
& \left.\left[v_{\alpha_{1}}^{-} \wedge v_{\alpha_{2}}^{-}, v_{\alpha_{1}}^{+} \wedge v_{\alpha_{2}}^{+}\right]\right) \mid \\
& \left.\alpha_{1} \in \widetilde{h}_{1}, \alpha_{2} \in \widetilde{h}_{2}\right\}, \\
& \widetilde{h}_{1} \cap \widetilde{h}_{2}=\left\{\alpha_{1} \wedge \alpha_{2} \mid \alpha_{1} \in \widetilde{h}_{1}, \alpha_{2} \in \widetilde{h}_{2}\right\} \\
& =\left\{\left(\left[\mu_{\alpha_{1}}^{-} \wedge \mu_{\alpha_{2}}^{-}, \mu_{\alpha_{1}}^{+} \wedge \mu_{\alpha_{2}}^{+}\right],\right.\right. \\
& \left.\left[v_{\alpha_{1}}^{-} \vee v_{\alpha_{2}}^{-}, v_{\alpha_{1}}^{+} \vee v_{\alpha_{2}}^{+}\right]\right) \text {| } \\
& \left.\alpha_{1} \in \widetilde{h}_{1}, \alpha_{2} \in \tilde{h}_{2}\right\} \text {, } \\
& \widetilde{h}_{1} \otimes \widetilde{h}_{2}=\left\{\alpha_{1} \otimes \alpha_{2} \mid \alpha_{1} \in \widetilde{h}_{1}, \alpha_{2} \in \widetilde{h}_{2}\right\} \\
& =\left\{\left(\left[\mu_{\alpha_{1}}^{-} \mu_{\alpha_{2}}^{-}, \mu_{\alpha_{1}}^{+} \mu_{\alpha_{2}}^{+}\right]\right.\right. \text {, } \\
& \left.\left[v_{\alpha_{1}}^{-}+v_{\alpha_{2}}^{-}-v_{\alpha_{1}}^{-} v_{\alpha_{2}}^{-}, v_{\alpha_{1}}^{+}+v_{\alpha_{2}}^{+}-v_{\alpha_{1}}^{+} v_{\alpha_{2}}^{+}\right]\right) \mid \\
& \left.\alpha_{1} \in \widetilde{h}_{1}, \alpha_{2} \in \widetilde{h}_{2}\right\} \text {, } \\
& \widetilde{h}_{1} \oplus \widetilde{h}_{2}=\left\{\alpha_{1} \oplus \alpha_{2} \mid \alpha_{1} \in \widetilde{h}_{1}, \alpha_{2} \in \widetilde{h}_{2}\right\} \\
& =\left\{\left(\left[\mu_{\alpha_{1}}^{-}+\mu_{\alpha_{2}}^{-}-\mu_{\alpha_{1}}^{-} \mu_{\alpha_{2}}^{-}, \mu_{\alpha_{1}}^{+}+\mu_{\alpha_{2}}^{+}-\mu_{\alpha_{1}}^{+} \mu_{\alpha_{2}}^{+}\right],\right.\right. \\
& \left.\left.\left[v_{\alpha_{1}}^{-} v_{\alpha_{2}}^{-}, v_{\alpha_{1}}^{+} v_{\alpha_{2}}^{+}\right]\right) \mid \alpha_{1} \in \widetilde{h}_{1}, \alpha_{2} \in \widetilde{h}_{2}\right\} \text {, }
\end{aligned}
$$




$$
\begin{aligned}
& \lambda \widetilde{h}=\{\lambda \alpha \mid \alpha \in \widetilde{h}\} \\
&=\left\{\left(\left[1-\left(1-\mu_{\alpha}^{-}\right)^{\lambda}, 1-\left(1-\mu_{\alpha}^{+}\right)^{\lambda}\right],\right.\right. \\
& {\left.\left.\left[\left(\nu_{\alpha}^{-}\right)^{\lambda},\left(\nu_{\alpha}^{+}\right)^{\lambda}\right]\right) \mid \alpha \in \widetilde{h}\right\}, \quad \lambda>0, } \\
& \widetilde{h}^{\lambda}=\left\{\alpha^{\lambda} \mid \alpha \in \widetilde{h}\right\}\left\{\left(\left[\left(\mu_{\alpha}^{-}\right)^{\lambda},\left(\mu_{\alpha}^{+}\right)^{\lambda}\right],\right.\right. \\
& {\left.\left.\left[1-\left(1-v_{\alpha}^{-}\right)^{\lambda}, 1-\left(1-v_{\alpha}^{+}\right)^{\lambda}\right]\right) \mid \alpha \in \widetilde{h}\right\}, } \\
& \lambda>0 .
\end{aligned}
$$

Theorem 8. Let $\widetilde{h}, \widetilde{h}_{1}$, and $\widetilde{h}_{2}$ be three IVIHFEs, and $\lambda \geq 0$. Then, $\widetilde{h}_{1} \oplus \widetilde{h}_{2}, \widetilde{h}_{1} \otimes \widetilde{h}_{2}, \lambda \widetilde{h}$, and $\widetilde{h}^{\lambda}$ are also IVIHFEs.

Proof. Since $\widetilde{h}=\left\{\left(\left[\mu_{\alpha}^{-}, \mu_{\alpha}^{+}\right],\left[v_{\alpha}^{-}, v_{\alpha}^{+}\right]\right) \quad \mid \alpha \in \tilde{h}\right\}$, $\widetilde{h}_{1}=\left\{\left(\left[\mu_{\alpha_{1}}^{-}, \mu_{\alpha_{1}}^{+}\right],\left[\nu_{\alpha_{1}}^{-}, v_{\alpha_{1}}^{+}\right]\right) \mid \alpha_{1} \in \widetilde{h}_{1}\right\}$, and $\widetilde{h}_{2}=$ $\left\{\left(\left[\mu_{\alpha_{2}}^{-}, \mu_{\alpha_{2}}^{+}\right],\left[v_{\alpha_{2}}^{-}, v_{\alpha_{2}}^{+}\right]\right) \mid \alpha_{2} \in \widetilde{h}_{2}\right\}$ are three IVIHFEs, we have $0 \leq \mu_{\alpha}^{-}, \mu_{\alpha}^{+}, v_{\alpha}^{-}, v_{\alpha}^{+}, \mu_{\alpha_{1}}^{-}, \mu_{\alpha_{1}}^{+}, v_{\alpha_{1}}^{-}, v_{\alpha_{1}}^{+}, \mu_{\alpha_{2}}^{-}, \mu_{\alpha_{2}}^{+}, v_{\alpha_{2}}^{-}, v_{\alpha_{2}}^{+} \leq 1$, $\mu_{\alpha}^{+}+\nu_{\alpha}^{+} \leq 1, \mu_{\alpha_{1}}^{+}+\nu_{\alpha_{1}}^{+} \leq 1$, and $\mu_{\alpha_{2}}^{+}+\nu_{\alpha_{2}}^{+} \leq 1$. Then, we can obtain

$$
\begin{aligned}
& 0 \leq \mu_{\alpha_{1}}^{-}+\mu_{\alpha_{2}}^{-}-\mu_{\alpha_{1}}^{-} \mu_{\alpha_{2}}^{-}=1-\left(1-\mu_{\alpha_{1}}^{-}\right)\left(1-\mu_{\alpha_{2}}^{-}\right) \\
& \leq 1-\left(1-\mu_{\alpha_{1}}^{+}\right)\left(1-\mu_{\alpha_{2}}^{+}\right)=\mu_{\alpha_{1}}^{+}+\mu_{\alpha_{2}}^{+}-\mu_{\alpha_{1}}^{+} \mu_{\alpha_{2}}^{+} \leq 1 \text {, } \\
& 0 \leq v_{\alpha_{1}}^{-} v_{\alpha_{2}}^{-} \leq v_{\alpha_{1}}^{+} v_{\alpha_{2}}^{+} \leq 1, \\
& \mu_{\alpha_{1}}^{+}+\mu_{\alpha_{2}}^{+}-\mu_{\alpha_{1}}^{+} \mu_{\alpha_{2}}^{+}+v_{\alpha_{1}}^{+} v_{\alpha_{2}}^{+} \\
& =1-\left(1-\mu_{\alpha_{1}}^{+}\right)\left(1-\mu_{\alpha_{2}}^{+}\right)+v_{\alpha_{1}}^{+} v_{\alpha_{2}}^{+} \\
& \leq 1-\left(1-\mu_{\alpha_{1}}^{+}\right)\left(1-\mu_{\alpha_{2}}^{+}\right) \\
& +\left(1-\mu_{\alpha_{1}}^{+}\right)\left(1-\mu_{\alpha_{2}}^{+}\right)=1 \text {, } \\
& 0 \leq \mu_{\alpha_{1}}^{-} \mu_{\alpha_{2}}^{-} \leq \mu_{\alpha_{1}}^{+} \mu_{\alpha_{2}}^{+} \leq 1 \text {, } \\
& 0 \leq v_{\alpha_{1}}^{-}+v_{\alpha_{2}}^{-}-v_{\alpha_{1}}^{-} v_{\alpha_{2}}^{-}=1-\left(1-v_{\alpha_{1}}^{-}\right)\left(1-v_{\alpha_{2}}^{-}\right) \\
& \leq 1-\left(1-v_{\alpha_{1}}^{+}\right)\left(1-v_{\alpha_{2}}^{+}\right)=v_{\alpha_{1}}^{+}+v_{\alpha_{2}}^{+}-v_{\alpha_{1}}^{+} v_{\alpha_{2}}^{+} \leq 1 \text {, } \\
& \mu_{\alpha_{1}}^{+} \mu_{\alpha_{2}}^{+}+v_{\alpha_{1}}^{+}+v_{\alpha_{2}}^{+}-v_{\alpha_{1}}^{+} \nu_{\alpha_{2}}^{+} \\
& =\mu_{\alpha_{1}}^{+} \mu_{\alpha_{2}}^{+}+1-\left(1-v_{\alpha_{1}}^{+}\right)\left(1-v_{\alpha_{2}}^{+}\right) \\
& \leq\left(1-v_{\alpha_{1}}^{+}\right)\left(1-v_{\alpha_{2}}^{+}\right) \\
& +1-\left(1-v_{\alpha_{1}}^{+}\right)\left(1-v_{\alpha_{2}}^{+}\right)=1, \\
& 0 \leq 1-\left(1-\mu_{\alpha}^{-}\right)^{\lambda} \leq 1-\left(1-\mu_{\alpha}^{+}\right)^{\lambda} \leq 1,
\end{aligned}
$$

$$
\begin{gathered}
0 \leq\left(v_{\alpha}^{-}\right)^{\lambda} \leq\left(v_{\alpha}^{+}\right)^{\lambda} \leq 1, \\
1-\left(1-\mu_{\alpha}^{+}\right)^{\lambda}+\left(v_{\alpha}^{+}\right)^{\lambda} \\
\leq 1-\left(1-\mu_{\alpha}^{+}\right)^{\lambda}+\left(1-\mu_{\alpha}^{+}\right)^{\lambda}=1, \\
0 \leq\left(\mu_{\alpha}^{-}\right)^{\lambda} \leq\left(\mu_{\alpha}^{+}\right)^{\lambda} \leq 1, \\
0 \leq 1-\left(1-v_{\alpha}^{-}\right)^{\lambda} \leq 1-\left(1-v_{\alpha}^{+}\right)^{\lambda} \leq 1, \\
\left(\mu_{\alpha}^{+}\right)^{\lambda}+1-\left(1-v_{\alpha}^{+}\right)^{\lambda} \leq\left(1-v_{\alpha}^{+}\right)^{\lambda}+1-\left(1-v_{\alpha}^{+}\right)^{\lambda}=1 .
\end{gathered}
$$

Thus, $\widetilde{h}_{1} \oplus \widetilde{h}_{2}, \widetilde{h}_{1} \otimes \widetilde{h}_{2}, \lambda \widetilde{h}$, and $\widetilde{h}^{\lambda}$ are IVIHFEs.

Theorem 9. Let $\widetilde{h}, \widetilde{h}_{1}$, and $\widetilde{h}_{2}$ be three IVIHFEs, and $\lambda$, $\lambda_{1}$, and $\lambda_{2}>0$. Then, one has

$$
\begin{gathered}
\widetilde{h}_{1}^{c} \cup \widetilde{h}_{2}^{c}=\left(\widetilde{h}_{1} \cap \widetilde{h}_{2}\right)^{c}, \\
\widetilde{h}_{1}^{c} \cap \widetilde{h}_{2}^{c}=\left(\widetilde{h}_{1} \cup \widetilde{h}_{2}\right)^{c}, \\
\left(\widetilde{h}^{c}\right)^{\lambda}=(\lambda \widetilde{h})^{c}, \\
\lambda\left(\widetilde{h}^{c}\right)=\left(\widetilde{h}^{\lambda}\right)^{c}, \\
\widetilde{h}_{1}^{c} \oplus \widetilde{h}_{2}^{c}=\left(\widetilde{h}_{1} \otimes \widetilde{h}_{2}\right)^{c}, \\
\widetilde{h}_{1}^{c} \otimes \widetilde{h}_{2}^{c}=\left(\widetilde{h}_{1} \oplus \widetilde{h}_{2}\right)^{c}, \\
\widetilde{h}_{1} \oplus \widetilde{h}_{2}=\widetilde{h}_{2} \oplus \widetilde{h}_{1}, \\
\lambda\left(\widetilde{h}_{1} \oplus \widetilde{h}_{2}\right)=\lambda \widetilde{h}_{1} \oplus \lambda \widetilde{h}_{2}, \\
\left(\lambda_{1} \lambda_{2}\right) \widetilde{h}=\lambda_{1}\left(\lambda_{2} \widetilde{h}^{2},\right. \\
\widetilde{h}_{1} \otimes \widetilde{h}_{2}=\widetilde{h}_{2} \otimes \widetilde{h}_{1}, \\
\widetilde{h}_{1}^{\lambda} \otimes \widetilde{h}_{2}^{\lambda}=\left(\widetilde{h}_{1} \otimes \widetilde{h}_{2}\right)^{\lambda}, \\
\widetilde{h}^{\lambda_{1} \lambda_{2}}=\left(\widetilde{h}^{\lambda_{1}}\right)^{\lambda_{2}} .
\end{gathered}
$$

Proof. Consider

$$
\begin{aligned}
& \tilde{h}_{1}^{c} \cup \widetilde{h}_{2}^{c} \\
&=\left\{\left(\left[\nu_{\alpha_{1}}^{-}, v_{\alpha_{1}}^{+}\right],\left[\mu_{\alpha_{1}}^{-}, \mu_{\alpha_{1}}^{+}\right]\right) \mid \alpha_{1} \in \widetilde{h}_{1}\right\} \\
& \cup\left\{\left(\left[\nu_{\alpha_{2}}^{-}, v_{\alpha_{2}}^{+}\right],\left[\mu_{\alpha_{2}}^{-}, \mu_{\alpha_{2}}^{+}\right]\right) \mid \alpha_{2} \in \widetilde{h}_{2}\right\} \\
&=\left\{\left(\left[\nu_{\alpha_{1}}^{-} \vee v_{\alpha_{2}}^{-}, v_{\alpha_{1}}^{+} \vee v_{\alpha_{2}}^{+}\right],\left[\mu_{\alpha_{1}}^{-} \wedge \mu_{\alpha_{2}}^{-}, \mu_{\alpha_{1}}^{+} \wedge \mu_{\alpha_{2}}^{+}\right]\right) \mid\right. \\
&\left.\alpha_{1} \in \widetilde{h}_{1}, \alpha_{2} \in \widetilde{h}_{2}\right\} \\
&=\left\{\left(\left[\mu_{\alpha_{1}}^{-} \wedge \mu_{\alpha_{2}}^{-}, \mu_{\alpha_{1}}^{+} \wedge \mu_{\alpha_{2}}^{+}\right],\left[\nu_{\alpha_{1}}^{-} \vee v_{\alpha_{2}}^{-}, v_{\alpha_{1}}^{+} \vee v_{\alpha_{2}}^{+}\right]\right) \mid\right. \\
&\left.\alpha_{1} \in \widetilde{h}_{1}, \alpha_{2} \in \widetilde{h}_{2}\right\}^{c}=\left(\widetilde{h}_{1} \cap \widetilde{h}_{2}\right)^{c},
\end{aligned}
$$


Journal of Applied Mathematics

5

$$
\begin{aligned}
\widetilde{h}_{1}^{c} \cap \widetilde{h}_{2}^{c} & \left\{\left(\left[v_{\alpha_{1}}^{-}, v_{\alpha_{1}}^{+}\right],\left[\mu_{\alpha_{1}}^{-}, \mu_{\alpha_{1}}^{+}\right]\right) \mid \alpha_{1} \in \widetilde{h}_{1}\right\} \\
& \cap\left\{\left(\left[v_{\alpha_{2}}^{-}, v_{\alpha_{2}}^{+}\right],\left[\mu_{\alpha_{2}}^{-}, \mu_{\alpha_{2}}^{+}\right]\right) \mid \alpha_{2} \in \widetilde{h}_{2}\right\} \\
= & \left\{\left(\left[v_{\alpha_{1}}^{-} \wedge v_{\alpha_{2}}^{-}, v_{\alpha_{1}}^{+} \wedge v_{\alpha_{2}}^{+}\right],\left[\mu_{\alpha_{1}}^{-} \vee \mu_{\alpha_{2}}^{-}, \mu_{\alpha_{1}}^{+} \vee \mu_{\alpha_{2}}^{+}\right]\right) \mid\right. \\
& \left.\alpha_{1} \in \widetilde{h}_{1}, \alpha_{2} \in \widetilde{h}_{2}\right\} \\
= & \left\{\left(\left[\mu_{\alpha_{1}}^{-} \vee \mu_{\alpha_{2}}^{-}, \mu_{\alpha_{1}}^{+} \vee \mu_{\alpha_{2}}^{+}\right],\left[\nu_{\alpha_{1}}^{-} \wedge v_{\alpha_{2}}^{-}, v_{\alpha_{1}}^{+} \wedge v_{\alpha_{2}}^{+}\right]\right) \mid\right. \\
& \left.\alpha_{1} \in \widetilde{h}_{1}, \alpha_{2} \in \widetilde{h}_{2}\right\}^{c}=\left(\widetilde{h}_{1} \cup \widetilde{h}_{2}\right)^{c}, \\
\left(\widetilde{h}^{c}\right)^{\lambda}=\{ & \left\{\left(\left[v_{\alpha}^{-}, v_{\alpha}^{+}\right],\left[\mu_{\alpha}^{-}, \mu_{\alpha}^{+}\right]\right) \mid \alpha \in \widetilde{h}\right\}^{\lambda} \\
=\{ & \left\{\left(\left[\left(v_{\alpha}^{-}\right)^{\lambda},\left(v_{\alpha}^{+}\right)^{\lambda}\right],\left[1-\left(1-\mu_{\alpha}^{-}\right)^{\lambda}, 1-\left(1-\mu_{\alpha}^{+}\right)^{\lambda}\right]\right) \mid\right. \\
& \alpha \in \widetilde{h}\}=(\lambda \widetilde{h})^{c}, \\
\lambda\left(\widetilde{h}^{c}\right)= & \lambda\left\{\left(\left[v_{\alpha}^{-}, v_{\alpha}^{+}\right],\left[\mu_{\alpha}^{-}, \mu_{\alpha}^{+}\right]\right) \mid \alpha \in \widetilde{h}\right\} \\
=\{ & \left\{\left(\left[1-\left(1-v_{\alpha}^{-}\right)^{\lambda}, 1-\left(1-v_{\alpha}^{+}\right)^{\lambda}\right],\right.\right. \\
& {\left.\left.\left[\left(\mu_{\alpha}^{-}\right)^{\lambda},\left(\mu_{\alpha}^{+}\right)^{\lambda}\right]\right) \mid \alpha \in \widetilde{h}\right\}=\left(\widetilde{h}^{\lambda}\right)^{c}, }
\end{aligned}
$$

$\widetilde{h}_{1}^{c} \oplus \widetilde{h}_{2}^{c}$

$$
\begin{aligned}
&=\left\{\left(\left[v_{\alpha_{1}}^{-}, v_{\alpha_{1}}^{+}\right],\left[\mu_{\alpha_{1}}^{-}, \mu_{\alpha_{1}}^{+}\right]\right) \mid \alpha_{1} \in \widetilde{h}_{1}\right\} \\
& \oplus\left\{\left(\left[\nu_{\alpha_{2}}^{-}, v_{\alpha_{2}}^{+}\right],\left[\mu_{\alpha_{2}}^{-}, \mu_{\alpha_{2}}^{+}\right]\right) \mid \alpha_{2} \in \widetilde{h}_{2}\right\} \\
&=\left\{\left(\left[\nu_{\alpha_{1}}^{-}+v_{\alpha_{2}}^{-}-v_{\alpha_{1}}^{-} v_{\alpha_{2}}^{-}, v_{\alpha_{1}}^{+}+v_{\alpha_{2}}^{+}-v_{\alpha_{1}}^{+} v_{\alpha_{2}}^{+}\right],\right.\right. \\
& {\left.\left.\left[\mu_{\alpha_{1}}^{-} \mu_{\alpha_{2}}^{-}, \mu_{\alpha_{1}}^{+} \mu_{\alpha_{2}}^{+}\right]\right) \mid \alpha_{1} \in \widetilde{h}_{1}, \alpha_{2} \in \widetilde{h}_{2}\right\} } \\
&=\left\{\left(\left[\mu_{\alpha_{1}}^{-} \mu_{\alpha_{2}}^{-}, \mu_{\alpha_{1}}^{+} \mu_{\alpha_{2}}^{+}\right],\right.\right. \\
& {\left.\left[v_{\alpha_{1}}^{-}+v_{\alpha_{2}}^{-}-v_{\alpha_{1}}^{-} v_{\alpha_{2}}^{-}, v_{\alpha_{1}}^{+}+v_{\alpha_{2}}^{+}-v_{\alpha_{1}}^{+} \nu_{\alpha_{2}}^{+}\right]\right) \mid } \\
&\left.\alpha_{1} \in \widetilde{h}_{1}, \alpha_{2} \in \widetilde{h}_{2}\right\}^{c}=\left(\widetilde{h}_{1} \otimes \widetilde{h}_{2}\right)^{c},
\end{aligned}
$$

$\widetilde{h}_{1}^{c} \oplus \widetilde{h}_{2}^{c}$

$$
\begin{aligned}
= & \left\{\left(\left[v_{\alpha_{1}}^{-}, v_{\alpha_{1}}^{+}\right],\left[\mu_{\alpha_{1}}^{-}, \mu_{\alpha_{1}}^{+}\right]\right) \mid \alpha_{1} \in \widetilde{h}_{1}\right\} \\
\otimes & \left\{\left(\left[\nu_{\alpha_{2}}^{-}, v_{\alpha_{2}}^{+}\right],\left[\mu_{\alpha_{2}}^{-}, \mu_{\alpha_{2}}^{+}\right]\right) \mid \alpha_{2} \in \widetilde{h}_{2}\right\} \\
= & \left\{\left(\left[\nu_{\alpha_{1}}^{-} v_{\alpha_{2}}^{-}, v_{\alpha_{1}}^{+} v_{\alpha_{2}}^{+}\right],\right.\right. \\
& {\left.\left[\mu_{\alpha_{1}}^{-}+\mu_{\alpha_{2}}^{-}-\mu_{\alpha_{1}}^{-} \mu_{\alpha_{2}}^{-}, \mu_{\alpha_{1}}^{+}+\mu_{\alpha_{2}}^{+}-\mu_{\alpha_{1}}^{+} \mu_{\alpha_{2}}^{+}\right]\right) \mid } \\
& \left.\alpha_{1} \in \widetilde{h}_{1}, \alpha_{2} \in \widetilde{h}_{2}\right\} \\
= & \left\{\left(\left[\mu_{\alpha_{1}}^{-}+\mu_{\alpha_{2}}^{-}-\mu_{\alpha_{1}}^{-} \mu_{\alpha_{2}}^{-}, \mu_{\alpha_{1}}^{+}+\mu_{\alpha_{2}}^{+}-\mu_{\alpha_{1}}^{+} \mu_{\alpha_{2}}^{+}\right],\right.\right. \\
& {\left.\left.\left[\nu_{\alpha_{1}}^{-} v_{\alpha_{2}}^{-}, v_{\alpha_{1}}^{+} v_{\alpha_{2}}^{+}\right]\right) \mid \alpha_{1} \in \widetilde{h}_{1}, \alpha_{2} \in \widetilde{h}_{2}\right\}^{c} } \\
= & \left(\widetilde{h}_{1} \oplus \widetilde{h}_{2}\right)^{c},
\end{aligned}
$$

$\widetilde{h}_{1} \oplus \widetilde{h}_{2}$

$$
\begin{gathered}
=\left\{\left(\left[\mu_{\alpha_{1}}^{-}+\mu_{\alpha_{2}}^{-}-\mu_{\alpha_{1}}^{-} \mu_{\alpha_{2}}^{-}, \mu_{\alpha_{1}}^{+}+\mu_{\alpha_{2}}^{+}-\mu_{\alpha_{1}}^{+} \mu_{\alpha_{2}}^{+}\right],\right.\right. \\
\left.\left.\left[v_{\alpha_{1}}^{-} v_{\alpha_{2}}^{-}, v_{\alpha_{1}}^{+} v_{\alpha_{2}}^{+}\right]\right) \mid \alpha_{1} \in \widetilde{h}_{1}, \alpha_{2} \in \widetilde{h}_{2}\right\} \\
=\left\{\left(\left[\mu_{\alpha_{2}}^{-}+\mu_{\alpha_{1}}^{-}-\mu_{\alpha_{2}}^{-} \mu_{\alpha_{1}}^{-}, \mu_{\alpha_{2}}^{+}+\mu_{\alpha_{1}}^{+}-\mu_{\alpha_{2}}^{+} \mu_{\alpha_{1}}^{+}\right],\right.\right. \\
\left.\left.\left[\nu_{\alpha_{2}}^{-} v_{\alpha_{1}}^{-}, v_{\alpha_{2}}^{+} v_{\alpha_{1}}^{+}\right]\right) \mid \alpha_{2} \in \widetilde{h}_{2}, \alpha_{1} \in \widetilde{h}_{1}\right\} \\
=\widetilde{h}_{2} \oplus \widetilde{h}_{1},
\end{gathered}
$$

$$
\lambda\left(\widetilde{h}_{1} \oplus \widetilde{h}_{2}\right)
$$$$
=\lambda\left\{\left(\left[\mu_{\alpha_{1}}^{-}+\mu_{\alpha_{2}}^{-}-\mu_{\alpha_{1}}^{-} \mu_{\alpha_{2}}^{-}, \mu_{\alpha_{1}}^{+}+\mu_{\alpha_{2}}^{+}-\mu_{\alpha_{1}}^{+} \mu_{\alpha_{2}}^{+}\right],\right.\right.
$$$$
\left.\left.\left[v_{\alpha_{1}}^{-} v_{\alpha_{2}}^{-}, v_{\alpha_{1}}^{+} v_{\alpha_{2}}^{+}\right]\right) \mid \alpha_{1} \in \widetilde{h}_{1}, \alpha_{2} \in \widetilde{h}_{2}\right\}
$$

$$
=\left\{\left(\left[1-\left(1-\mu_{\alpha_{1}}^{-}-\mu_{\alpha_{2}}^{-}+\mu_{\alpha_{1}}^{-} \mu_{\alpha_{2}}^{-}\right)^{\lambda}\right.\right.\right. \text {, }
$$$$
\left.1-\left(1-\mu_{\alpha_{1}}^{+}-\mu_{\alpha_{2}}^{+}+\mu_{\alpha_{1}}^{+} \mu_{\alpha_{2}}^{+}\right)^{\lambda}\right],
$$$$
\left.\left[\left(v_{\alpha_{1}}^{-} v_{\alpha_{2}}^{-}\right)^{\lambda},\left(v_{\alpha_{1}}^{+} v_{\alpha_{2}}^{+}\right)^{\lambda}\right]\right) \text { | }
$$$$
\left.\alpha_{1} \in \widetilde{h}_{1}, \alpha_{2} \in \widetilde{h}_{2}\right\}
$$

$$
=\left\{\left(\left[1-\left(1-\mu_{\alpha_{1}}^{-}\right)^{\lambda}\left(1-\mu_{\alpha_{2}}^{-}\right)^{\lambda}\right.\right.\right. \text {, }
$$$$
\left.1-\left(1-\mu_{\alpha_{1}}^{+}\right)^{\lambda}\left(1-\mu_{\alpha_{2}}^{+}\right)^{\lambda}\right],
$$$$
\left.\left[\left(v_{\alpha_{1}}^{-}\right)^{\lambda}\left(v_{\alpha_{2}}^{-}\right)^{\lambda},\left(v_{\alpha_{1}}^{+}\right)^{\lambda}\left(v_{\alpha_{2}}^{+}\right)^{\lambda}\right]\right) \mid
$$$$
\left.\alpha_{1} \in \widetilde{h}_{1}, \alpha_{2} \in \widetilde{h}_{2}\right\},
$$

$$
\begin{gathered}
\lambda \widetilde{h}_{1} \oplus \lambda \widetilde{h}_{2} \\
=\left\{\left(\left[1-\left(1-\mu_{\alpha_{1}}^{-}\right)^{\lambda}, 1-\left(1-\mu_{\alpha_{1}}^{+}\right)^{\lambda}\right],\right.\right. \\
\left.\left.\left[\left(v_{\alpha_{1}}^{-}\right)^{\lambda},\left(v_{\alpha_{1}}^{+}\right)^{\lambda}\right]\right) \mid \alpha_{1} \in \widetilde{h}_{1}\right\} \\
\oplus\left\{\left(\left[1-\left(1-\mu_{\alpha_{2}}^{-}\right)^{\lambda}, 1-\left(1-\mu_{\alpha_{2}}^{+}\right)^{\lambda}\right],\right.\right. \\
\left.\left.\left[\left(v_{\alpha_{2}}^{-}\right)^{\lambda},\left(v_{\alpha_{2}}^{+}\right)^{\lambda}\right]\right) \mid \alpha_{2} \in \widetilde{h}_{2}\right\} \\
=\left\{\left(\left[1-\left(1-\mu_{\alpha_{1}}^{-}\right)^{\lambda}+1-\left(1-\mu_{\alpha_{2}}^{-}\right)^{\lambda}\right.\right.\right. \\
\quad-\left(1-\left(1-\mu_{\alpha_{1}}^{-}\right)^{\lambda}\right)\left(1-\left(1-\mu_{\alpha_{2}}^{-}\right)^{\lambda}\right), \\
1-\left(1-\mu_{\alpha_{1}}^{+}\right)^{\lambda}+1-\left(1-\mu_{\alpha_{2}}^{+}\right)^{\lambda} \\
\left.-\left(1-\left(1-\mu_{\alpha_{1}}^{+}\right)^{\lambda}\right)\left(1-\left(1-\mu_{\alpha_{2}}^{+}\right)^{\lambda}\right)\right],
\end{gathered}
$$


6

Journal of Applied Mathematics

$$
\begin{gathered}
\left.\left[\left(v_{\alpha_{1}}^{-}\right)^{\lambda}\left(v_{\alpha_{2}}^{-}\right)^{\lambda},\left(v_{\alpha_{1}}^{+}\right)^{\lambda}\left(v_{\alpha_{2}}^{+}\right)^{\lambda}\right]\right) \mid \\
\left.\alpha_{1} \in \widetilde{h}_{1}, \alpha_{2} \in \widetilde{h}_{2}\right\} \\
=\left\{\left(\left[1-\left(1-\mu_{\alpha_{1}}^{-}\right)^{\lambda}\left(1-\mu_{\alpha_{2}}^{-}\right)^{\lambda},\right.\right.\right. \\
\left.1-\left(1-\mu_{\alpha_{1}}^{+}\right)^{\lambda}\left(1-\mu_{\alpha_{2}}^{+}\right)^{\lambda}\right], \\
\left.\left[\left(v_{\alpha_{1}}^{-}\right)^{\lambda}\left(v_{\alpha_{2}}^{-}\right)^{\lambda},\left(v_{\alpha_{1}}^{+}\right)^{\lambda}\left(v_{\alpha_{2}}^{+}\right)^{\lambda}\right]\right) \mid \\
\left.\alpha_{1} \in \widetilde{h}_{1}, \alpha_{2} \in \widetilde{h}_{2}\right\} .
\end{gathered}
$$

(10)

Thus, we have $\lambda\left(\widetilde{h}_{1} \oplus \widetilde{h}_{2}\right)=\lambda \widetilde{h}_{1} \oplus \lambda \widetilde{h}_{2}$.

Moreover,

$$
\begin{gathered}
\left(\lambda_{1} \lambda_{2}\right) \tilde{h}=\left\{\left(\left[1-\left(1-\mu_{\alpha}^{-}\right)^{\lambda_{1} \lambda_{2}}, 1-\left(1-\mu_{\alpha}^{+}\right)^{\lambda_{1} \lambda_{2}}\right],\right.\right. \\
\left.\left.\quad\left[\left(v_{\alpha}^{-}\right)^{\lambda_{1} \lambda_{2}},\left(v_{\alpha}^{+}\right)^{\lambda_{1} \lambda_{2}}\right]\right) \mid \alpha \in \widetilde{h}\right\}, \\
\lambda_{1}\left(\lambda_{2} \widetilde{h}\right) \\
=\lambda_{1}\left(\left\{\left(\left[1-\left(1-\mu_{\alpha}^{-}\right)^{\lambda_{2}}, 1-\left(1-\mu_{\alpha}^{+}\right)^{\lambda_{2}}\right],\right.\right.\right. \\
\left.\left.\left.\left[\left(v_{\alpha}^{-}\right)^{\lambda_{2}},\left(v_{\alpha}^{+}\right)^{\lambda_{2}}\right]\right) \mid \alpha \in \widetilde{h}\right\}\right) \\
=\left\{\left(\left[1-\left(1-1+\left(1-\mu_{\alpha}^{-}\right)^{\lambda_{2}}\right)^{\lambda_{1}},\right.\right.\right. \\
\left.11-\left(1-1+\left(1-\mu_{\alpha}^{+}\right)^{\lambda_{2}}\right)^{\lambda_{1}}\right], \\
\left.\left.\left[\left(\left(v_{\alpha}^{-}\right)^{\lambda_{2}}\right)^{\lambda_{1}},\left(\left(v_{\alpha}^{+}\right)^{\lambda_{2}}\right)^{\lambda_{1}}\right]\right) \mid \alpha \in \tilde{h}\right\} \\
=\left\{\left(\left[1-\left(1-\mu_{\alpha}^{-}\right)^{\lambda_{1} \lambda_{2}}, 1-\left(1-\mu_{\alpha}^{+}\right)^{\lambda_{1} \lambda_{2}}\right],\right.\right. \\
\left.\left.\left[\left(v_{\alpha}^{-}\right)^{\lambda_{1} \lambda_{2}},\left(v_{\alpha}^{+}\right)^{\lambda_{1} \lambda_{2}}\right]\right) \mid \alpha \in \widetilde{h}\right\} .
\end{gathered}
$$

Thus, we have that $\left(\lambda_{1} \lambda_{2}\right) \widetilde{h}=\lambda_{1}\left(\lambda_{2} \widetilde{h}\right)$.

Take

$$
\begin{aligned}
\tilde{h}_{1} \otimes \widetilde{h}_{2} & \\
= & \left\{\left(\left[\mu_{\alpha_{1}}^{-} \mu_{\alpha_{2}}^{-}, \mu_{\alpha_{1}}^{+} \mu_{\alpha_{2}}^{+}\right],\right.\right. \\
& {\left.\left[v_{\alpha_{1}}^{-}+v_{\alpha_{2}}^{-}-v_{\alpha_{1}}^{-} v_{\alpha_{2}}^{-}, v_{\alpha_{1}}^{+}+v_{\alpha_{2}}^{+}-v_{\alpha_{1}}^{+} v_{\alpha_{2}}^{+}\right]\right) \mid } \\
& \left.\alpha_{1} \in \widetilde{h}_{1}, \alpha_{2} \in \widetilde{h}_{2}\right\} \\
= & \left\{\left(\left[\mu_{\alpha_{2}}^{-} \mu_{\alpha_{1}}^{-}, \mu_{\alpha_{2}}^{+} \mu_{\alpha_{1}}^{+}\right],\right.\right. \\
& {\left.\left[v_{\alpha_{2}}^{-}+v_{\alpha_{1}}^{-}-v_{\alpha_{2}}^{-} v_{\alpha_{1}}^{-}, v_{\alpha_{2}}^{+}+v_{\alpha_{1}}^{+}-v_{\alpha_{2}}^{+} v_{\alpha_{1}}^{+}\right]\right) \mid } \\
& \left.\alpha_{2} \in \widetilde{h}_{2}, \alpha_{1} \in \widetilde{h}_{1}\right\}=\widetilde{h}_{2} \otimes \widetilde{h}_{1},
\end{aligned}
$$

$\widetilde{h}_{1}^{\lambda} \otimes \widetilde{h}_{2}^{\lambda}$

$$
\begin{aligned}
& =\left\{\left(\left[\left(\mu_{\alpha_{1}}^{-}\right)^{\lambda},\left(\mu_{\alpha_{1}}^{+}\right)^{\lambda}\right],\right.\right. \\
& \left.\left[1-\left(1-v_{\alpha_{1}}^{-}\right)^{\lambda}, 1-\left(1-v_{\alpha_{1}}^{+}\right)^{\lambda}\right]\right) \mid \\
& \left.\alpha_{1} \in \widetilde{h}_{1}\right\} \\
& \otimes\left\{\left(\left[\left(\mu_{\alpha_{2}}^{-}\right)^{\lambda},\left(\mu_{\alpha_{2}}^{+}\right)^{\lambda}\right],\right.\right. \\
& \left.\left[1-\left(1-v_{\alpha_{2}}^{-}\right)^{\lambda}, 1-\left(1-v_{\alpha_{2}}^{+}\right)^{\lambda}\right]\right) \text { | } \\
& \left.\alpha_{2} \in \tilde{h}_{2}\right\} \\
& =\left\{\left(\left[\left(\mu_{\alpha_{1}}^{-}\right)^{\lambda}\left(\mu_{\alpha_{2}}^{-}\right)^{\lambda},\left(\mu_{\alpha_{1}}^{+}\right)^{\lambda}\left(\mu_{\alpha_{2}}^{+}\right)^{\lambda}\right]\right.\right. \text {, } \\
& {\left[1-\left(1-v_{\alpha_{1}}^{-}\right)^{\lambda}+1-\left(1-v_{\alpha_{2}}^{-}\right)^{\lambda}\right.} \\
& -\left(1-\left(1-v_{\alpha_{1}}^{-}\right)^{\lambda}\right)\left(1-\left(1-v_{\alpha_{2}}^{-}\right)^{\lambda}\right), \\
& 1-\left(1-v_{\alpha_{1}}^{+}\right)^{\lambda}+1-\left(1-v_{\alpha_{2}}^{+}\right)^{\lambda} \\
& -\left(1-\left(1-v_{\alpha_{1}}^{+}\right)^{\lambda}\right) \\
& \left.\left.\times\left(1-\left(1-v_{\alpha_{2}}^{+}\right)^{\lambda}\right)\right]\right) \text { | } \\
& \left.\alpha_{1} \in \widetilde{h}_{1}, \alpha_{2} \in \widetilde{h}_{2}\right\} \\
& =\left\{\left(\left[\left(\mu_{\alpha_{1}}^{-}\right)^{\lambda}\left(\mu_{\alpha_{2}}^{-}\right)^{\lambda},\left(\mu_{\alpha_{1}}^{+}\right)^{\lambda}\left(\mu_{\alpha_{2}}^{+}\right)^{\lambda}\right],\right.\right. \\
& {\left[1-\left(1-v_{\alpha_{1}}^{-}\right)^{\lambda}\left(1-v_{\alpha_{2}}^{-}\right)^{\lambda},\right.} \\
& \left.\left.1-\left(1-v_{\alpha_{1}}^{+}\right)^{\lambda}\left(1-v_{\alpha_{2}}^{+}\right)^{\lambda}\right]\right) \text { | } \\
& \left.\alpha_{1} \in \widetilde{h}_{1}, \alpha_{2} \in \widetilde{h}_{2}\right\} \text {, }
\end{aligned}
$$

$$
\begin{aligned}
\left(\widetilde{h}_{1} \otimes \widetilde{h}_{2}\right)^{\lambda}=\left(\left\{\left(\left[\mu_{\alpha_{1}}^{-} \mu_{\alpha_{2}}^{-}, \mu_{\alpha_{1}}^{+} \mu_{\alpha_{2}}^{+}\right]\right.\right.\right. \\
{\left[\nu_{\alpha_{1}}^{-}+v_{\alpha_{2}}^{-}-v_{\alpha_{1}}^{-} \nu_{\alpha_{2}}^{-},\right.} \\
\left.\left.v_{\alpha_{1}}^{+}+v_{\alpha_{2}}^{+}-v_{\alpha_{1}}^{+} \nu_{\alpha_{2}}^{+}\right]\right) \mid \\
\left.\left.\alpha_{1} \in \widetilde{h}_{1}, \alpha_{2} \in \widetilde{h}_{2}\right\}\right)^{\lambda} \\
=\left\{\left(\left[\left(\mu_{\alpha_{1}}^{-} \mu_{\alpha_{2}}^{-}\right)^{\lambda},\left(\mu_{\alpha_{1}}^{+} \mu_{\alpha_{2}}^{+}\right)^{\lambda}\right],\right.\right. \\
\\
{\left[1-\left(1-v_{\alpha_{1}}^{-}-v_{\alpha_{2}}^{-}+v_{\alpha_{1}}^{-} v_{\alpha_{2}}^{-}\right)^{\lambda}\right.}
\end{aligned}
$$




$$
\begin{aligned}
& \left.\left.\quad 1-\left(1-v_{\alpha_{1}}^{+}-v_{\alpha_{2}}^{+}+v_{\alpha_{1}}^{+} \nu_{\alpha_{2}}^{+}\right)^{\lambda}\right]\right) \mid \\
& \alpha \in \widetilde{h}\} \\
& =\left\{\left(\left[\left(\mu_{\alpha_{1}}^{-} \mu_{\alpha_{2}}^{-}\right)^{\lambda},\left(\mu_{\alpha_{1}}^{+} \mu_{\alpha_{2}}^{+}\right)^{\lambda}\right],\right.\right. \\
& {\left[1-\left(1-v_{\alpha_{1}}^{-}\right)^{\lambda}\left(1-v_{\alpha_{2}}^{-}\right)^{\lambda},\right.} \\
& \left.\left.1-\left(1-v_{\alpha_{1}}^{+}\right)^{\lambda}\left(1-v_{\alpha_{2}}^{+}\right)^{\lambda}\right]\right) \mid \\
& \alpha \in \tilde{h}\} .
\end{aligned}
$$

Thus, we have that $\widetilde{h}_{1}^{\lambda} \otimes \widetilde{h}_{2}^{\lambda}=\left(\widetilde{h}_{1} \otimes \widetilde{h}_{2}\right)^{\lambda}$.

Consider

$$
\begin{aligned}
& \tilde{h}^{\lambda_{1} \lambda_{2}}=\{\left(\left[\left(\mu_{\alpha}^{-}\right)^{\lambda_{1} \lambda_{2}},\left(\mu_{\alpha}^{+}\right)^{\lambda_{1} \lambda_{2}}\right],\right. \\
& {\left.\left[1-\left(1-v_{\alpha}^{-}\right)^{\lambda_{1} \lambda_{2}}, 1-\left(1-v_{\alpha}^{+}\right)^{\lambda_{1} \lambda_{2}}\right]\right) \mid } \\
&\alpha \in \tilde{h}\},\left(\widetilde{h}^{\lambda_{1}}\right)^{\lambda_{2}} \\
&=\left\{\left(\left[\left(\mu_{\alpha}^{-}\right)^{\lambda_{1}},\left(\mu_{\alpha}^{+}\right)^{\lambda_{1}}\right],\right.\right. \\
& {\left.\left[1-\left(1-v_{\alpha}^{-}\right)^{\lambda_{1}}, 1-\left(1-v_{\alpha}^{+}\right)^{\lambda_{1}}\right]\right) \mid } \\
&\alpha \in \tilde{h}\})^{\lambda_{2}}\left\{\left(\left[\left(\mu_{\alpha}^{-}\right)^{\lambda_{1}}\right)^{\lambda_{2}},\left(\left(\mu_{\alpha}^{+}\right)^{\lambda_{1}}\right)^{\lambda_{2}}\right],\right. \\
& {\left[1-\left(1-1+\left(1-v_{\alpha}^{-}\right)^{\lambda_{1}}\right)^{\lambda_{2}},\right.} \\
&\alpha \in \tilde{h}\} . \\
&\left.\left.1-\left(1-1+\left(1-v_{\alpha}^{+}\right)^{\lambda_{1}}\right)^{\lambda_{2}}\right]\right) \mid \\
&\alpha \in \tilde{h}\} \\
&=\left\{\left(\left[\left(\mu_{\alpha}^{-}\right)^{\lambda_{1} \lambda_{2}},\left(\mu_{\alpha}^{+}\right)^{\lambda_{1} \lambda_{2}}\right],\right.\right. \\
& {\left.\left[1-\left(1-v_{\alpha}^{-}\right)^{\lambda_{1} \lambda_{2}}, 1-\left(1-v_{\alpha}^{+}\right)^{\lambda_{1} \lambda_{2}}\right]\right) \mid }
\end{aligned}
$$

Thus, we have that $\widetilde{h}^{\lambda_{1} \lambda_{2}}=\left(\widetilde{h}^{\lambda_{1}}\right)^{\lambda_{2}}$.

To compare the IVIHFEs, we define the following comparison laws.
Definition 10. For an IVIHFE $\widetilde{h}, s(\widetilde{h})=\sum_{\alpha \in \tilde{h}} s(\alpha) / \# \tilde{h}$ is called the score function of $\widetilde{h}$, where $\# \widetilde{h}$ is the number of the elements in $\widetilde{h} . h(\widetilde{h})=\sum_{\alpha \in \widetilde{h}} h(\alpha) / \# \widetilde{h}$ is called the accuracy function of $\widetilde{h}$. For any two IVIHFEs $\widetilde{h}_{1}$ and $\widetilde{h}_{2}$,

(1) if $s\left(\widetilde{h}_{1}\right)>s\left(\widetilde{h}_{2}\right)$, then $\widetilde{h}_{1}>\widetilde{h}_{2}$;

(2) if $s\left(\widetilde{h}_{1}\right)=s\left(\widetilde{h}_{2}\right)$, then the following hold.
(a) If $h\left(\widetilde{h}_{1}\right)>h\left(\widetilde{h}_{2}\right)$, then $\widetilde{h}_{1}>\widetilde{h}_{2}$.
(b) If $h\left(\widetilde{h}_{1}\right)=h\left(\widetilde{h}_{2}\right)$, then $\widetilde{h}_{1}=\widetilde{h}_{2}$.
(c) If $h\left(\widetilde{h}_{2}\right)>h\left(\widetilde{h}_{1}\right)$, then $\widetilde{h}_{2}>\widetilde{h}_{1}$.

\section{Aggregation Operators for Interval-Valued Intuitionistic Hesitant Fuzzy Information}

In the current section, we propose a series of operators for aggregating the interval-valued intuitionistic hesitant fuzzy information and investigate some desired properties of these operators.

\subsection{The IVIHFWA, IVIHFWG, GIVIHFWA, and GIVIHFWG Operators}

Definition 11. Let $\widetilde{h}_{i}(i=1,2, \ldots, n)$ be a collection of IVIHFEs, and let $w=\left(w_{1}, w_{2}, \ldots, w_{n}\right)^{T}$ be the weight vector of $\widetilde{h}_{i}(i=1,2, \ldots, n)$ with $w_{i} \in[0,1]$ and $\sum_{i=1}^{n} w_{i}=1$. An interval-valued intuitionistic hesitant fuzzy weighted averaging (IVIHFWA) operator is a mapping $\widetilde{H}^{n} \rightarrow \widetilde{H}$ such that

$$
\operatorname{IVIHFWA}\left(\widetilde{h}_{1}, \widetilde{h}_{2}, \ldots, \widetilde{h}_{n}\right)=\bigoplus_{i=1}^{n}\left(w_{i} \widetilde{h}_{i}\right) \text {. }
$$

If $w=(1 / n, 1 / n, \ldots, 1 / n)^{T}$ especially, then the IVIHFWA operator reduces to the interval-valued intuitionistic hesitant fuzzy averaging (IVIHFA) operator:

$$
\operatorname{IVIHFA}\left(\widetilde{h}_{1}, \widetilde{h}_{2}, \ldots, \widetilde{h}_{n}\right)=\bigoplus_{i=1}^{n}\left(\frac{1}{n} \widetilde{h}_{i}\right) .
$$

Theorem 12. Let $\widetilde{h}_{i}(i=1,2, \ldots, n)$ be a collection of IVIHFEs. Then, their aggregated value calculated using the IVIHFWA operator is an IVIHFE and

$$
\begin{gathered}
\operatorname{IVIHFWA}\left(\widetilde{h}_{1}, \widetilde{h}_{2}, \ldots, \widetilde{h}_{n}\right) \\
=\left\{\left(\left[1-\prod_{i=1}^{n}\left(1-\mu_{\alpha_{i}}^{-}\right)^{w_{i}}, 1-\prod_{i=1}^{n}\left(1-\mu_{\alpha_{i}}^{+}\right)^{w_{i}}\right],\right.\right. \\
\left.\left[\prod_{i=1}^{n}\left(v_{\alpha_{i}}^{-}\right)^{w_{i}}, \prod_{i=1}^{n}\left(v_{\alpha_{i}}^{+}\right)^{w_{i}}\right]\right) \mid \\
\left.\alpha_{1} \in \widetilde{h}_{1}, \alpha_{2} \in \widetilde{h}_{2}, \ldots, \alpha_{n} \in \widetilde{h}_{n}\right\} .
\end{gathered}
$$


8

Journal of Applied Mathematics

Proof. The first result follows quickly from Definition 11 and Theorem 8. In the following, we prove the second result by using mathematical induction on $n$. First, we show that (16) holds for $n=2$.

Because

$$
\begin{gathered}
w_{1} \widetilde{h}_{1}=\left\{\left(\left[1-\left(1-\mu_{\alpha_{1}}^{-}\right)^{w_{1}}, 1-\left(1-\mu_{\alpha_{1}}^{+}\right)^{w_{1}}\right],\right.\right. \\
\left.\left.\left[\left(v_{\alpha_{1}}^{-}\right)^{w_{1}},\left(\nu_{\alpha_{1}}^{+}\right)^{w_{1}}\right]\right) \mid \alpha_{1} \in \widetilde{h}_{1}\right\}, \\
w_{2} \widetilde{h}_{2}=\left\{\left(\left[1-\left(1-\mu_{\alpha_{2}}^{-}\right)^{w_{2}}, 1-\left(1-\mu_{\alpha_{2}}^{+}\right)^{w_{2}}\right],\right.\right. \\
\left.\left.\left[\left(v_{\alpha_{2}}^{-}\right)^{w_{2}},\left(\nu_{\alpha_{2}}^{+}\right)^{w_{2}}\right]\right) \mid \alpha_{2} \in \widetilde{h}_{2}\right\},
\end{gathered}
$$

we have

$$
\begin{aligned}
& w_{1} \tilde{h}_{1} \oplus w_{2} \tilde{h}_{2} \\
& =\left\{\left(\left[1-\left(1-\mu_{\alpha_{1}}^{-}\right)^{w_{1}}, 1-\left(1-\mu_{\alpha_{1}}^{+}\right)^{w_{1}}\right],\right.\right. \\
& \left.\left.\left[\left(v_{\alpha_{1}}^{-}\right)^{w_{1}},\left(v_{\alpha_{1}}^{+}\right)^{w_{1}}\right]\right) \mid \alpha_{1} \in \widetilde{h}_{1}\right\} \\
& \oplus\left\{\left(\left[1-\left(1-\mu_{\alpha_{2}}^{-}\right)^{w_{2}}, 1-\left(1-\mu_{\alpha_{2}}^{+}\right)^{w_{2}}\right]\right.\right. \text {, } \\
& \left.\left.\left[\left(v_{\alpha_{2}}^{-}\right)^{w_{2}},\left(v_{\alpha_{2}}^{+}\right)^{w_{2}}\right]\right) \mid \alpha_{2} \in \tilde{h}_{2}\right\} \\
& =\left\{\left(\left[1-\left(1-\mu_{\alpha_{1}}^{-}\right)^{w_{1}}+1-\left(1-\mu_{\alpha_{2}}^{-}\right)^{w_{2}}\right.\right.\right. \\
& -\left(1-\left(1-\mu_{\alpha_{1}}^{-}\right)^{w_{1}}\right)\left(1-\left(1-\mu_{\alpha_{2}}^{-}\right)^{w_{2}}\right) \text {, } \\
& 1-\left(1-\mu_{\alpha_{1}}^{+}\right)^{w_{1}}+1-\left(1-\mu_{\alpha_{2}}^{+}\right)^{w_{2}} \\
& \left.-\left(1-\left(1-\mu_{\alpha_{1}}^{+}\right)^{w_{1}}\right)\left(1-\left(1-\mu_{\alpha_{2}}^{+}\right)^{w_{2}}\right)\right] \text {, } \\
& \left.\left[\left(\nu_{\alpha_{1}}^{-}\right)^{w_{1}}\left(\nu_{\alpha_{2}}^{-}\right)^{w_{2}},\left(\nu_{\alpha_{1}}^{+}\right)^{w_{1}}\left(\nu_{\alpha_{2}}^{+}\right)^{w_{2}}\right]\right) \mid \\
& \left.\alpha_{1} \in \tilde{h}_{1}, \alpha_{2} \in \tilde{h}_{2}\right\} \\
& =\left\{\left(\left[1-\left(1-\mu_{\alpha_{1}}^{-}\right)^{w_{1}}\left(1-\mu_{\alpha_{2}}^{-}\right)^{w_{2}}\right.\right.\right. \text {, } \\
& \left.1-\left(1-\mu_{\alpha_{1}}^{+}\right)^{w_{1}}\left(1-\mu_{\alpha_{2}}^{+}\right)^{w_{2}}\right] \text {, } \\
& \left.\left[\left(\nu_{\alpha_{1}}^{-}\right)^{w_{1}}\left(\nu_{\alpha_{2}}^{-}\right)^{w_{2}},\left(\nu_{\alpha_{1}}^{+}\right)^{w_{1}}\left(\nu_{\alpha_{2}}^{+}\right)^{w_{2}}\right]\right) \text { | } \\
& \left.\alpha_{1} \in \widetilde{h}_{1}, \alpha_{2} \in \widetilde{h}_{2}\right\} \text {. }
\end{aligned}
$$

If (16) holds for $n=k$, in other words, $\operatorname{IVIHFWA}\left(\widetilde{h}_{1}, \widetilde{h}_{2}, \ldots, \widetilde{h}_{k}\right)$

$$
\begin{gathered}
=\left\{\left(\left[1-\prod_{i=1}^{k}\left(1-\mu_{\alpha_{i}}^{-}\right)^{w_{i}}, 1-\prod_{i=1}^{k}\left(1-\mu_{\alpha_{i}}^{+}\right)^{w_{i}}\right],\right.\right. \\
\left.\left[\prod_{i=1}^{k}\left(v_{\alpha_{i}}^{-}\right)^{w_{i}}, \prod_{i=1}^{k}\left(v_{\alpha_{i}}^{+}\right)^{w_{i}}\right]\right) \mid \\
\left.\alpha_{1} \in \widetilde{h}_{1}, \alpha_{2} \in \widetilde{h}_{2}, \ldots, \alpha_{k} \in \widetilde{h}_{k}\right\},
\end{gathered}
$$

then, when $n=k+1$, IVIHFE operations yield

$$
\begin{gathered}
\operatorname{IVIHFWA}\left(\widetilde{h}_{1}, \widetilde{h}_{2}, \ldots, \widetilde{h}_{k}, \widetilde{h}_{k+1}\right) \\
=\bigoplus_{i=1}^{k+1}\left(w_{i} \widetilde{h}_{i}\right)=\left(\bigoplus_{i=1}^{k}\left(w_{i} \widetilde{h}_{i}\right)\right) \oplus\left(w_{k+1} \widetilde{h}_{k+1}\right) \\
=\left\{\left(\left[1-\prod_{i=1}^{k}\left(1-\mu_{\alpha_{i}}^{-}\right)^{w_{i}}, 1-\prod_{i=1}^{k}\left(1-\mu_{\alpha_{i}}^{+}\right)^{w_{i}}\right],\right.\right. \\
\left.\left[\prod_{i=1}^{k}\left(v_{\alpha_{i}}^{-}\right)^{w_{i}}, \prod_{i=1}^{k}\left(v_{\alpha_{i}}^{+}\right)^{w_{i}}\right]\right) \mid
\end{gathered}
$$$$
\left.\alpha_{1} \in \widetilde{h}_{1}, \alpha_{2} \in \widetilde{h}_{2}, \ldots, \alpha_{k} \in \widetilde{h}_{k}\right\}
$$

$\oplus\left\{\left(\left[1-\left(1-\mu_{\alpha_{k+1}}^{-}\right)^{w_{k+1}}, 1-\left(1-\mu_{\alpha_{k+1}}^{+}\right)^{w_{k+1}}\right]\right.\right.$,

$$
\left.\left.\left[\left(\nu_{\alpha_{k+1}}^{-}\right)^{w_{k+1}},\left(\nu_{\alpha_{k+1}}^{+}\right)^{w_{k+1}}\right]\right) \mid \alpha_{k+1} \in \widetilde{h}_{k+1}\right\}
$$$$
=\left\{\left(\left[1-\prod_{i=1}^{k}\left(1-\mu_{\alpha_{i}}^{-}\right)^{w_{i}}+1-\left(1-\mu_{\alpha_{k+1}}^{-}\right)^{w_{k+1}}\right.\right.\right.
$$$$
-\left(1-\prod_{i=1}^{k}\left(1-\mu_{\alpha_{i}}^{-}\right)^{w_{i}}\right)\left(1-\left(1-\mu_{\alpha_{k+1}}^{-}\right)^{w_{k+1}}\right),
$$$$
1-\prod_{i=1}^{k}\left(1-\mu_{\alpha_{i}}^{+}\right)^{w_{i}}+1-\left(1-\mu_{\alpha_{k+1}}^{+}\right)^{w_{k+1}}
$$$$
\left.-\left(1-\prod_{i=1}^{k}\left(1-\mu_{\alpha_{i}}^{+}\right)^{w_{i}}\right)\left(1-\left(1-\mu_{\alpha_{k+1}}^{+}\right)^{w_{k+1}}\right)\right],
$$

$\left[\left(\prod_{i=1}^{k}\left(v_{\alpha_{i}}^{-}\right)^{w_{i}}\right)\left(\left(\nu_{\alpha_{k+1}}^{-}\right)^{w_{k+1}}\right)\right.$,

$$
\left.\left.\left(\prod_{i=1}^{k}\left(v_{\alpha_{i}}^{+}\right)^{w_{i}}\right)\left(\left(v_{\alpha_{k+1}}^{+}\right)^{w_{k+1}}\right)\right]\right) \mid
$$$$
\left.\alpha_{1} \in \widetilde{h}_{1}, \alpha_{2} \in \widetilde{h}_{2}, \ldots, \alpha_{k} \in \tilde{h}_{k}, \alpha_{k+1} \in \widetilde{h}_{k+1}\right\}
$$

$$
\begin{gathered}
=\left\{\left(\left[1-\prod_{i=1}^{k+1}\left(1-\mu_{\alpha_{i}}^{-}\right)^{w_{i}}, 1-\prod_{i=1}^{k+1}\left(1-\mu_{\alpha_{i}}^{+}\right)^{w_{i}}\right],\right.\right. \\
\left.\left[\prod_{i=1}^{k+1}\left(v_{\alpha_{i}}^{-}\right)^{w_{i}}, \prod_{i=1}^{k+1}\left(v_{\alpha_{i}}^{+}\right)^{w_{i}}\right]\right) \mid \\
\left.\alpha_{1} \in \widetilde{h}_{1}, \alpha_{2} \in \widetilde{h}_{2}, \ldots, \alpha_{k} \in \widetilde{h}_{k}, \alpha_{k+1} \in \widetilde{h}_{k+1}\right\} .
\end{gathered}
$$

In other words, (16) holds for $n=k+1$. Equation (16) therefore holds for all $n$.

This completes the proof of Theorem 12 . 
Definition 13. Let $\widetilde{h}_{i}(i=1,2, \ldots, n)$ be a collection of IVIHFEs, and let $w=\left(w_{1}, w_{2}, \ldots, w_{n}\right)^{T}$ be the weight vector of $\widetilde{h}_{i}(i=1,2, \ldots, n)$ with $w_{i} \in[0,1]$ and $\sum_{i=1}^{n} w_{i}=$ 1. An interval-valued intuitionistic hesitant fuzzy weighted geometric (IVIHFWG) operator is a mapping $\widetilde{H}^{n} \rightarrow \widetilde{H}$ such that

$$
\operatorname{IVIHFWG}\left(\widetilde{h}_{1}, \widetilde{h}_{2}, \ldots, \widetilde{h}_{n}\right)=\bigotimes_{i=1}^{n}\left(\widetilde{h}_{i}^{w_{i}}\right) .
$$

Especially, if $w=(1 / n, 1 / n, \ldots, 1 / n)^{T}$, then the IVIHFWG operator reduces to the interval-valued intuitionistic hesitant fuzzy averaging (IVIHFA) operator:

$$
\operatorname{IVIHFG}\left(\widetilde{h}_{1}, \widetilde{h}_{2}, \ldots, \widetilde{h}_{n}\right)=\bigotimes_{i=1}^{n}\left(\widetilde{h}_{i}^{1 / n}\right) .
$$

Theorem 14. Let $\widetilde{h}_{i}(i=1,2, \ldots, n)$ be a collection of IVIHFEs. Then, their aggregated value calculated using the IVIHFWG operator is an IVIHFE, and

$$
\begin{aligned}
& \text { IVIHFWG }\left(\widetilde{h}_{1}, \widetilde{h}_{2}, \ldots, \widetilde{h}_{n}\right) \\
& =\left\{\left(\left[\prod_{i=1}^{n}\left(\mu_{\alpha_{i}}^{-}\right)^{w_{i}}, \prod_{i=1}^{n}\left(\mu_{\alpha_{i}}^{+}\right)^{w_{i}}\right],\right.\right. \\
& \left.\quad\left[1-\prod_{i=1}^{n}\left(1-v_{\alpha_{i}}^{-}\right)^{w_{i}}, 1-\prod_{i=1}^{n}\left(1-v_{\alpha_{i}}^{+}\right)^{w_{i}}\right]\right) \mid \\
& \left.\alpha_{1} \in \widetilde{h}_{1}, \alpha_{2} \in \widetilde{h}_{2}, \ldots, \alpha_{n} \in \widetilde{h}_{n}\right\} .
\end{aligned}
$$

In the following, by combining the IVIHFWA and IVIHFWG operators with the generalized mean [12], we develop the generalized interval-valued intuitionistic hesitant fuzzy weighted averaging (GIVIHFWA) operator and the generalized interval-valued intuitionistic hesitant fuzzy weighted geometric (GIVIHFWG) operator, respectively. The main characteristic of the GIVIHFWA and GIVIHFWG operators is that they have an additional parameter $\lambda$ controlling the power to which the argument values are raised. Different from the IVIHFWA and IVIHFWG operators, the GIVIHFWA and GIVIHFWG operators extend them with addition of a parameter controlling the power to which the argument values are raised. When we use different choices of the parameters $\lambda$, we will get some special cases.

Definition 15. Let $\widetilde{h}_{i}(i=1,2, \ldots, n)$ be a collection of IVIHFEs, and let $w=\left(w_{1}, w_{2}, \ldots, w_{n}\right)^{T}$ be the weight vector of $\widetilde{h}_{i}(i=1,2, \ldots, n)$ with $w_{i} \in[0,1]$ and $\sum_{i=1}^{n} w_{i}=1$.

(1) A generalized interval-valued intuitionistic hesitant fuzzy weighted averaging (GIVIHFWA) operator is a mapping $\widetilde{H}^{n} \rightarrow \widetilde{H}$, where

$$
\operatorname{GIVIHFWA}_{\lambda}\left(\widetilde{h}_{1}, \tilde{h}_{2}, \ldots, \tilde{h}_{n}\right)=\left(\bigoplus_{i=1}^{n}\left(w_{i} \widetilde{h}_{i}^{\lambda}\right)\right)^{1 / \lambda}
$$

with $\lambda>0$.
(2) A generalized interval-valued intuitionistic hesitant fuzzy weighted geometric (GIVIHFWG) operator is a mapping $\widetilde{H}^{n} \rightarrow \widetilde{H}$, where

$$
\operatorname{GIVIHFWG}_{\lambda}\left(\widetilde{h}_{1}, \widetilde{h}_{2}, \ldots, \widetilde{h}_{n}\right)=\frac{1}{\lambda}\left(\bigotimes_{i=1}^{n}\left(\lambda \widetilde{h}_{i}\right)^{w_{i}}\right)
$$

with $\lambda>0$.

If $\lambda=1$, then the GIVIHFWA operator reduces to the IVIHFWA operator and the GIVIHFWG operator reduces to the IVIHFWG operator.

Using IVIHFE operations and mathematical induction on $n,(24)$ and (25) can be transformed into the following forms:

$$
\begin{aligned}
& \operatorname{GIVIHFWA}_{\lambda}\left(\widetilde{h}_{1}, \tilde{h}_{2}, \ldots, \widetilde{h}_{n}\right) \\
& =\left\{\left(\left[\left(1-\prod_{i=1}^{n}\left(1-\left(\mu_{\alpha_{i}}^{-}\right)^{\lambda}\right)^{w_{i}}\right)^{1 / \lambda},\right.\right.\right. \\
& \left.\left(1-\prod_{i=1}^{n}\left(1-\left(\mu_{\alpha_{i}}^{+}\right)^{\lambda}\right)^{w_{i}}\right)^{1 / \lambda}\right] \\
& {\left[1-\left(1-\prod_{i=1}^{n}\left(1-\left(1-\nu_{\alpha_{i}}^{-}\right)^{\lambda}\right)^{w_{i}}\right)^{1 / \lambda},\right.} \\
& \left.\left.1-\left(1-\prod_{i=1}^{n}\left(1-\left(1-v_{\alpha_{i}}^{+}\right)^{\lambda}\right)^{w_{i}}\right)^{1 / \lambda}\right]\right) \mid \\
& \left.\alpha_{1} \in \tilde{h}_{1}, \alpha_{2} \in \tilde{h}_{2}, \ldots, \alpha_{n} \in \tilde{h}_{n}\right\} \text {, } \\
& \operatorname{GIVIHFWG}_{\lambda}\left(\widetilde{h}_{1}, \widetilde{h}_{2}, \ldots, \widetilde{h}_{n}\right) \\
& =\left\{\left(\left[1-\left(1-\prod_{i=1}^{n}\left(1-\left(1-\mu_{\alpha_{i}}^{-}\right)^{\lambda}\right)^{w_{i}}\right)^{1 / \lambda},\right.\right.\right. \\
& \left.1-\left(1-\prod_{i=1}^{n}\left(1-\left(1-\mu_{\alpha_{i}}^{+}\right)^{\lambda}\right)^{w_{i}}\right)^{1 / \lambda}\right] \\
& {\left[\left(1-\prod_{i=1}^{n}\left(1-\left(\nu_{\alpha_{i}}^{-}\right)^{\lambda}\right)^{w_{i}}\right)^{1 / \lambda},\right.} \\
& \left.\left.\left(1-\prod_{i=1}^{n}\left(1-\left(v_{\alpha_{i}}^{+}\right)^{\lambda}\right)^{w_{i}}\right)^{1 / \lambda}\right]\right) \mid \\
& \left.\alpha_{1} \in \widetilde{h}_{1}, \alpha_{2} \in \widetilde{h}_{2}, \ldots, \alpha_{n} \in \widetilde{h}_{n}\right\} \text {. }
\end{aligned}
$$

Example 16. Suppose that $\widetilde{h}_{1}=\{([0.2,0.3],[0.5,0.6])$, $([0.5,08],[0.1,0.2]),([0.1,0.3],[0.4,0.5])\}, \widetilde{h}_{2}=\{([0.4,0.6]$, $[0.3,0.4]),([0.3,0.5],[0.1,0.2])\}$, and $\widetilde{h}_{3}=\{([0.5,0.5]$, $[0.2,0.3]),([0.2,0.4],[0.3,0.6]),([0.8,0.9],[0.1,0.1])\}$ are three IVIHFEs, and $w=(0.6,0.3,0.1)^{T}$ is their weight vector. Then, by Definition 15, we can obtain 


$$
\begin{aligned}
& \operatorname{GIVIHFWA}_{1}\left(\widetilde{h}_{1}, \widetilde{h}_{2}, \widetilde{h}_{3}\right) \\
& =\operatorname{IVIHFWA}\left(\widetilde{h}_{1}, \widetilde{h}_{2}, \widetilde{h}_{3}\right) \\
& =\left\{\begin{array}{l}
([0.2998,0.4278],[0.3914,0.4957]),([0.2661,0.4172],[0.4076,0.5313]),([0.3611,0.5128],[0.3652,0.4441]), \\
([0.2667,0.3881],[0.2815,0.4026]),([0.2314,0.3769],[0.2932,0.4315]),([0.3309,0.4791],[0.2627,0.3607]), \\
([0.4719,0.7301],[0.1490,0.2564]),([0.4465,0.7252],[0.1552,0.2748]),([0.5181,0.7703],[0.1390,0.2297]), \\
([0.4469,0.7115],[0.1072,0.2083]),([0.4203,0.7062],[0.1116,0.2232]),([0.4953,0.7544],[0.1000,0.1866]), \\
([0.2486,0.4278],[0.3424,0.4443]),([0.2124,0.4172],[0.3565,0.4762]),([0.3144,0.5128],[0.3194,0.3981]), \\
([0.2130,0.3881],[0.2462,0.3609]),([0.1751,0.3769],[0.2564,0.3868]),([0.2819,0.4791],[0.2297,0.3234])
\end{array}\right\}, \\
& \operatorname{GIVIHFWA}_{5}\left(\widetilde{h}_{1}, \widetilde{h}_{2}, \widetilde{h}_{3}\right) \\
& \left.\int([0.3645,0.4909],[0.3596,0.4559]),([0.3191,0.4835],[0.3843,0.5008]),([0.5306,0.6415],[0.3150,0.3536]),\right) \\
& ([0.3329,0.4262],[0.2255,0.3312]),([0.2488,0.4125],[0.2344,0.3471]),([0.5248,0.6250],[0.2070,0.2803]), \\
& =\left\{\begin{array}{l}
([0.4782,0.7475],[0.1410,0.2453]),([0.4658,0.7464],[0.1452,0.2536]),([0.5696,0.7842],[0.1317,0.2158]), \\
([0.4690,0.7400],[0.1064,0.2072]),([0.4555,0.7389],[0.1094,0.2134]),([0.5653,0.7786],[0.1000,0.1847]),
\end{array}\right\} \\
& ([0.3624,0.4909],[0.3314,0.4306]),([0.3154,0.4835],[0.3512,0.4661]),([0.5302,0.6415],[0.2939,0.3411]), \\
& ([0.3298,0.4262],[0.2142,0.3204]),([0.2382,0.4125],[0.2223,0.3351]),([0.5243,0.6250],[0.1972,0.2728])] \\
& \operatorname{GIVIHFWG}_{1}\left(\widetilde{h}_{1}, \widetilde{h}_{2}, \widetilde{h}_{3}\right) \\
& =\operatorname{IVIHFWG}\left(\widetilde{h}_{1}, \widetilde{h}_{2}, \widetilde{h}_{3}\right) \\
& =\left\{\begin{array}{l}
([0.2699,0.3887],[0.4203,0.5223]),([0.2462,0.3801],[0.4280,0.5483]),([0.2828,0.4122],[0.4134,0.5101]), \\
([0.2475,0.3680],[0.3749,0.4792]),([0.2259,0.3599],[0.3832,0.5075]),([0.2595,0.3903],[0.3675,0.4659]), \\
([0.4676,0.7002],[0.1751,0.2759]),([0.4267,0.6847],[0.1861,0.3153]),([0.4901,0.7425],[0.1654,0.2575]), \\
([0.4290,0.6629],[0.1105,0.2106]),([0.3914,0.6483],[0.1223,0.2536]),([0.4496,0.7030],[0.1000,0.1905]), \\
([0.1780,0.3887],[0.3533,0.4538]),([0.1625,0.3801],[0.3618,0.4835]),([0.1866,0.4122],[0.3456,0.4399]), \\
([0.1633,0.3680],[0.3026,0.4046]),([0.1490,0.3599],[0.3119,0.4370]),([0.1712,0.3903],[0.2944,0.3894])
\end{array}\right\}, \\
& \text { GIVIHFWG }_{5}\left(\widetilde{h}_{1}, \widetilde{h}_{2}, \widetilde{h}_{3}\right) \\
& =\left\{\begin{array}{l}
([0.2525,0.3568],[0.4555,0.5505]),([0.2357,0.3517],[0.4565,0.5658]),([0.2541,0.3601],[0.4554,0.5500]), \\
([0.2382,0.3502],[0.4522,0.5442]),([0.2229,0.3454],[0.4532,0.5602]),([0.2395,0.3534],[0.4520,0.5437]), \\
([0.4611,0.6355],[0.2382,0.3230]),([0.3971,0.5920],[0.2501,0.4080]),([0.4684,0.6815],[0.2363,0.3185]), \\
([0.4051,0.5817],[0.1326,0.2213]),([0.3596,0.5540],[0.1907,0.3840]),([0.4098,0.6046],[0.1000,0.1960]), \\
([0.1551,0.3568],[0.3698,0.4667]),([0.1472,0.3517],[0.3720,0.4952]),([0.1558,0.3601],[0.3694,0.4657]), \\
([0.1484,0.3502],[0.3617,0.4536]),([0.1410,0.3454],[0.3641,0.4851]),([0.1491,0.3534],[0.3613,0.4525])
\end{array}\right\} .
\end{aligned}
$$

Theorem 17. Let $\widetilde{h}_{i}(i=1,2, \ldots, n)$ be a collection of IVIHFEs, $\lambda>0$, and $w=\left(w_{1}, w_{2}, \ldots, w_{n}\right)^{T}$ is the weight vector of $\widetilde{h}_{i}(i=1,2, \ldots, n)$ with $w_{i} \in[0,1]$ and $\sum_{i=1}^{n} w_{i}=1$. Then, the GIVIHFWA operator GIVIHFWA $A_{\lambda}\left(\widetilde{h}_{1}, \widetilde{h}_{2}, \ldots, \widetilde{h}_{n}\right)$ is monotonically increasing with respect to the parameter $\lambda$.
Proof. According to the proof of Theorem 3.8 in [13], we can obtain that $\left(1-\prod_{i=1}^{n}\left(1-\left(\mu_{\alpha_{i}}^{-}\right)^{\lambda}\right)^{w_{i}}\right)^{1 / \lambda}$ and $\left(1-\prod_{i=1}^{n}\left(1-\left(\mu_{\alpha_{i}}^{+}\right)^{\lambda}\right)^{w_{i}}\right)^{1 / \lambda}$ are monotonically increasing with respect to the parameter $\lambda$. Furthermore, $1-(1-$ $\left.\prod_{i=1}^{n}\left(1-\left(1-v_{\alpha_{i}}^{-}\right)^{\lambda}\right)^{w_{i}}\right)^{1 / \lambda}$ and $1-\left(1-\prod_{i=1}^{n}\left(1-\left(1-v_{\alpha_{i}}^{+}\right)^{\lambda}\right)^{w_{i}}\right)^{1 / \lambda}$ are monotonically decreasing with respect to the parameter $\lambda$. Therefore, by Definition 10,

$$
\begin{aligned}
& s\left(\operatorname{GIVIHFWA}_{\lambda}\left(\tilde{h}_{1}, \tilde{h}_{2}, \ldots, \tilde{h}_{n}\right)\right) \\
&=\left(\frac { 1 } { 2 } \sum _ { \alpha _ { 1 } \in \tilde { h } _ { 1 } , \alpha _ { 2 } \in \tilde { h } _ { 2 } , \ldots , \alpha _ { n } \in \tilde { h } _ { n } } \left(\left(1-\prod_{i=1}^{n}\left(1-\left(\mu_{\alpha_{i}}^{-}\right)^{\lambda}\right)^{w_{i}}\right)^{1 / \lambda}-1+\left(1-\prod_{i=1}^{n}\left(1-\left(1-v_{\alpha_{i}}^{-}\right)^{\lambda}\right)^{w_{i}}\right)^{1 / \lambda}\right.\right. \\
&\left.\left.+\left(1-\prod_{i=1}^{n}\left(1-\left(\mu_{\alpha_{i}}^{+}\right)^{\lambda}\right)^{w_{i}}\right)^{1 / \lambda}-1+\left(1-\prod_{i=1}^{n}\left(1-\left(1-v_{\alpha_{i}}^{+}\right)^{\lambda}\right)^{w_{i}}\right)^{1 / \lambda}\right)\right) \times\left(\prod_{i=1}^{n} \# \widetilde{h}_{i}\right)^{-1}
\end{aligned}
$$


is monotonically increasing with respect to the parameter $\lambda$, which implies that GIVIHFWA ${ }_{\lambda}\left(\widetilde{h}_{1}, \widetilde{h}_{2}, \ldots, \widetilde{h}_{n}\right)$ is monotonically increasing with respect to the parameter $\lambda$.

Theorem 18. Let $\widetilde{h}_{i}(i=1,2, \ldots, n)$ be a collection of IVIHFEs, $\lambda>0$, and $w=\left(w_{1}, w_{2}, \ldots, w_{n}\right)^{T}$ is the weight vector of $\widetilde{h}_{i}(i=1,2, \ldots, n)$ with $w_{i} \in[0,1]$ and $\sum_{i=1}^{n} w_{i}=1$. Then, the GIVIHFWG operator GIVIHFWG $G_{\lambda}\left(\widetilde{h}_{1}, \widetilde{h}_{2}, \ldots, \widetilde{h}_{n}\right)$ is monotonically decreasing with respect to the parameter $\lambda$.
Proof. According to the proof of Theorem 17, we can obtain that $1-\left(1-\prod_{i=1}^{n}\left(1-\left(1-\mu_{\alpha_{i}}^{-}\right)^{\lambda}\right)^{w_{i}}\right)^{1 / \lambda}$ and $1-\left(1-\prod_{i=1}^{n}(1-\right.$ $\left.\left.\left(1-\mu_{\alpha_{i}}^{+}\right)^{\lambda}\right)^{w_{i}}\right)^{1 / \lambda}$ are monotonically decreasing with respect to the parameter $\lambda$. Furthermore, $\left(1-\prod_{i=1}^{n}\left(1-\left(\nu_{\alpha_{i}}^{-}\right)^{\lambda}\right)^{w_{i}}\right)^{1 / \lambda}$ and $\left(1-\prod_{i=1}^{n}\left(1-\left(\nu_{\alpha_{i}}^{+}\right)^{\lambda}\right)^{w_{i}}\right)^{1 / \lambda}$ are monotonically increasing with respect to the parameter $\lambda$. Therefore, by Definition 10,

$$
\begin{aligned}
& s\left(\operatorname{GIVIHFWG}_{\lambda}\left(\widetilde{h}_{1}, \widetilde{h}_{2}, \ldots, \tilde{h}_{n}\right)\right) \\
& =\left(\frac { 1 } { 2 } \sum _ { \alpha _ { 1 } \in \tilde { h } _ { 1 } , \alpha _ { 2 } \in \tilde { h } _ { 2 } , \ldots , \alpha _ { n } \in \tilde { h } _ { n } } \left(1-\left(1-\prod_{i=1}^{n}\left(1-\left(1-\mu_{\alpha_{i}}^{-}\right)^{\lambda}\right)^{w_{i}}\right)^{1 / \lambda}-\left(1-\prod_{i=1}^{n}\left(1-\left(v_{\alpha_{i}}^{-}\right)^{\lambda}\right)^{w_{i}}\right)^{1 / \lambda}\right.\right. \\
& \left.\left.+1-\left(1-\prod_{i=1}^{n}\left(1-\left(1-\mu_{\alpha_{i}}^{+}\right)^{\lambda}\right)^{w_{i}}\right)^{1 / \lambda}-\left(1-\prod_{i=1}^{n}\left(1-\left(v_{\alpha_{i}}^{+}\right)^{\lambda}\right)^{w_{i}}\right)^{1 / \lambda}\right)\right) \times\left(\prod_{i=1}^{n} \# \widetilde{h}_{i}\right)^{-1}
\end{aligned}
$$

is monotonically decreasing with respect to the parameter $\lambda$, which implies that GIVIHFWG ${ }_{\lambda}\left(\widetilde{h}_{1}, \widetilde{h}_{2}, \ldots, \widetilde{h}_{n}\right)$ is monotonically decreasing with respect to the parameter $\lambda$.

Lemma 19 (see $[14,15])$. Let $x_{i}>0, \lambda_{i}>0, i=1,2, \ldots, n$, and $\sum_{i=1}^{n} \lambda_{i}=1$, then

$$
\prod_{i=1}^{n}\left(x_{i}\right)^{\lambda_{i}} \leq \sum_{i=1}^{n} \lambda_{i} x_{i}
$$

with equality if and only if $x_{1}=x_{2}=\cdots=x_{n}$.

Theorem 20. Let $\widetilde{h}_{i}(i=1,2, \ldots, n)$ be a collection of IVIHFEs having the weight vector $w=\left(w_{1}, w_{2}, \ldots, w_{n}\right)^{T}$ such that $w_{i} \in$ $[0,1]$ and $\sum_{i=1}^{n} w_{i}=1, \lambda>0$; then

$$
\operatorname{IVIHFWG}\left(\widetilde{h}_{1}, \widetilde{h}_{2}, \ldots, \widetilde{h}_{n}\right) \leq \operatorname{GIVIHFWA} A_{\lambda}\left(\widetilde{h}_{1}, \widetilde{h}_{2}, \ldots, \widetilde{h}_{n}\right) .
$$

Proof. For any $\alpha_{1} \in \widetilde{h}_{1}, \alpha_{2} \in \widetilde{h}_{2}, \ldots, \alpha_{n} \in \widetilde{h}_{n}$, from Lemma 19, we have

$$
\begin{aligned}
\prod_{i=1}^{n}\left(\mu_{\alpha_{i}}^{-}\right)^{w_{i}} & =\left(\prod_{i=1}^{n}\left(\left(\mu_{\alpha_{i}}^{-}\right)^{\lambda}\right)^{w_{i}}\right)^{1 / \lambda} \\
& \leq\left(\sum_{i=1}^{n} w_{i}\left(\mu_{\alpha_{i}}^{-}\right)^{\lambda}\right)^{1 / \lambda} \\
& =\left(1-\sum_{i=1}^{n} w_{i}\left(1-\left(\mu_{\alpha_{i}}^{-}\right)^{\lambda}\right)\right)^{1 / \lambda} \\
& \leq\left(1-\prod_{i=1}^{n}\left(1-\left(\mu_{\alpha_{i}}^{-}\right)^{\lambda}\right)^{w_{i}}\right)^{1 / \lambda},
\end{aligned}
$$

$$
\begin{aligned}
\prod_{i=1}^{n}\left(\mu_{\alpha_{i}}^{+}\right)^{w_{i}} & =\left(\prod_{i=1}^{n}\left(\left(\mu_{\alpha_{i}}^{+}\right)^{\lambda}\right)^{w_{i}}\right)^{1 / \lambda} \\
& \leq\left(\sum_{i=1}^{n} w_{i}\left(\mu_{\alpha_{i}}^{+}\right)^{\lambda}\right)^{1 / \lambda} \\
& =\left(1-\sum_{i=1}^{n} w_{i}\left(1-\left(\mu_{\alpha_{i}}^{+}\right)^{\lambda}\right)\right)^{1 / \lambda} \\
& \leq\left(1-\prod_{i=1}^{n}\left(1-\left(\mu_{\alpha_{i}}^{+}\right)^{\lambda}\right)^{w_{i}}\right)^{1 / \lambda}, \\
& 1-\prod_{i=1}^{n}\left(1-v_{\alpha_{i}}^{-}\right)^{w_{i}} \\
& \geq 1-\left(\prod_{i=1}^{n}\left(\left(1-v_{\alpha_{i}}^{-}\right)^{\lambda}\right)^{w_{i}}\right)^{1 / \lambda} \\
& \geq 1-\left(\sum_{i=1}^{n} w_{i}\left(1-v_{\alpha_{i}}^{-}\right)^{\lambda}\right)^{1 / \lambda} \\
& \geq 1-\left(1-\left(\sum _ { i = 1 } ^ { n } \left(1-\left(\sum_{i=1}^{n} w_{i}\left(1-\left(1-w_{i}\left(1-\left(1-v_{\alpha_{i}}^{-}\right)^{\lambda}\right)\right)\right)^{1 / \lambda}\right.\right.\right.\right. \\
& \left.\left.\left.\geq v_{\alpha_{i}}^{-}\right)^{\lambda}\right)^{w_{i}}\right)^{1 / \lambda} \\
& \\
& 1-\lambda
\end{aligned}
$$


12

Journal of Applied Mathematics

$$
\begin{aligned}
1 & -\prod_{i=1}^{n}\left(1-v_{\alpha_{i}}^{+}\right)^{w_{i}} \\
& \geq 1-\left(\prod_{i=1}^{n}\left(\left(1-v_{\alpha_{i}}^{+}\right)^{\lambda}\right)^{w_{i}}\right)^{1 / \lambda} \\
& \geq 1-\left(\sum_{i=1}^{n} w_{i}\left(1-v_{\alpha_{i}}^{+}\right)^{\lambda}\right)^{1 / \lambda} \\
& \geq 1-\left(1-\left(\sum_{i=1}^{n} w_{i}\left(1-\left(1-v_{\alpha_{i}}^{+}\right)^{\lambda}\right)\right)\right)^{1 / \lambda}
\end{aligned}
$$

$$
\begin{aligned}
& \geq 1-\left(1-\left(\sum_{i=1}^{n} w_{i}\left(1-\left(1-v_{\alpha_{i}}^{+}\right)^{\lambda}\right)\right)\right)^{1 / \lambda} \\
& \geq 1-\left(1-\prod_{i=1}^{n}\left(1-\left(1-v_{\alpha_{i}}^{+}\right)^{\lambda}\right)^{w_{i}}\right)^{1 / \lambda} .
\end{aligned}
$$

By Definition 10, we have

$$
\begin{aligned}
& \left.s \text { IVIHFWG }\left(\widetilde{h}_{1}, \widetilde{h}_{2}, \ldots, \widetilde{h}_{n}\right)\right) \\
& =\left(\frac{1}{2} \sum_{\alpha_{1} \in \tilde{h}_{1}, \alpha_{2} \in \tilde{h}_{2}, \ldots, \alpha_{n} \in \widetilde{h}_{n}}\left[\prod_{i=1}^{n}\left(\mu_{\alpha_{i}}^{-}\right)^{w_{i}}-\left(1-\prod_{i=1}^{n}\left(1-v_{\alpha_{i}}^{-}\right)^{w_{i}}\right)+\prod_{i=1}^{n}\left(\mu_{\alpha_{i}}^{+}\right)^{w_{i}}-\left(1-\prod_{i=1}^{n}\left(1-v_{\alpha_{i}}^{+}\right)^{w_{i}}\right)\right]\right) \\
& \quad \times\left(\#\left(\operatorname{IVIHFWG}\left(\widetilde{h}_{1}, \widetilde{h}_{2}, \ldots, \widetilde{h}_{n}\right)\right)\right)^{-1} \\
& \leq\left(\frac { 1 } { 2 } \sum _ { \alpha _ { 1 } \in \tilde { h } _ { 1 } , \alpha _ { 2 } \in \tilde { h } _ { 2 } , \ldots , \alpha _ { n } \in \tilde { h } _ { n } } \left[\left(1-\prod_{i=1}^{n}\left(1-\left(\mu_{\alpha_{i}}^{-}\right)^{\lambda}\right)^{w_{i}}\right)^{1 / \lambda}-\left(1-\left(1-\prod_{i=1}^{n}\left(1-\left(1-v_{\alpha_{i}}^{-}\right)^{\lambda}\right)^{w_{i}}\right)^{1 / \lambda}\right)\right.\right. \\
& \left.\left.\quad+\left(1-\prod_{i=1}^{n}\left(1-\left(\mu_{\alpha_{i}}^{+}\right)^{\lambda}\right)^{w_{i}}\right)^{1 / \lambda}-\left(1-\left(1-\prod_{i=1}^{n}\left(1-\left(1-v_{\alpha_{i}}^{+}\right)^{\lambda}\right)^{w_{i}}\right)^{1 / \lambda}\right)\right]\right) \\
& \quad \times\left(\#\left(\operatorname{GIVIHFWA}{ }_{\lambda}\left(\widetilde{h}_{1}, \widetilde{h}_{2}, \ldots, \widetilde{h}_{n}\right)\right)\right)^{-1}=s\left(\operatorname{GIVIHFWA}\left(\widetilde{h}_{1}, \widetilde{h}_{2}, \ldots, \widetilde{h}_{n}\right)\right) .
\end{aligned}
$$

If $s\left(\operatorname{IVIHFWG}\left(\widetilde{h}_{1}, \widetilde{h}_{2}, \ldots, \widetilde{h}_{n}\right)\right)<s\left(\operatorname{GIVIHFWA}_{\lambda}\left(\widetilde{h}_{1}, \widetilde{h}_{2}\right.\right.$, $\left.\ldots, \tilde{h}_{n}\right)$ ), then by Definition 10 , we have

$$
<s\left(\operatorname{GIVIHFWA}_{\lambda}\left(\widetilde{h}_{1}, \widetilde{h}_{2}, \ldots, \widetilde{h}_{n}\right)\right) .
$$

If $s\left(\operatorname{IVIHFWG}\left(\widetilde{h}_{1}, \widetilde{h}_{2}, \ldots, \widetilde{h}_{n}\right)\right)=s\left(\operatorname{GIVIHFWA}_{\lambda}\left(\widetilde{h}_{1}, \widetilde{h}_{2}\right.\right.$, $\left.\left.\ldots, \widetilde{h}_{n}\right)\right)$, that is,

$$
\begin{aligned}
& \left(\frac{1}{2} \sum_{\alpha_{1} \in \tilde{h}_{1}, \alpha_{2} \in \widetilde{h}_{2}, \ldots, \alpha_{n} \in \widetilde{h}_{n}}\left[\prod_{i=1}^{n}\left(\mu_{\alpha_{i}}^{-}\right)^{w_{i}}-\left(1-\prod_{i=1}^{n}\left(1-v_{\alpha_{i}}^{-}\right)^{w_{i}}\right)+\prod_{i=1}^{n}\left(\mu_{\alpha_{i}}^{+}\right)^{w_{i}}-\left(1-\prod_{i=1}^{n}\left(1-v_{\alpha_{i}}^{+}\right)^{w_{i}}\right)\right]\right) \\
& \times\left(\#\left(\operatorname{IVIHFWG}\left(\widetilde{h}_{1}, \widetilde{h}_{2}, \ldots, \widetilde{h}_{n}\right)\right)\right)^{-1} \\
& =\left(\frac { 1 } { 2 } \sum _ { \alpha _ { 1 } \in \widetilde { h } _ { 1 } , \alpha _ { 2 } \in \widetilde { h } _ { 2 } , \ldots , \alpha _ { n } \in \tilde { h } _ { n } } \left[\left(1-\prod_{i=1}^{n}\left(1-\left(\mu_{\alpha_{i}}^{-}\right)^{\lambda}\right)^{w_{i}}\right)^{1 / \lambda}-\left(1-\left(1-\prod_{i=1}^{n}\left(1-\left(1-v_{\alpha_{i}}^{-}\right)^{\lambda}\right)^{w_{i}}\right)^{1 / \lambda}\right)\right.\right. \\
& \left.\left.\quad+\left(1-\prod_{i=1}^{n}\left(1-\left(\mu_{\alpha_{i}}^{+}\right)^{\lambda}\right)^{w_{i}}\right)^{1 / \lambda}-\left(1-\left(1-\prod_{i=1}^{n}\left(1-\left(1-v_{\alpha_{i}}^{+}\right)^{\lambda}\right)^{w_{i}}\right)^{1 / \lambda}\right)\right]\right) \\
& \times\left(\#\left(\operatorname{GIVIHFWA}_{\lambda}\left(\widetilde{h}_{1}, \widetilde{h}_{2}, \ldots, \widetilde{h}_{n}\right)\right)\right)^{-1},
\end{aligned}
$$


Journal of Applied Mathematics

13

then, by the conditions that

$$
\begin{aligned}
& \prod_{i=1}^{n}\left(\mu_{\alpha_{i}}^{-}\right)^{w_{i}}-\left(1-\prod_{i=1}^{n}\left(1-v_{\alpha_{i}}^{-}\right)^{w_{i}}\right) \\
& \leq\left(1-\prod_{i=1}^{n}\left(1-\left(\mu_{\alpha_{i}}^{-}\right)^{\lambda}\right)^{w_{i}}\right)^{1 / \lambda} \\
& \quad-\left(1-\left(1-\prod_{i=1}^{n}\left(1-\left(1-v_{\alpha_{i}}^{-}\right)^{\lambda}\right)^{w_{i}}\right)^{1 / \lambda}\right), \\
& \prod_{i=1}^{n}\left(\mu_{\alpha_{i}}^{+}\right)^{w_{i}}-\left(1-\prod_{i=1}^{n}\left(1-v_{\alpha_{i}}^{+}\right)^{w_{i}}\right) \\
& \leq\left(1-\prod_{i=1}^{n}\left(1-\left(\mu_{\alpha_{i}}^{+}\right)^{\lambda}\right)^{w_{i}}\right)^{1 / \lambda} \\
& \quad\left(1-\left(1-\prod_{i=1}^{n}\left(1-\left(1-v_{\alpha_{i}}^{+}\right)^{\lambda}\right)^{w_{i}}\right)^{1 / \lambda}\right), \\
& \forall \alpha_{1} \in \widetilde{h}_{1}, \alpha_{2} \in \widetilde{h}_{2}, \ldots, \alpha_{n} \in \widetilde{h}_{n},
\end{aligned}
$$

we have

$$
\begin{aligned}
& \prod_{i=1}^{n}\left(\mu_{\alpha_{i}}^{-}\right)^{w_{i}}-\left(1-\prod_{i=1}^{n}\left(1-v_{\alpha_{i}}^{-}\right)^{w_{i}}\right) \\
& =\left(1-\prod_{i=1}^{n}\left(1-\left(\mu_{\alpha_{i}}^{-}\right)^{\lambda}\right)^{w_{i}}\right)^{1 / \lambda} \\
& \quad-\left(1-\left(1-\prod_{i=1}^{n}\left(1-\left(1-v_{\alpha_{i}}^{-}\right)^{\lambda}\right)^{w_{i}}\right)^{1 / \lambda}\right), \\
& \prod_{i=1}^{n}\left(\mu_{\alpha_{i}}^{+}\right)^{w_{i}}-\left(1-\prod_{i=1}^{n}\left(1-v_{\alpha_{i}}^{+}\right)^{w_{i}}\right)
\end{aligned}
$$

$$
\begin{aligned}
= & \left(1-\prod_{i=1}^{n}\left(1-\left(\mu_{\alpha_{i}}^{+}\right)^{\lambda}\right)^{w_{i}}\right)^{1 / \lambda} \\
& -\left(1-\left(1-\prod_{i=1}^{n}\left(1-\left(1-v_{\alpha_{i}}^{+}\right)^{\lambda}\right)^{w_{i}}\right)^{1 / \lambda}\right) .
\end{aligned}
$$

Furthermore, by the conditions that

$$
\prod_{i=1}^{n}\left(\mu_{\alpha_{i}}^{-}\right)^{w_{i}} \leq\left(1-\prod_{i=1}^{n}\left(1-\left(\mu_{\alpha_{i}}^{-}\right)^{\lambda}\right)^{w_{i}}\right)^{1 / \lambda},
$$

$1-\prod_{i=1}^{n}\left(1-v_{\alpha_{i}}^{-}\right)^{w_{i}} \geq 1-\left(1-\prod_{i=1}^{n}\left(1-\left(1-v_{\alpha_{i}}^{-}\right)^{\lambda}\right)^{w_{i}}\right)^{1 / \lambda}$,

$\prod_{i=1}^{n}\left(\mu_{\alpha_{i}}^{+}\right)^{w_{i}} \leq\left(1-\prod_{i=1}^{n}\left(1-\left(\mu_{\alpha_{i}}^{+}\right)^{\lambda}\right)^{w_{i}}\right)^{1 / \lambda}$,

$1-\prod_{i=1}^{n}\left(1-v_{\alpha_{i}}^{+}\right)^{w_{i}} \geq 1-\left(1-\prod_{i=1}^{n}\left(1-\left(1-v_{\alpha_{i}}^{+}\right)^{\lambda}\right)^{w_{i}}\right)^{1 / \lambda}$,

$\forall \alpha_{1} \in \tilde{h}_{1}, \alpha_{2} \in \tilde{h}_{2}, \ldots, \alpha_{n} \in \tilde{h}_{n}$,

(40)

we have

$$
\begin{aligned}
\prod_{i=1}^{n}\left(\mu_{\alpha_{i}}^{-}\right)^{w_{i}} & =\left(1-\prod_{i=1}^{n}\left(1-\left(\mu_{\alpha_{i}}^{-}\right)^{\lambda}\right)^{w_{i}}\right)^{1 / \lambda}, \\
1-\prod_{i=1}^{n}\left(1-\nu_{\alpha_{i}}^{-}\right)^{w_{i}} & =1-\left(1-\prod_{i=1}^{n}\left(1-\left(1-v_{\alpha_{i}}^{-}\right)^{\lambda}\right)^{w_{i}}\right)^{1 / \lambda}, \\
\prod_{i=1}^{n}\left(\mu_{\alpha_{i}}^{+}\right)^{w_{i}} & =\left(1-\prod_{i=1}^{n}\left(1-\left(\mu_{\alpha_{i}}^{+}\right)^{\lambda}\right)^{w_{i}}\right)^{1 / \lambda}, \\
1-\prod_{i=1}^{n}\left(1-v_{\alpha_{i}}^{+}\right)^{w_{i}} & =1-\left(1-\prod_{i=1}^{n}\left(1-\left(1-v_{\alpha_{i}}^{+}\right)^{\lambda}\right)^{w_{i}}\right)^{1 / \lambda} .
\end{aligned}
$$

$$
\begin{aligned}
& h\left(\operatorname{IVIHFWG}\left(\widetilde{h}_{1}, \widetilde{h}_{2}, \ldots, \widetilde{h}_{n}\right)\right) \\
& =\left(\frac{1}{2} \sum_{\alpha_{1} \in \widetilde{h}_{1}, \alpha_{2} \in \widetilde{h}_{2}, \ldots, \alpha_{n} \in \widetilde{h}_{n}}\left[\prod_{i=1}^{n}\left(\mu_{\alpha_{i}}^{-}\right)^{w_{i}}+\left(1-\prod_{i=1}^{n}\left(1-v_{\alpha_{i}}^{-}\right)^{w_{i}}\right)+\prod_{i=1}^{n}\left(\mu_{\alpha_{i}}^{+}\right)^{w_{i}}+\left(1-\prod_{i=1}^{n}\left(1-v_{\alpha_{i}}^{+}\right)^{w_{i}}\right)\right]\right) \\
& \times\left(\#\left(\operatorname{IVIHFWG}\left(\widetilde{h}_{1}, \widetilde{h}_{2}, \ldots, \widetilde{h}_{n}\right)\right)\right)^{-1} \\
& =\left(\frac { 1 } { 2 } \sum _ { \alpha _ { 1 } \in \widetilde { h } _ { 1 } , \alpha _ { 2 } \in \tilde { h } _ { 2 } , \ldots , \alpha _ { n } \in \widetilde { h } _ { n } } \left[\left(1-\prod_{i=1}^{n}\left(1-\left(\mu_{\alpha_{i}}^{-}\right)^{\lambda}\right)^{w_{i}}\right)^{1 / \lambda}+\left(1-\left(1-\prod_{i=1}^{n}\left(1-\left(1-v_{\alpha_{i}}^{-}\right)^{\lambda}\right)^{w_{i}}\right)^{1 / \lambda}\right)\right.\right. \\
& \left.\left.+\left(1-\prod_{i=1}^{n}\left(1-\left(\mu_{\alpha_{i}}^{+}\right)^{\lambda}\right)^{w_{i}}\right)^{1 / \lambda}+\left(1-\left(1-\prod_{i=1}^{n}\left(1-\left(1-v_{\alpha_{i}}^{+}\right)^{\lambda}\right)^{w_{i}}\right)^{1 / \lambda}\right)\right]\right) \\
& \times\left(\#\left(\operatorname{GIVIHFWA}_{\lambda}\left(\widetilde{h}_{1}, \widetilde{h}_{2}, \ldots, \widetilde{h}_{n}\right)\right)\right)^{-1}=h\left(\operatorname{GIVIHFWA}_{\lambda}\left(\widetilde{h}_{1}, \widetilde{h}_{2}, \ldots, \widetilde{h}_{n}\right)\right),
\end{aligned}
$$


which implies that

$$
\operatorname{IVIHFWG}\left(\widetilde{h}_{1}, \widetilde{h}_{2}, \ldots, \widetilde{h}_{n}\right)=\operatorname{GIVIHFWA}_{\lambda}\left(\widetilde{h}_{1}, \widetilde{h}_{2}, \ldots, \widetilde{h}_{n}\right) \text {. }
$$

Based on the previous analysis, we can conclude that (32) always holds.

According to Theorem 20, we can conclude that the values obtained by the IVIHFWG operator are not bigger than the ones obtained by the GIVIHFWA operator for any $\lambda>0$.

If we let $\lambda=1$ in Theorem 20, then we can obtain the following result.

Corollary 21. Suppose that $\widetilde{h}_{i}(i=1,2, \ldots, n)$ is a collection of IVIHFEs, and $w=\left(w_{1}, w_{2}, \ldots, w_{n}\right)^{T}$ is their weight vector with $w_{i} \in[0,1]$ and $\sum_{i=1}^{n} w_{i}=1$; then one has

$$
\operatorname{IVIHFWG}\left(\widetilde{h}_{1}, \tilde{h}_{2}, \ldots, \tilde{h}_{n}\right) \leq \operatorname{IVIHFWA}\left(\widetilde{h}_{1}, \tilde{h}_{2}, \ldots, \tilde{h}_{n}\right) .
$$

Corollary 21 shows that the values obtained by the IVIHFWG operator are not bigger than the ones obtained by the IVIHFWA operator.

Theorem 22. Let $\widetilde{h}_{i}(i=1,2, \ldots, n)$ be a collection of IVIHFEs having the weight vector $w=\left(w_{1}, w_{2}, \ldots, w_{n}\right)^{T}$ such that $w_{i} \in$ $[0,1]$ and $\sum_{i=1}^{n} w_{i}=1, \lambda>0$; then

$$
\operatorname{GIVIHFWG} \bar{\lambda}_{\lambda}\left(\widetilde{h}_{1}, \widetilde{h}_{2}, \ldots, \widetilde{h}_{n}\right) \leq \operatorname{IVIHFWA}\left(\widetilde{h}_{1}, \widetilde{h}_{2}, \ldots, \widetilde{h}_{n}\right) .
$$

Proof. For any $\alpha_{1} \in \widetilde{h}_{1}, \alpha_{2} \in \tilde{h}_{2}, \ldots, \alpha_{n} \in \tilde{h}_{n}$, by Lemma 19 , we have

$$
\begin{aligned}
1 & -\left(1-\prod_{i=1}^{n}\left(1-\left(1-\mu_{\alpha_{i}}^{-}\right)^{\lambda}\right)^{w_{i}}\right)^{1 / \lambda} \\
& \leq 1-\left(1-\sum_{i=1}^{n} w_{i}\left(1-\left(1-\mu_{\alpha_{i}}^{-}\right)^{\lambda}\right)\right)^{1 / \lambda} \\
& =1-\left(\sum_{i=1}^{n} w_{i}\left(1-\mu_{\alpha_{i}}^{-}\right)^{\lambda}\right)^{1 / \lambda} \\
& \leq 1-\left(\prod_{i=1}^{n}\left(1-\mu_{\alpha_{i}}^{-}\right)^{\lambda w_{i}}\right)^{1 / \lambda}
\end{aligned}
$$$$
s\left(\operatorname{GIVIHFWG}_{\lambda}\left(\widetilde{h}_{1}, \tilde{h}_{2}, \ldots, \tilde{h}_{n}\right)\right)
$$

$$
=1-\prod_{i=1}^{n}\left(1-\mu_{\alpha_{i}}^{-}\right)^{w_{i}}
$$$$
1-\left(1-\prod_{i=1}^{n}\left(1-\left(1-\mu_{\alpha_{i}}^{+}\right)^{\lambda}\right)^{w_{i}}\right)^{1 / \lambda}
$$$$
\leq 1-\left(1-\sum_{i=1}^{n} w_{i}\left(1-\left(1-\mu_{\alpha_{i}}^{+}\right)^{\lambda}\right)\right)^{1 / \lambda}
$$$$
=1-\left(\sum_{i=1}^{n} w_{i}\left(1-\mu_{\alpha_{i}}^{+}\right)^{\lambda}\right)^{1 / \lambda}
$$$$
\leq 1-\left(\prod_{i=1}^{n}\left(1-\mu_{\alpha_{i}}^{+}\right)^{\lambda w_{i}}\right)^{1 / \lambda}
$$$$
=1-\prod_{i=1}^{n}\left(1-\mu_{\alpha_{i}}^{+}\right)^{w_{i}}
$$

$$
\begin{aligned}
& \left(1-\prod_{i=1}^{n}\left(1-\left(\nu_{\alpha_{i}}^{-}\right)^{\lambda}\right)^{w_{i}}\right)^{1 / \lambda} \\
& \geq\left(1-\sum_{i=1}^{n} w_{i}\left(1-\left(\nu_{\alpha_{i}}^{-}\right)^{\lambda}\right)\right)^{1 / \lambda} \\
& =\left(\sum_{i=1}^{n} w_{i}\left(\nu_{\alpha_{i}}^{-}\right)^{\lambda}\right)^{1 / \lambda} \\
& \geq\left(\prod_{i=1}^{n}\left(\left(\nu_{\alpha_{i}}^{-}\right)^{\lambda}\right)^{w_{i}}\right)^{1 / \lambda}=\prod_{i=1}^{n}\left(\nu_{\alpha_{i}}^{-}\right)^{w_{i}} \\
& \left(1-\prod_{i=1}^{n}\left(1-\left(\nu_{\alpha_{i}}^{+}\right)^{\lambda}\right)^{w_{i}}\right)^{1 / \lambda} \\
& \geq\left(1-\sum_{i=1}^{n} w_{i}\left(1-\left(v_{\alpha_{i}}^{+}\right)^{\lambda}\right)\right)^{1 / \lambda} \\
& =\left(\sum_{i=1}^{n} w_{i}\left(\nu_{\alpha_{i}}^{+}\right)^{\lambda}\right)^{1 / \lambda} \\
& \geq\left(\prod_{i=1}^{n}\left(\left(\nu_{\alpha_{i}}^{+}\right)^{\lambda}\right)^{w_{i}}\right)^{1 / \lambda}=\prod_{i=1}^{n}\left(\nu_{\alpha_{i}}^{+}\right)^{w_{i}} .
\end{aligned}
$$

$$
\begin{aligned}
=\left(\frac{1}{2} \sum_{\alpha_{1} \in \widetilde{h}_{1}, \alpha_{2} \in \widetilde{h}_{2}, \ldots, \alpha_{n} \in \tilde{h}_{n}}[1\right. & -\left(1-\prod_{i=1}^{n}\left(1-\left(1-\mu_{\alpha_{i}}^{-}\right)^{\lambda}\right)^{w_{i}}\right)^{1 / \lambda}-\left(1-\prod_{i=1}^{n}\left(1-\left(\nu_{\alpha_{i}}^{-}\right)^{\lambda}\right)^{w_{i}}\right)^{1 / \lambda} \\
& \left.\left.+1-\left(1-\prod_{i=1}^{n}\left(1-\left(1-\mu_{\alpha_{i}}^{+}\right)^{\lambda}\right)^{w_{i}}\right)^{1 / \lambda}-\left(1-\prod_{i=1}^{n}\left(1-\left(\nu_{\alpha_{i}}^{+}\right)^{\lambda}\right)^{w_{i}}\right)^{1 / \lambda}\right]\right)
\end{aligned}
$$


Journal of Applied Mathematics

15

$$
\begin{aligned}
& \times\left(\#\left(\operatorname{GIVIHFWG}_{\lambda}\left(\widetilde{h}_{1}, \widetilde{h}_{2}, \ldots, \widetilde{h}_{n}\right)\right)\right)^{-1} \\
& \leq\left(\frac{1}{2} \sum_{\alpha_{1} \in \widetilde{h}_{1}, \alpha_{2} \in \tilde{h}_{2}, \ldots, \alpha_{n} \in \tilde{h}_{n}}\left[1-\prod_{i=1}^{n}\left(1-\mu_{\alpha_{i}}^{-}\right)^{w_{i}}-\prod_{i=1}^{n}\left(v_{\alpha_{i}}^{-}\right)^{w_{i}}+1-\prod_{i=1}^{n}\left(1-\mu_{\alpha_{i}}^{+}\right)^{w_{i}}-\prod_{i=1}^{n}\left(v_{\alpha_{i}}^{+}\right)^{w_{i}}\right]\right) \\
& \times\left(\#\left(\operatorname{IVIHFWA}\left(\widetilde{h}_{1}, \widetilde{h}_{2}, \ldots, \widetilde{h}_{n}\right)\right)\right)^{-1}=s\left(\operatorname{IVIHFWA}\left(\widetilde{h}_{1}, \widetilde{h}_{2}, \ldots, \widetilde{h}_{n}\right)\right) .
\end{aligned}
$$

If $s\left(\operatorname{GIVIHFWG}_{\lambda}\left(\widetilde{h}_{1}, \widetilde{h}_{2}, \ldots, \widetilde{h}_{n}\right)\right)<s\left(\operatorname{IVIHFWA}\left(\widetilde{h}_{1}, \widetilde{h}_{2}\right.\right.$, $\left.\left.\ldots, \widetilde{h}_{n}\right)\right)$, then by Definition 10 , we have

$$
<\operatorname{IVIHFWA}\left(\widetilde{h}_{1}, \widetilde{h}_{2}, \ldots, \widetilde{h}_{n}\right) .
$$

If $s\left(\operatorname{GIVIHFWG}_{\lambda}\left(\widetilde{h}_{1}, \widetilde{h}_{2}, \ldots, \widetilde{h}_{n}\right)\right)=s\left(\operatorname{IVIHFWA}\left(\widetilde{h}_{1}, \widetilde{h}_{2}\right.\right.$,

$$
\begin{aligned}
& \operatorname{GIVIHFWG}_{\lambda}\left(\widetilde{h}_{1}, \widetilde{h}_{2}, \ldots, \widetilde{h}_{n}\right) \\
& \left(\frac { 1 } { 2 } \sum _ { \alpha _ { 1 } \in \tilde { h } _ { 1 } , \alpha _ { 2 } \in \tilde { h } _ { 2 } , \ldots , \alpha _ { n } \in \tilde { h } _ { n } } \left[1-\left(1-\prod_{i=1}^{n}\left(1-\left(1-\mu_{\alpha_{i}}^{-}\right)^{\lambda}\right)^{w_{i}}\right)^{1 / \lambda}-\left(1-\prod_{i=1}^{n}\left(1-\left(\nu_{\alpha_{i}}^{-}\right)^{\lambda}\right)^{w_{i}}\right)^{1 / \lambda}\right.\right. \\
& \left.\left.+1-\left(1-\prod_{i=1}^{n}\left(1-\left(1-\mu_{\alpha_{i}}^{+}\right)^{\lambda}\right)^{w_{i}}\right)^{1 / \lambda}-\left(1-\prod_{i=1}^{n}\left(1-\left(\nu_{\alpha_{i}}^{+}\right)^{\lambda}\right)^{w_{i}}\right)^{1 / \lambda}\right]\right) \\
& \times\left(\#\left(\operatorname{GIVIHFWG}_{\lambda}\left(\widetilde{h}_{1}, \widetilde{h}_{2}, \ldots, \widetilde{h}_{n}\right)\right)\right)^{-1} \\
& =\left(\frac{1}{2} \sum_{\alpha_{1} \in \tilde{h}_{1}, \alpha_{2} \in \tilde{h}_{2}, \ldots, \alpha_{n} \in \tilde{h}_{n}}\left[1-\prod_{i=1}^{n}\left(1-\mu_{\alpha_{i}}^{-}\right)^{w_{i}}-\prod_{i=1}^{n}\left(v_{\alpha_{i}}^{-}\right)^{w_{i}}+1-\prod_{i=1}^{n}\left(1-\mu_{\alpha_{i}}^{+}\right)^{w_{i}}-\prod_{i=1}^{n}\left(v_{\alpha_{i}}^{+}\right)^{w_{i}}\right]\right) \\
& \times\left(\#\left(\operatorname{IVIHFWA}\left(\widetilde{h}_{1}, \widetilde{h}_{2}, \ldots, \widetilde{h}_{n}\right)\right)\right)^{-1} \text {, }
\end{aligned}
$$

then, by the conditions that

$$
\begin{gathered}
1-\left(1-\prod_{i=1}^{n}\left(1-\left(1-\mu_{\alpha_{i}}^{-}\right)^{\lambda}\right)^{w_{i}}\right)^{1 / \lambda} \\
-\left(1-\prod_{i=1}^{n}\left(1-\left(v_{\alpha_{i}}^{-}\right)^{\lambda}\right)^{w_{i}}\right)^{1 / \lambda} \\
\leq 1-\prod_{i=1}^{n}\left(1-\mu_{\alpha_{i}}^{-}\right)^{w_{i}}-\prod_{i=1}^{n}\left(v_{\alpha_{i}}^{-}\right)^{w_{i}}, \\
1-\left(1-\prod_{i=1}^{n}\left(1-\left(1-\mu_{\alpha_{i}}^{+}\right)^{\lambda}\right)^{w_{i}}\right)^{1 / \lambda} \\
-\left(1-\prod_{i=1}^{n}\left(1-\left(v_{\alpha_{i}}^{+}\right)^{\lambda}\right)^{w_{i}}\right)^{1 / \lambda} \\
\leq 1-\prod_{i=1}^{n}\left(1-\mu_{\alpha_{i}}^{+}\right)^{w_{i}}-\prod_{i=1}^{n}\left(v_{\alpha_{i}}^{+}\right)^{w_{i}}, \\
\forall \alpha_{1} \in \tilde{h}_{1}, \alpha_{2} \in \widetilde{h}_{2}, \ldots, \alpha_{n} \in \widetilde{h}_{n},
\end{gathered}
$$

(50) we have

$$
\begin{array}{r}
1-\left(1-\prod_{i=1}^{n}\left(1-\left(1-\mu_{\alpha_{i}}^{-}\right)^{\lambda}\right)^{w_{i}}\right)^{1 / \lambda} \\
-\left(1-\prod_{i=1}^{n}\left(1-\left(v_{\alpha_{i}}^{-}\right)^{\lambda}\right)^{w_{i}}\right)^{1 / \lambda} \\
=1-\prod_{i=1}^{n}\left(1-\mu_{\alpha_{i}}^{-}\right)^{w_{i}}-\prod_{i=1}^{n}\left(\nu_{\alpha_{i}}^{-}\right)^{w_{i}}, \\
1-\left(1-\prod_{i=1}^{n}\left(1-\left(1-\mu_{\alpha_{i}}^{+}\right)^{\lambda}\right)^{w_{i}}\right)^{1 / \lambda} \\
-\left(1-\prod_{i=1}^{n}\left(1-\left(v_{\alpha_{i}}^{+}\right)^{\lambda}\right)^{w_{i}}\right)^{1 / \lambda} \\
=1-\prod_{i=1}^{n}\left(1-\mu_{\alpha_{i}}^{+}\right)^{w_{i}}-\prod_{i=1}^{n}\left(\nu_{\alpha_{i}}^{+}\right)^{w_{i} .}
\end{array}
$$

(51) 
16

Journal of Applied Mathematics

Furthermore, by the conditions that

$$
\begin{gathered}
1-\left(1-\prod_{i=1}^{n}\left(1-\left(1-\mu_{\alpha_{i}}^{-}\right)^{\lambda}\right)^{w_{i}}\right)^{1 / \lambda} \leq 1-\prod_{i=1}^{n}\left(1-\mu_{\alpha_{i}}^{-}\right)^{w_{i}}, \\
1-\left(1-\prod_{i=1}^{n}\left(1-\left(1-\mu_{\alpha_{i}}^{+}\right)^{\lambda}\right)^{w_{i}}\right)^{1 / \lambda} \leq 1-\prod_{i=1}^{n}\left(1-\mu_{\alpha_{i}}^{+}\right)^{w_{i}}, \\
\left(1-\prod_{i=1}^{n}\left(1-\left(\nu_{\alpha_{i}}^{-}\right)^{\lambda}\right)^{w_{i}}\right)^{1 / \lambda} \geq \prod_{i=1}^{n}\left(\nu_{\alpha_{i}}^{-}\right)^{w_{i}}, \\
\left(1-\prod_{i=1}^{n}\left(1-\left(\nu_{\alpha_{i}}^{+}\right)^{\lambda}\right)^{w_{i}}\right)^{1 / \lambda} \geq \prod_{i=1}^{n}\left(v_{\alpha_{i}}^{+}\right)^{w_{i}}, \\
\forall \alpha_{1} \in \tilde{h}_{1}, \alpha_{2} \in \tilde{h}_{2}, \ldots, \alpha_{n} \in \tilde{h}_{n},
\end{gathered}
$$

we have

$$
\begin{gathered}
1-\left(1-\prod_{i=1}^{n}\left(1-\left(1-\mu_{\alpha_{i}}^{-}\right)^{\lambda}\right)^{w_{i}}\right)^{1 / \lambda}=1-\prod_{i=1}^{n}\left(1-\mu_{\alpha_{i}}^{-}\right)^{w_{i}}, \\
1-\left(1-\prod_{i=1}^{n}\left(1-\left(1-\mu_{\alpha_{i}}^{+}\right)^{\lambda}\right)^{w_{i}}\right)^{1 / \lambda}=1-\prod_{i=1}^{n}\left(1-\mu_{\alpha_{i}}^{+}\right)^{w_{i}}, \\
\left(1-\prod_{i=1}^{n}\left(1-\left(\nu_{\alpha_{i}}^{-}\right)^{\lambda}\right)^{w_{i}}\right)^{1 / \lambda}=\prod_{i=1}^{n}\left(\nu_{\alpha_{i}}^{-}\right)^{w_{i}}, \\
\left(1-\prod_{i=1}^{n}\left(1-\left(\nu_{\alpha_{i}}^{+}\right)^{\lambda}\right)^{w_{i}}\right)^{1 / \lambda}=\prod_{i=1}^{n}\left(\nu_{\alpha_{i}}^{+}\right)^{w_{i}} .
\end{gathered}
$$

Thus,

$$
\begin{aligned}
& h\left(\operatorname{GIVIHFWG}_{\lambda}\left(\widetilde{h}_{1}, \widetilde{h}_{2}, \ldots, \widetilde{h}_{n}\right)\right) \\
& =\left(\frac { 1 } { 2 } \sum _ { \alpha _ { 1 } \in \tilde { h } _ { 1 } , \alpha _ { 2 } \in \tilde { h } _ { 2 } , \ldots , \alpha _ { n } \in \tilde { h } _ { n } } \left[1-\left(1-\prod_{i=1}^{n}\left(1-\left(1-\mu_{\alpha_{i}}^{-}\right)^{\lambda}\right)^{w_{i}}\right)^{1 / \lambda}+\left(1-\prod_{i=1}^{n}\left(1-\left(\nu_{\alpha_{i}}^{-}\right)^{\lambda}\right)^{w_{i}}\right)^{1 / \lambda}\right.\right. \\
& \left.\left.+1-\left(1-\prod_{i=1}^{n}\left(1-\left(1-\mu_{\alpha_{i}}^{+}\right)^{\lambda}\right)^{w_{i}}\right)^{1 / \lambda}+\left(1-\prod_{i=1}^{n}\left(1-\left(v_{\alpha_{i}}^{+}\right)^{\lambda}\right)^{w_{i}}\right)^{1 / \lambda}\right]\right) \\
& \times\left(\#\left(\operatorname{GIVIHFWG}_{\lambda}\left(\widetilde{h}_{1}, \widetilde{h}_{2}, \ldots, \widetilde{h}_{n}\right)\right)\right)^{-1} \\
& =\left(\frac{1}{2} \sum_{\alpha_{1} \in \tilde{h}_{1}, \alpha_{2} \in \widetilde{h}_{2}, \ldots, \alpha_{n} \in \tilde{h}_{n}}\left[1-\prod_{i=1}^{n}\left(1-\mu_{\alpha_{i}}^{-}\right)^{w_{i}}+\prod_{i=1}^{n}\left(\nu_{\alpha_{i}}^{-}\right)^{w_{i}}+1-\prod_{i=1}^{n}\left(1-\mu_{\alpha_{i}}^{+}\right)^{w_{i}}+\prod_{i=1}^{n}\left(\nu_{\alpha_{i}}^{+}\right)^{w_{i}}\right]\right) \\
& \times\left(\#\left(\operatorname{IVIHFWA}\left(\widetilde{h}_{1}, \widetilde{h}_{2}, \ldots, \widetilde{h}_{n}\right)\right)\right)^{-1}=h\left(\operatorname{IVIHFWA}\left(\widetilde{h}_{1}, \widetilde{h}_{2}, \ldots, \widetilde{h}_{n}\right)\right),
\end{aligned}
$$

which implies that

$$
\operatorname{GIVIHFWG}{ }_{\lambda}\left(\widetilde{h}_{1}, \widetilde{h}_{2}, \ldots, \widetilde{h}_{n}\right)=\operatorname{IVIHFWA}\left(\widetilde{h}_{1}, \widetilde{h}_{2}, \ldots, \widetilde{h}_{n}\right)
$$

Based on the previous analysis, we can conclude that (56) always holds.

Theorem 22 tells us that the values obtained by the GIVIHFWG operator are not bigger than the ones obtained by the IVIHFWA operator for any $\lambda>0$.

Theorem 23. Let $\widetilde{h}_{i}(i=1,2, \ldots, n)$ be a collection of IVIHFEs having the weight vector $w=\left(w_{1}, w_{2}, \ldots, w_{n}\right)^{T}$ such that $w_{i} \in$ $[0,1]$ and $\sum_{i=1}^{n} w_{i}=1, \lambda>0$; then

$$
\begin{aligned}
& \operatorname{GIVIHFWG}_{\lambda}\left(\widetilde{h}_{1}, \widetilde{h}_{2}, \ldots, \widetilde{h}_{n}\right) \\
& \quad \leq \operatorname{GIVIHFWA}_{\lambda}\left(\widetilde{h}_{1}, \widetilde{h}_{2}, \ldots, \widetilde{h}_{n}\right) .
\end{aligned}
$$

Proof. According to the proof of Theorem 3.10 in [13], we can obtain

$$
\begin{aligned}
& \left(1-\prod_{i=1}^{n}\left(1-\left(\mu_{\alpha_{i}}^{-}\right)^{\lambda}\right)^{w_{i}}\right)^{1 / \lambda} \\
& \geq 1-\left(1-\prod_{i=1}^{n}\left(1-\left(1-\mu_{\alpha_{i}}^{-}\right)^{\lambda}\right)^{w_{i}}\right)^{1 / \lambda}, \\
& \left(1-\prod_{i=1}^{n}\left(1-\left(\mu_{\alpha_{i}}^{+}\right)^{\lambda}\right)^{w_{i}}\right)^{1 / \lambda} \\
& \geq 1-\left(1-\prod_{i=1}^{n}\left(1-\left(1-\mu_{\alpha_{i}}^{+}\right)^{\lambda}\right)^{w_{i}}\right)^{1 / \lambda}, \\
& 1-\left(1-\prod_{i=1}^{n}\left(1-\left(1-\nu_{\alpha_{i}}^{-}\right)^{\lambda}\right)^{w_{i}}\right)^{1 / \lambda}
\end{aligned}
$$


Journal of Applied Mathematics

17

$$
\begin{array}{ll}
\leq\left(1-\prod_{i=1}^{n}\left(1-\left(v_{\alpha_{i}}^{-}\right)^{\lambda}\right)^{w_{i}}\right)^{1 / \lambda}, & \leq\left(1-\prod_{i=1}^{n}\left(1-\left(v_{\alpha_{i}}^{+}\right)^{\lambda}\right)^{w_{i}}\right)^{1 / \lambda} . \\
1-\left(1-\prod_{i=1}^{n}\left(1-\left(1-v_{\alpha_{i}}^{+}\right)^{\lambda}\right)^{w_{i}}\right)^{1 / \lambda} & \text { By Definition 10, we have }
\end{array}
$$

By Definition 10, we have

$$
\begin{aligned}
& s\left(\operatorname{GIVIHFWG}_{\lambda}\left(\widetilde{h}_{1}, \widetilde{h}_{2}, \ldots, \widetilde{h}_{n}\right)\right) \\
& =\left(\frac { 1 } { 2 } \sum _ { \alpha _ { 1 } \in \widetilde { h } _ { 1 } , \alpha _ { 2 } \in \widetilde { h } _ { 2 } , \ldots , \alpha _ { n } \in \widetilde { h } _ { n } } \left[1-\left(1-\prod_{i=1}^{n}\left(1-\left(1-\mu_{\alpha_{i}}^{-}\right)^{\lambda}\right)^{w_{i}}\right)^{1 / \lambda}-\left(1-\prod_{i=1}^{n}\left(1-\left(v_{\alpha_{i}}^{-}\right)^{\lambda}\right)^{w_{i}}\right)^{1 / \lambda}\right.\right. \\
& \left.\left.+1-\left(1-\prod_{i=1}^{n}\left(1-\left(1-\mu_{\alpha_{i}}^{+}\right)^{\lambda}\right)^{w_{i}}\right)^{1 / \lambda}-\left(1-\prod_{i=1}^{n}\left(1-\left(\nu_{\alpha_{i}}^{+}\right)^{\lambda}\right)^{w_{i}}\right)^{1 / \lambda}\right]\right) \\
& \times\left(\#\left(\operatorname{GIVIHFWG}_{\lambda}\left(\widetilde{h}_{1}, \tilde{h}_{2}, \ldots, \widetilde{h}_{n}\right)\right)\right)^{-1} \\
& \leq\left(\frac { 1 } { 2 } \sum _ { \alpha _ { 1 } \in \tilde { h } _ { 1 } , \alpha _ { 2 } \in \tilde { h } _ { 2 } , \ldots , \alpha _ { n } \in \widetilde { h } _ { n } } \left[\left(1-\prod_{i=1}^{n}\left(1-\left(\mu_{\alpha_{i}}^{-}\right)^{\lambda}\right)^{w_{i}}\right)^{1 / \lambda}-\left(1-\left(1-\prod_{i=1}^{n}\left(1-\left(1-v_{\alpha_{i}}^{-}\right)^{\lambda}\right)^{w_{i}}\right)^{1 / \lambda}\right)\right.\right. \\
& \left.\left.+\left(1-\prod_{i=1}^{n}\left(1-\left(\mu_{\alpha_{i}}^{+}\right)^{\lambda}\right)^{w_{i}}\right)^{1 / \lambda}-\left(1-\left(1-\prod_{i=1}^{n}\left(1-\left(1-v_{\alpha_{i}}^{+}\right)^{\lambda}\right)^{w_{i}}\right)^{1 / \lambda}\right)\right]\right) \\
& \times\left(\#\left(\operatorname{GIVIHFWA}_{\lambda}\left(\tilde{h}_{1}, \tilde{h}_{2}, \ldots, \tilde{h}_{n}\right)\right)\right)^{-1}=s\left(\operatorname{GIVIHFWA}_{\lambda}\left(\widetilde{h}_{1}, \tilde{h}_{2}, \ldots, \widetilde{h}_{n}\right)\right) .
\end{aligned}
$$

If $s\left(\operatorname{GIVIHFWG}_{\lambda}\left(\widetilde{h}_{1}, \widetilde{h}_{2}, \ldots, \widetilde{h}_{n}\right)\right)<s\left(\operatorname{GIVIHFWA}_{\lambda}\left(\widetilde{h}_{1}, \widetilde{h}_{2}\right.\right.$, $\left.\left.\ldots, \widetilde{h}_{n}\right)\right)$, then by Definition 10 , we have

$$
<\operatorname{GIVIHFWA}_{\lambda}\left(\widetilde{h}_{1}, \tilde{h}_{2}, \ldots, \tilde{h}_{n}\right)
$$

If $s\left(\operatorname{GIVIHFWG}_{\lambda}\left(\tilde{h}_{1}, \tilde{h}_{2}, \ldots, \tilde{h}_{n}\right)\right)=s\left(\operatorname{GIVIHFWA}_{\lambda}\left(\tilde{h}_{1}, \tilde{h}_{2}\right.\right.$, $\left.\left.\ldots, \widetilde{h}_{n}\right)\right)$, that is,

$$
\operatorname{GIVIHFWG}_{\lambda}\left(\widetilde{h}_{1}, \tilde{h}_{2}, \ldots, \tilde{h}_{n}\right)
$$

$$
\begin{aligned}
& \left(\frac { 1 } { 2 } \sum _ { \alpha _ { 1 } \in \tilde { h } _ { 1 } , \alpha _ { 2 } \in \tilde { h } _ { 2 } , \ldots , \alpha _ { n } \in \tilde { h } _ { n } } \left[1-\left(1-\prod_{i=1}^{n}\left(1-\left(1-\mu_{\alpha_{i}}^{-}\right)^{\lambda}\right)^{w_{i}}\right)^{1 / \lambda}-\left(1-\prod_{i=1}^{n}\left(1-\left(v_{\alpha_{i}}^{-}\right)^{\lambda}\right)^{w_{i}}\right)^{1 / \lambda}\right.\right. \\
& \left.\left.+1-\left(1-\prod_{i=1}^{n}\left(1-\left(1-\mu_{\alpha_{i}}^{+}\right)^{\lambda}\right)^{w_{i}}\right)^{1 / \lambda}-\left(1-\prod_{i=1}^{n}\left(1-\left(v_{\alpha_{i}}^{+}\right)^{\lambda}\right)^{w_{i}}\right)^{1 / \lambda}\right]\right) \\
& \times\left(\#\left(\operatorname{GIVIHFWG}_{\lambda}\left(\tilde{h}_{1}, \tilde{h}_{2}, \ldots, \tilde{h}_{n}\right)\right)\right)^{-1} \\
& =\left(\frac { 1 } { 2 } \sum _ { \alpha _ { 1 } \in \widetilde { h } _ { 1 } , \alpha _ { 2 } \in \tilde { h } _ { 2 } , \ldots , \alpha _ { n } \in \tilde { h } _ { n } } \left[\left(1-\prod_{i=1}^{n}\left(1-\left(\mu_{\alpha_{i}}^{-}\right)^{\lambda}\right)^{w_{i}}\right)^{1 / \lambda}-\left(1-\left(1-\prod_{i=1}^{n}\left(1-\left(1-v_{\alpha_{i}}^{-}\right)^{\lambda}\right)^{w_{i}}\right)^{1 / \lambda}\right)\right.\right. \\
& \left.\left.+\left(1-\prod_{i=1}^{n}\left(1-\left(\mu_{\alpha_{i}}^{+}\right)^{\lambda}\right)^{w_{i}}\right)^{1 / \lambda}-\left(1-\left(1-\prod_{i=1}^{n}\left(1-\left(1-v_{\alpha_{i}}^{+}\right)^{\lambda}\right)^{w_{i}}\right)^{1 / \lambda}\right)\right]\right) \\
& \times\left(\#\left(\operatorname{GIVIHFWA}{ }_{\lambda}\left(\widetilde{h}_{1}, \widetilde{h}_{2}, \ldots, \widetilde{h}_{n}\right)\right)\right)^{-1}
\end{aligned}
$$


18

Journal of Applied Mathematics

then, by the conditions that

$$
\begin{aligned}
& 1-\left(1-\prod_{i=1}^{n}\left(1-\left(1-\mu_{\alpha_{i}}^{-}\right)^{\lambda}\right)^{w_{i}}\right)^{1 / \lambda} \\
&-\left(1-\prod_{i=1}^{n}\left(1-\left(v_{\alpha_{i}}^{-}\right)^{\lambda}\right)^{w_{i}}\right)^{1 / \lambda} \\
& \leq\left(1-\prod_{i=1}^{n}\left(1-\left(\mu_{\alpha_{i}}^{-}\right)^{\lambda}\right)^{w_{i}}\right)^{1 / \lambda} \\
&-\left(1-\left(1-\prod_{i=1}^{n}\left(1-\left(1-v_{\alpha_{i}}^{-}\right)^{\lambda}\right)^{w_{i}}\right)^{1 / \lambda}\right) \\
& 1-\left(1-\prod_{i=1}^{n}\left(1-\left(1-\mu_{\alpha_{i}}^{+}\right)^{\lambda}\right)^{w_{i}}\right)^{1 / \lambda} \\
&-\left(1-\prod_{i=1}^{n}\left(1-\left(v_{\alpha_{i}}^{+}\right)^{\lambda}\right)^{w_{i}}\right)^{1 / \lambda} \\
& \leq\left(1-\prod_{i=1}^{n}\left(1-\left(\mu_{\alpha_{i}}^{+}\right)^{\lambda}\right)^{w_{i}}\right)^{1 / \lambda} \\
&-\left(1-\left(1-\prod_{i=1}^{n}\left(1-\left(1-v_{\alpha_{i}}^{+}\right)^{\lambda}\right)^{w_{i}}\right)^{1 / \lambda}\right) \\
& \forall \alpha_{1} \in \widetilde{h}_{1}, \alpha_{2} \in \widetilde{h}_{2}, \ldots, \alpha_{n} \in \widetilde{h}_{n},
\end{aligned}
$$

we have

$$
\begin{aligned}
1- & \left(1-\prod_{i=1}^{n}\left(1-\left(1-\mu_{\alpha_{i}}^{-}\right)^{\lambda}\right)^{w_{i}}\right)^{1 / \lambda} \\
& -\left(1-\prod_{i=1}^{n}\left(1-\left(v_{\alpha_{i}}^{-}\right)^{\lambda}\right)^{w_{i}}\right)^{1 / \lambda} \\
= & \left(1-\prod_{i=1}^{n}\left(1-\left(\mu_{\alpha_{i}}^{-}\right)^{\lambda}\right)^{w_{i}}\right)^{1 / \lambda} \\
& -\left(1-\left(1-\prod_{i=1}^{n}\left(1-\left(1-v_{\alpha_{i}}^{-}\right)^{\lambda}\right)^{w_{i}}\right)^{1 / \lambda}\right), \\
1- & \left(1-\prod_{i=1}^{n}\left(1-\left(1-\mu_{\alpha_{i}}^{+}\right)^{\lambda}\right)^{w_{i}}\right)^{1 / \lambda} \\
& -\left(1-\prod_{i=1}^{n}\left(1-\left(v_{\alpha_{i}}^{+}\right)^{\lambda}\right)^{w_{i}}\right)^{1 / \lambda} \\
= & \left(1-\prod_{i=1}^{n}\left(1-\left(\mu_{\alpha_{i}}^{+}\right)^{\lambda}\right)^{w_{i}}\right)^{1 / \lambda} \\
& -\left(1-\left(1-\prod_{i=1}^{n}\left(1-\left(1-v_{\alpha_{i}}^{+}\right)^{\lambda}\right)^{w_{i}}\right)^{1 / \lambda}\right) .
\end{aligned}
$$

(61)

Furthermore, by the conditions that

$$
\begin{aligned}
& \left(1-\prod_{i=1}^{n}\left(1-\left(\mu_{\alpha_{i}}^{-}\right)^{\lambda}\right)^{w_{i}}\right)^{1 / \lambda} \\
& \geq 1-\left(1-\prod_{i=1}^{n}\left(1-\left(1-\mu_{\alpha_{i}}^{-}\right)^{\lambda}\right)^{w_{i}}\right)^{1 / \lambda}, \\
& \left(1-\prod_{i=1}^{n}\left(1-\left(\mu_{\alpha_{i}}^{+}\right)^{\lambda}\right)^{w_{i}}\right)^{1 / \lambda} \\
& \geq 1-\left(1-\prod_{i=1}^{n}\left(1-\left(1-\mu_{\alpha_{i}}^{+}\right)^{\lambda}\right)^{w_{i}}\right)^{1 / \lambda}, \\
& 1-\left(1-\prod_{i=1}^{n}\left(1-\left(1-v_{\alpha_{i}}^{-}\right)^{\lambda}\right)^{w_{i}}\right)^{1 / \lambda} \\
& \leq\left(1-\prod_{i=1}^{n}\left(1-\left(v_{\alpha_{i}}^{-}\right)^{\lambda}\right)^{w_{i}}\right)^{1 / \lambda}, \\
& 1-\left(1-\prod_{i=1}^{n}\left(1-\left(1-v_{\alpha_{i}}^{+}\right)^{\lambda}\right)^{w_{i}}\right)^{1 / \lambda} \\
& \leq\left(1-\prod_{i=1}^{n}\left(1-\left(v_{\alpha_{i}}^{+}\right)^{\lambda}\right)^{w_{i}}\right)^{1 / \lambda}, \\
& \forall \alpha_{1} \in \widetilde{h}_{1}, \alpha_{2} \in \widetilde{h}_{2}, \ldots, \alpha_{n} \in \widetilde{h}_{n},
\end{aligned}
$$

we have

$$
\begin{aligned}
& \left(1-\prod_{i=1}^{n}\left(1-\left(\mu_{\alpha_{i}}^{-}\right)^{\lambda}\right)^{w_{i}}\right)^{1 / \lambda} \\
& =1-\left(1-\prod_{i=1}^{n}\left(1-\left(1-\mu_{\alpha_{i}}^{-}\right)^{\lambda}\right)^{w_{i}}\right)^{1 / \lambda}, \\
& \left(1-\prod_{i=1}^{n}\left(1-\left(\mu_{\alpha_{i}}^{+}\right)^{\lambda}\right)^{w_{i}}\right)^{1 / \lambda} \\
& =1-\left(1-\prod_{i=1}^{n}\left(1-\left(1-\mu_{\alpha_{i}}^{+}\right)^{\lambda}\right)^{w_{i}}\right)^{1 / \lambda}, \\
& 1-\left(1-\prod_{i=1}^{n}\left(1-\left(1-v_{\alpha_{i}}^{-}\right)^{\lambda}\right)^{w_{i}}\right)^{1 / \lambda} \\
& =\left(1-\prod_{i=1}^{n}\left(1-\left(v_{\alpha_{i}}^{-}\right)^{\lambda}\right)^{w_{i}}\right)^{1 / \lambda}, \\
& 1-\left(1-\prod_{i=1}^{n}\left(1-\left(1-v_{\alpha_{i}}^{+}\right)^{\lambda}\right)^{w_{i}}\right)^{1 / \lambda} \\
& =\left(1-\prod_{i=1}^{n}\left(1-\left(v_{\alpha_{i}}^{+}\right)^{\lambda}\right)^{w_{i}}\right)^{1 / \lambda} .
\end{aligned}
$$

(64) 
Thus,

$$
\begin{aligned}
& h\left(\operatorname{GIVIHFWG}_{\lambda}\left(\widetilde{h}_{1}, \widetilde{h}_{2}, \ldots, \widetilde{h}_{n}\right)\right) \\
& =\left(\frac { 1 } { 2 } \sum _ { \alpha _ { 1 } \in \tilde { h } _ { 1 } , \alpha _ { 2 } \in \tilde { h } _ { 2 } , \ldots , \alpha _ { n } \in \widetilde { h } _ { n } } \left[1-\left(1-\prod_{i=1}^{n}\left(1-\left(1-\mu_{\alpha_{i}}^{-}\right)^{\lambda}\right)^{w_{i}}\right)^{1 / \lambda}+\left(1-\prod_{i=1}^{n}\left(1-\left(v_{\alpha_{i}}^{-}\right)^{\lambda}\right)^{w_{i}}\right)^{1 / \lambda}\right.\right. \\
& \left.\left.+1-\left(1-\prod_{i=1}^{n}\left(1-\left(1-\mu_{\alpha_{i}}^{+}\right)^{\lambda}\right)^{w_{i}}\right)^{1 / \lambda}+\left(1-\prod_{i=1}^{n}\left(1-\left(v_{\alpha_{i}}^{+}\right)^{\lambda}\right)^{w_{i}}\right)^{1 / \lambda}\right]\right) \\
& \times\left(\#\left(\operatorname{GIVIHFWG}_{\lambda}\left(\widetilde{h}_{1}, \widetilde{h}_{2}, \ldots, \widetilde{h}_{n}\right)\right)\right)^{-1} \\
& =\left(\frac { 1 } { 2 } \sum _ { \alpha _ { 1 } \in \tilde { h } _ { 1 } , \alpha _ { 2 } \in \tilde { h } _ { 2 } , \ldots , \alpha _ { n } \in \tilde { h } _ { n } } \left[\left(1-\prod_{i=1}^{n}\left(1-\left(\mu_{\alpha_{i}}^{-}\right)^{\lambda}\right)^{w_{i}}\right)^{1 / \lambda}+\left(1-\left(1-\prod_{i=1}^{n}\left(1-\left(1-v_{\alpha_{i}}^{-}\right)^{\lambda}\right)^{w_{i}}\right)^{1 / \lambda}\right)\right.\right. \\
& \left.\left.+\left(1-\prod_{i=1}^{n}\left(1-\left(\mu_{\alpha_{i}}^{+}\right)^{\lambda}\right)^{w_{i}}\right)^{1 / \lambda}+\left(1-\left(1-\prod_{i=1}^{n}\left(1-\left(1-\nu_{\alpha_{i}}^{+}\right)^{\lambda}\right)^{w_{i}}\right)^{1 / \lambda}\right)\right]\right) \\
& \times\left(\#\left(\operatorname{GIVIHFWA}_{\lambda}\left(\widetilde{h}_{1}, \widetilde{h}_{2}, \ldots, \widetilde{h}_{n}\right)\right)\right)^{-1}=h\left(\operatorname{GIVIHFWA}_{\lambda}\left(\widetilde{h}_{1}, \widetilde{h}_{2}, \ldots, \widetilde{h}_{n}\right)\right) \text {, }
\end{aligned}
$$

which implies that

$$
\begin{aligned}
& \operatorname{GIVIHFWG}_{\lambda}\left(\widetilde{h}_{1}, \widetilde{h}_{2}, \ldots, \widetilde{h}_{n}\right) \\
& \quad=\operatorname{GIVIHFWA}_{\lambda}\left(\widetilde{h}_{1}, \widetilde{h}_{2}, \ldots, \widetilde{h}_{n}\right) .
\end{aligned}
$$

Based on the previous analysis, we can conclude that (56) always holds.

Theorem 23 shows us that, for the same value of the parameter $\lambda>0$ and the same aggregation values, the values obtained by the GIVIHFWA operator are always greater than the ones obtained by the GIVIHFWG operator.

We now look at some special cases of the IVIHFWA, IVIHFWG, GIVIHFWA, and GIVIHFWG operators obtained by using different choices of the input arguments and the weight vector.

(1) If $\widetilde{h}_{i}(i=1,2, \ldots, n)$ is a collection of HFEs, then the IVIHFWA operator reduces to the hesitant fuzzy weighted averaging (HFWA) operator [9], the IVIHFWG operator reduces to the hesitant fuzzy weighted geometric (HFWG) operator [9], the GIVIHFWA operator reduces to the generalized hesitant fuzzy weighted averaging (GHFWA) operator [9], and the GIVIHFWG operator reduces to the generalized hesitant fuzzy weighted geometric (GHFWG) operator [9].

(2) If $\widetilde{h}_{i}(i=1,2, \ldots, n)$ is a collection of IVHFEs, then the IVIHFWA operator reduces to the interval-valued hesitant fuzzy weighted averaging (IVHFWA) operator [6], the IVIHFWG operator reduces to the interval-valued hesitant fuzzy weighted geometric (IVHFWG) operator [6], the GIVIHFWA operator reduces to the generalized interval-valued hesitant fuzzy weighted averaging (GIVHFWA) operator [6], and the GIVIHFWG operator reduces to the generalized interval-valued hesitant fuzzy weighted geometric (GIVHFWG) operator [6].

(3) If $\widetilde{h}_{i}(i=1,2, \ldots, n)$ are a collection of IHFEs, then the IVIHFWA operator reduces to the intuitionistic hesitant fuzzy weighted averaging (IHFWA) operator:

$$
\begin{aligned}
& \operatorname{IHFWA}\left(\widetilde{h}_{1}, \widetilde{h}_{2}, \ldots, \widetilde{h}_{n}\right) \\
& =\bigoplus_{i=1}^{n}\left(w_{i} \widetilde{h}_{i}\right) \\
& =\left\{\left(1-\prod_{i=1}^{n}\left(1-\mu_{\alpha_{i}}\right)^{w_{i}}, \prod_{i=1}^{n}\left(v_{\alpha_{i}}\right)^{w_{i}}\right) \mid\right. \\
& \left.\alpha_{1} \in \widetilde{h}_{1}, \alpha_{2} \in \widetilde{h}_{2}, \ldots, \alpha_{n} \in \widetilde{h}_{n}\right\} .
\end{aligned}
$$

If $\widetilde{h}_{i}(i=1,2, \ldots, n)$ are a collection of IHFEs, then the IVIHFWG operator reduces to the intuitionistic hesitant fuzzy weighted geometric (IHFWG) operator:

$$
\begin{aligned}
& \operatorname{IHFWG}\left(\widetilde{h}_{1}, \widetilde{h}_{2}, \ldots, \widetilde{h}_{n}\right) \\
& =\bigotimes_{i=1}^{n}\left(\widetilde{h}_{i}^{w_{i}}\right) \\
& =\left\{\left(\prod_{i=1}^{n}\left(\mu_{\alpha_{i}}\right)^{w_{i}}, 1-\prod_{i=1}^{n}\left(1-v_{\alpha_{i}}\right)^{w_{i}}\right) \mid\right. \\
& \left.\alpha_{1} \in \widetilde{h}_{1}, \alpha_{2} \in \widetilde{h}_{2}, \ldots, \alpha_{n} \in \widetilde{h}_{n}\right\} .
\end{aligned}
$$


If $\widetilde{h}_{i}(i=1,2, \ldots, n)$ are a collection of IHFEs, then the GIVIHFWA operator reduces to the generalized intuitionistic hesitant fuzzy weighted averaging (GIHFWA) operator:

$$
\begin{gathered}
\operatorname{GIHFWA}\left(\widetilde{h}_{1}, \tilde{h}_{2}, \ldots, \widetilde{h}_{n}\right) \\
=\left(\bigoplus_{i=1}^{n}\left(w_{i} \widetilde{h}_{i}^{\lambda}\right)\right)^{1 / \lambda} \\
=\left\{\left(1-\left(1-\prod_{i=1}^{n}\left(1-\left(1-\mu_{\alpha_{i}}\right)^{\lambda}\right)^{w_{i}}\right)^{1 / \lambda},\right.\right. \\
\left.\left(1-\prod_{i=1}^{n}\left(1-\left(v_{\alpha_{i}}\right)^{\lambda}\right)^{w_{i}}\right)^{1 / \lambda}\right) \mid \\
\left.\alpha_{1} \in \widetilde{h}_{1}, \alpha_{2} \in \widetilde{h}_{2}, \ldots, \alpha_{n} \in \widetilde{h}_{n}\right\} .
\end{gathered}
$$

If $\widetilde{h}_{i}(i=1,2, \ldots, n)$ are a collection of IHFEs, then the GIVIHFWG operator reduces to the generalized intuitionistic hesitant fuzzy weighted geometric (GIHFWG) operator:

$$
\begin{gathered}
\operatorname{GIHFWG}\left(\widetilde{h}_{1}, \widetilde{h}_{2}, \ldots, \widetilde{h}_{n}\right) \\
=\frac{1}{\lambda}\left(\bigotimes_{i=1}^{n}\left(\lambda \widetilde{h}_{i}\right)^{w_{i}}\right) \\
=\left\{\left(1-\left(1-\prod_{i=1}^{n}\left(1-\left(1-\mu_{\alpha_{i}}\right)^{\lambda}\right)^{w_{i}}\right)^{1 / \lambda},\right.\right. \\
\left.\left(1-\prod_{i=1}^{n}\left(1-\left(\nu_{\alpha_{i}}\right)^{\lambda}\right)^{w_{i}}\right)^{1 / \lambda}\right) \mid \\
\left.\alpha_{1} \in \widetilde{h}_{1}, \alpha_{2} \in \tilde{h}_{2}, \ldots, \alpha_{n} \in \widetilde{h}_{n}\right\} .
\end{gathered}
$$

\subsection{The IVIHFOWA, IVIHFOWG, GIVIHFOWA, and} GIVIHFOWG Operators

Definition 24. Let $\widetilde{h}_{i}(i=1,2, \ldots, n)$ be a collection of IVIHFEs, $\widetilde{h}_{\sigma(i)}$ the $i$ th largest one of them, and $\omega=$ $\left(\omega_{1}, \omega_{2}, \ldots, \omega_{n}\right)^{T}$ the aggregation-associated vector such that $\omega_{i} \in[0,1]$ and $\sum_{i=1}^{n} \omega_{i}=1$, then the following hold.

(1) An interval-valued intuitionistic hesitant fuzzy ordered weighted averaging (IVIHFOWA) operator is a mapping $\widetilde{H}^{n} \rightarrow \widetilde{H}$, where

$$
\operatorname{IVIHFOWA}\left(\widetilde{h}_{1}, \widetilde{h}_{2}, \ldots, \widetilde{h}_{n}\right)=\bigoplus_{i=1}^{n}\left(\omega_{i} \widetilde{h}_{\sigma(i)}\right)
$$

(2) An interval-valued intuitionistic hesitant fuzzy ordered weighted geometric (IVIHFOWG) operator is a mapping $\widetilde{H}^{n} \rightarrow \widetilde{H}$, where

$$
\operatorname{IVIHFOWG}\left(\widetilde{h}_{1}, \widetilde{h}_{2}, \ldots, \widetilde{h}_{n}\right)=\bigotimes_{i=1}^{n}\left(\widetilde{h}_{\sigma(i)}^{\omega_{i}}\right) \text {. }
$$

(3) A generalized interval-valued intuitionistic hesitant fuzzy ordered weighted averaging (GIVIHFOWA) operator is a mapping $\widetilde{H}^{n} \rightarrow \widetilde{H}$, where

$$
\operatorname{GIVIHFOWA}_{\lambda}\left(\widetilde{h}_{1}, \widetilde{h}_{2}, \ldots, \widetilde{h}_{n}\right)=\left(\bigoplus_{i=1}^{n}\left(\omega_{i} \widetilde{h}_{\sigma(i)}^{\lambda}\right)\right)^{1 / \lambda}
$$

with $\lambda>0$.

(4) A generalized interval-valued intuitionistic hesitant fuzzy ordered weighted geometric (GIVIHFOWG) operator is a mapping $\widetilde{H}^{n} \rightarrow \widetilde{H}$, where

$$
\operatorname{GIVIHFOWG}_{\lambda}\left(\widetilde{h}_{1}, \widetilde{h}_{2}, \ldots, \widetilde{h}_{n}\right)=\frac{1}{\lambda}\left(\bigotimes_{i=1}^{n}\left(\lambda \widetilde{h}_{\sigma(i)}\right)^{\omega_{i}}\right)
$$

with $\lambda>0$.

In Definition 24, if $\omega=(1 / n, 1 / n, \ldots, 1 / n)^{T}$, then the IVIHFOWA operator degenerates to the IVIHFA operator and the IVIHFOWG operator becomes the IVIHFG operator. If $\lambda=1$, then the GIVIHFOWA operator reduces to the IVIHFOWA operator and the GIVIHFOWG operator reduces to the IVIHFOWG operator.

Using IVIHFE operations and mathematical induction on $n$, (71), (72), (73), and (74) can be transformed into the following forms:

$$
\begin{aligned}
& \operatorname{IVIHFOWA}\left(\widetilde{h}_{1}, \widetilde{h}_{2}, \ldots, \widetilde{h}_{n}\right) \\
& =\left\{\left(\left[1-\prod_{i=1}^{n}\left(1-\mu_{\alpha_{\sigma(i)}}^{-}\right)^{\omega_{i}}, 1-\prod_{i=1}^{n}\left(1-\mu_{\alpha_{\sigma(i)}}^{+}\right)^{\omega_{i}}\right],\right.\right. \\
& \left.\quad\left[\prod_{i=1}^{n}\left(v_{\alpha_{\sigma(i)}}^{-}\right)^{\omega_{i}}, \prod_{i=1}^{n}\left(v_{\alpha_{\sigma(i)}^{+}}^{+}\right)^{\omega_{i}}\right]\right) \mid \\
& \left.\alpha_{\sigma(1)} \in \widetilde{h}_{\sigma(1)}, \alpha_{\sigma(2)} \in \widetilde{h}_{\sigma(2)}, \ldots, \alpha_{\sigma(n)} \in \widetilde{h}_{\sigma(n)}\right\},
\end{aligned}
$$$$
\operatorname{GIVIHFOWA}_{\lambda}\left(\widetilde{h}_{1}, \widetilde{h}_{2}, \ldots, \widetilde{h}_{n}\right)
$$$$
\begin{array}{r}
=\left\{\left(\left[1-\prod_{i=1}^{n}\left(1-\left(\mu_{\alpha_{\sigma(i)}^{-}}\right)^{\lambda}\right)^{\omega_{i}}\right)^{1 / \lambda},\right.\right. \\
\left.\left(1-\prod_{i=1}^{n}\left(1-\left(\mu_{\alpha_{\sigma(i)}^{+}}^{+}\right)^{\lambda}\right)^{\omega_{i}}\right)^{1 / \lambda}\right],
\end{array}
$$ 


$$
\begin{gathered}
\quad\left[1-\left(1-\prod_{i=1}^{n}\left(1-\left(1-v_{\alpha_{\sigma(i)}^{-}}^{-}\right)^{\lambda}\right)^{\omega_{i}}\right)^{1 / \lambda},\right. \\
\left.\left.1-\left(1-\prod_{i=1}^{n}\left(1-\left(1-v_{\alpha_{\sigma(i)}^{+}}^{+}\right)^{\lambda}\right)^{\omega_{i}}\right)^{1 / \lambda}\right]\right) \mid \\
\left.\alpha_{\sigma(1)} \in \widetilde{h}_{\sigma(1)}, \alpha_{\sigma(2)} \in \widetilde{h}_{\sigma(2)}, \ldots, \alpha_{\sigma(n)} \in \widetilde{h}_{\sigma(n)}\right\},
\end{gathered}
$$

IVIHFOWG $\left(\widetilde{h}_{1}, \widetilde{h}_{2}, \ldots, \widetilde{h}_{n}\right)$

$$
\begin{aligned}
&=\{\left(\left[\prod_{i=1}^{n}\left(\mu_{\alpha_{\sigma(i)}^{-}}\right)^{\omega_{i}}, \prod_{i=1}^{n}\left(\mu_{\alpha_{\sigma(i)}}^{+}\right)^{\omega_{i}}\right],\right. \\
& {\left.\left[1-\prod_{i=1}^{n}\left(1-v_{\alpha_{\sigma(i)}}^{-}\right)^{\omega_{i}}, 1-\prod_{i=1}^{n}\left(1-v_{\alpha_{\sigma(i)}}^{+}\right)^{\omega_{i}}\right]\right) \mid } \\
&\left.\alpha_{\sigma(1)} \in \widetilde{h}_{\sigma(1)}, \alpha_{\sigma(2)} \in \widetilde{h}_{\sigma(2)}, \ldots, \alpha_{\sigma(n)} \in \widetilde{h}_{\sigma(n)}\right\},
\end{aligned}
$$

$\operatorname{GIVIHFOWG}_{\lambda}\left(\widetilde{h}_{1}, \widetilde{h}_{2}, \ldots, \widetilde{h}_{n}\right)$

$$
\begin{gathered}
=\left\{\left(\left[1-\left(1-\prod_{i=1}^{n}\left(1-\left(1-\mu_{\alpha_{\sigma(i)}}^{-}\right)^{\lambda}\right)^{\omega_{i}}\right)^{1 / \lambda},\right.\right.\right. \\
\left.1-\left(1-\prod_{i=1}^{n}\left(1-\left(1-\mu_{\alpha_{\sigma(i)}^{+}}^{+}\right)^{\lambda}\right)^{\omega_{i}}\right)^{1 / \lambda}\right], \\
{\left[\left(1-\prod_{i=1}^{n}\left(1-\left(v_{\alpha_{\sigma(i)}^{-}}\right)^{\lambda}\right)^{\omega_{i}}\right)^{1 / \lambda},\right.} \\
\left.\left.\left(1-\prod_{i=1}^{n}\left(1-\left(v_{\alpha_{\sigma(i)}^{+}}\right)^{\lambda}\right)^{\omega_{i}}\right)^{1 / \lambda}\right]\right) \mid \\
\left.\alpha_{\sigma(1)} \in \widetilde{h}_{\sigma(1)}, \alpha_{\sigma(2)} \in \widetilde{h}_{\sigma(2)}, \ldots, \alpha_{\sigma(n)} \in \widetilde{h}_{\sigma(n)}\right\} .
\end{gathered}
$$

Remark 25. The IVIHFWA, IVIHFWG, GIVIHFWA, and GIVIHFWG operators weight only the interval-valued intuitionistic hesitant fuzzy arguments. However, by
Definition 24, the IVIHFOWA, IVIHFOWG, GIVIHFOWA, and GIVIHFOWG operators weight the ordered positions of the interval-valued intuitionistic hesitant fuzzy arguments instead of weighting the interval-valued intuitionistic hesitant fuzzy arguments themselves. The prominent characteristic of the IVIHFOWA, IVIHFOWG, GIVIHFOWA, and GIVIHFOWG operators is the reordering step in which the input arguments are rearranged in descending order; in particular, an interval-valued intuitionistic hesitant fuzzy argument $\widetilde{h}_{i}$ is not associated with a particular weight $w_{i}$, but rather a weight $w_{i}$ is associated with a particular ordered position $i$ of the interval-valued intuitionistic hesitant fuzzy arguments.

Example 26. Assume that $\widetilde{h}_{1}=\{([0.5,0.6],[0.3,0.4])$, $([0.7,0.8],[0.1,0.2]),([0.1,0.4],[0.5,0.6])\}, \widetilde{h}_{2}=\{([0.3,0.5]$, $[0.3,0.5]),([0.7,0.9],[0.1,0.1])\}$, and $\widetilde{h}_{3}=\{([0.2,0.4]$, $[0.1,0.3]),([0.6,0.8],[0.1,0.2])\}$ are three IVIHFEs, and the aggregation-associated vector is $\omega=(0.2,0.5,0.3)^{T}$. From Definition 24 , we can calculate the score values of $\widetilde{h}_{1}, \widetilde{h}_{2}$, and $\widetilde{h}_{3}$ as follows:

$$
\begin{gathered}
s\left(\widetilde{h}_{1}\right)=\frac{(0.2+0.2)+(0.6+0.6)+(-0.2-0.4)}{6}=0.1667, \\
s\left(\widetilde{h}_{2}\right)=\frac{(0+0)+(0.6+0.8)}{4}=0.3500, \\
s\left(\widetilde{h}_{3}\right)=\frac{(0.1+0.1)+(0.5+0.6)}{4}=0.3250 .
\end{gathered}
$$

Since $s\left(\widetilde{h}_{2}\right)>s\left(\widetilde{h}_{3}\right)>s\left(\widetilde{h}_{1}\right)$, then

$$
\begin{aligned}
\widetilde{h}_{\sigma(1)}=\widetilde{h}_{2}=\{([0.3,0.5],[0.3,0.5]),([0.7,0.9],[0.1,0.1])\}, \\
\widetilde{h}_{\sigma(2)}=\widetilde{h}_{3}=\{([0.2,0.4],[0.1,0.3]),([0.6,0.8],[0.1,0.2])\}, \\
\widetilde{h}_{\sigma(3)}=\widetilde{h}_{1} \\
=\{([0.5,0.6],[0.3,0.4]),([0.7,0.8],[0.1,0.2]), \\
\\
\quad([0.1,0.4],[0.5,0.6])\},
\end{aligned}
$$

$$
\begin{aligned}
& \operatorname{GIVIHFOWA}_{1}\left(\widetilde{h}_{1}, \widetilde{h}_{2}, \widetilde{h}_{3}\right) \\
& =\operatorname{IVIHFOWA}\left(\widetilde{h}_{1}, \widetilde{h}_{2}, \widetilde{h}_{3}\right) \\
& =\left\{\begin{array}{l}
([0.3235,0.4877],[0.1732,0.3622]),([0.4196,0.5839],[0.1246,0.2942]),([0.1931,0.4215],[0.2019,0.4091]), \\
([0.5217,0.7042],[0.1732,0.2958]),([0.5896,0.7598],[0.1246,0.2402]),([0.4294,0.6660],[0.2019,0.3340]), \\
([0.4290,0.6287],[0.1390,0.2625]),([0.5101,0.6984],[0.1000,0.2132]),([0.3189,0.5807],[0.1621,0.2965]), \\
([0.5962,0.7856],[0.1390,0.2144]),([0.6536,0.8259],[0.1000,0.1741]),([0.5184,0.7579],[0.1333,0.2421])
\end{array}\right\}, \\
& =\left\{\begin{array}{l}
([0.4224,0.5240],[0.1548,0.3451]),([0.5921,0.6845],[0.1178,0.2716]),([0.2431,0.4335],[0.1614,0.3599])), \\
([0.5661,0.7386],[0.1548,0.2614]),([0.6296,0.7783],[0.1178,0.2217]),([0.5442,0.7322],[0.1614,0.2678]), \\
([0.6147,0.8087],[0.1284,0.2262]),([0.6359,0.7843],[0.1000,0.1965]),([0.5590,0.7414],[0.1333,0.2308]),
\end{array}\right\},
\end{aligned}
$$




$$
\begin{aligned}
& \text { GIVIHFOWG }\left(\widetilde{h}_{1}, \widetilde{h}_{2}, \widetilde{h}_{3}\right) \\
& =\operatorname{IVIHFOWG}\left(\widetilde{h}_{1}, \widetilde{h}_{2}, \widetilde{h}_{3}\right) \\
& =\left\{\begin{array}{l}
([0.2855,0.4724],[0.2063,0.3751]),([0.3158,0.5149],[0.1441,0.3188]),([0.1762,0.4183],[0.2825,0.4467]), \\
([0.4945,0.6680],[0.2063,0.3320]),([0.5471,0.7282],[0.1441,0.2718]),([0.3051,0.5915],[0.2825,0.4085]), \\
([0.3382,0.5313],[0.1654,0.2972]),([0.3742,0.5792],[0.1000,0.2338]),([0.2087,0.4704],[0.2455,0.3777]), \\
([0.5858,0.7513],[0.1654,0.2487]),([0.6481,0.8191],[0.1000,0.1809]),([0.3615,0.6653],[0.2455,0.3347])
\end{array}\right\}, \\
& \text { GIVIHFOWG }\left(\widetilde{h}_{1}, \widetilde{h}_{2}, \widetilde{h}_{3}\right) \\
& =\left\{\begin{array}{l}
([0.2543,0.4455],[0.2717,0.4163]),([0.2560,0.4479],[0.2384,0.4014]),([0.1658,0.4131],[0.4223,0.5190]), \\
([0.4275,0.5866],[0.2717,0.4134]),([0.4371,0.6022],[0.2384,0.3978]),([0.2100,0.4817],[0.4223,0.5183]), \\
([0.2675,0.4534],[0.2526,0.3466]),([0.2694,0.4560],[0.1000,0.2731]),([0.1712,0.4186],[0.4212,0.5068]), \\
([0.5596,0.6626],[0.2526,0.3374]),([0.6312,0.8062],[0.1000,0.1938]),([0.2185,0.4940],[0.4212,0.5060])
\end{array}\right\} .
\end{aligned}
$$

Similar to Theorem 17, we have the following result.

Theorem 27. Let $\widetilde{h}_{i}(i=1,2, \ldots, n)$ be a collection of IVIH$F E s, \lambda>0$, and let $\omega=\left(\omega_{1}, \omega_{2}, \ldots, \omega_{n}\right)^{T}$ be the aggregationassociated vector such that $\omega_{i} \in[0,1]$ and $\sum_{i=1}^{n} \omega_{i}=1$; then the GIVIHFOWA operator is monotonically increasing with respect to the parameter $\lambda$.

Similar to Theorem 18, we have the following result.

Theorem 28. Let $\widetilde{h}_{i}(i=1,2, \ldots, n)$ be a collection of IVIHFEs, $\lambda>0$, and let $\omega=\left(\omega_{1}, \omega_{2}, \ldots, \omega_{n}\right)^{T}$ be the aggregationassociated vector such that $\omega_{i} \in[0,1]$ and $\sum_{i=1}^{n} \omega_{i}=1$; then the GIVIHFOWG operator is monotonically decreasing with respect to the parameter $\lambda$.

Similar to Theorems 20, 22, and 23 and Corollary 21, we can obtain the following theorem.

Theorem 29. Let $\widetilde{h}_{i}(i=1,2, \ldots, n)$ be a collection of IVIHFEs, $\lambda>0$, and let $\omega=\left(\omega_{1}, \omega_{2}, \ldots, \omega_{n}\right)^{T}$ be the aggregationassociated vector such that $\omega_{i} \in[0,1]$ and $\sum_{i=1}^{n} \omega_{i}=1$; then

$$
\begin{aligned}
& \operatorname{IVIHFOWG}\left(\widetilde{h}_{1}, \widetilde{h}_{2}, \ldots, \widetilde{h}_{n}\right) \\
& \quad \leq \operatorname{IVIHFOWA}\left(\widetilde{h}_{1}, \widetilde{h}_{2}, \ldots, \widetilde{h}_{n}\right), \\
& \operatorname{IVIHFOWG}\left(\widetilde{h}_{1}, \widetilde{h}_{2}, \ldots, \widetilde{h}_{n}\right) \\
& \quad \leq \operatorname{GIVIHFOWA}\left(\widetilde{h}_{1}, \widetilde{h}_{2}, \ldots, \widetilde{h}_{n}\right), \\
& \text { GIVIHFOWG } \\
& \left.\quad \leq \widetilde{h}_{1}, \widetilde{h}_{2}, \ldots, \widetilde{h}_{n}\right) \\
& \text { GIVIHIHFOWA }\left(\widetilde{h}_{1}, \widetilde{h}_{2}, \ldots, \widetilde{h}_{n}\right), \\
& \quad \leq \operatorname{GIVIHFOWA}\left(\widetilde{h}_{1}, \widetilde{h}_{2}, \ldots, \widetilde{h}_{n}\right)
\end{aligned}
$$

We now look at some special cases of the IVIHFOWA, IVIHFOWG, GIVIHFOWA, and GIVIHFOWG operators obtained by using different choices of the input arguments and the weight vector.

(1) If $\widetilde{h}_{i}(i=1,2, \ldots, n)$ is a collection of HFEs, then the IVIHFOWA operator reduces to the hesitant fuzzy ordered weighted averaging (HFOWA) operator [9], the IVIHFOWG operator reduces to the hesitant fuzzy ordered weighted geometric (HFOWG) operator [9], the GIVIHFOWA operator reduces to the generalized hesitant fuzzy ordered weighted averaging (GHFOWA) operator [9], and the GIVIHFOWG operator reduces to the generalized hesitant fuzzy ordered weighted geometric (GHFOWG) operator [9].

(2) If $\widetilde{h}_{i}(i=1,2, \ldots, n)$ is a collection of IVHFEs, then the IVIHFOWA operator reduces to the interval-valued hesitant fuzzy ordered weighted averaging (IVHFOWA) operator [6], the IVIHFOWG operator reduces to the interval-valued hesitant fuzzy ordered weighted geometric (IVHFOWG) operator [6], the GIVIHFOWA operator reduces to the generalized interval-valued hesitant fuzzy ordered weighted averaging (GIVHFOWA) operator [6], and the GIVIHFOWG operator reduces to the generalized interval-valued hesitant fuzzy ordered weighted geometric (GIVHFOWG) operator [6].

(3) If $\widetilde{h}_{i}(i=1,2, \ldots, n)$ is a collection of IHFEs, then the IVIHFOWA operator reduces to the intuitionistic hesitant fuzzy ordered weighted averaging (IHFOWA) operator:

$$
\begin{aligned}
& \operatorname{IHFOWA}\left(\widetilde{h}_{1}, \widetilde{h}_{2}, \ldots, \widetilde{h}_{n}\right) \\
& =\bigoplus_{i=1}^{n}\left(\omega_{i} \widetilde{h}_{\sigma(i)}\right) \\
& =\left\{\left(1-\prod_{i=1}^{n}\left(1-\mu_{\alpha_{\sigma(i)}}\right)^{\omega_{i}}, \prod_{i=1}^{n}\left(v_{\alpha_{\sigma(i)}}\right)^{\omega_{i}}\right) \mid\right. \\
& \left.\alpha_{\sigma(1)} \in \widetilde{h}_{\sigma(1)}, \alpha_{\sigma(2)} \in \widetilde{h}_{\sigma(2)}, \ldots, \alpha_{\sigma(n)} \in \widetilde{h}_{\sigma(n)}\right\} .
\end{aligned}
$$


If $\widetilde{h}_{i}(i=1,2, \ldots, n)$ is a collection of IHFEs, then the IVIHFOWG operator reduces to the intuitionistic hesitant fuzzy ordered weighted geometric (IHFOWG) operator:

$$
\begin{aligned}
& \operatorname{IHFOWG}\left(\widetilde{h}_{1}, \widetilde{h}_{2}, \ldots, \widetilde{h}_{n}\right) \\
& =\bigotimes_{i=1}^{n}\left(\widetilde{h}_{\sigma(i)}^{\omega_{i}}\right) \\
& =\left\{\left(\prod_{i=1}^{n}\left(\mu_{\alpha_{\sigma(i)}}\right)^{\omega_{i}}, 1-\prod_{i=1}^{n}\left(1-v_{\alpha_{\sigma(i)}}\right)^{\omega_{i}}\right) \mid\right. \\
& \left.\alpha_{\sigma(1)} \in \widetilde{h}_{\sigma(1)}, \alpha_{\sigma(2)} \in \widetilde{h}_{\sigma(2)}, \ldots, \alpha_{\sigma(n)} \in \widetilde{h}_{\sigma(n)}\right\} .
\end{aligned}
$$

If $\widetilde{h}_{i}(i=1,2, \ldots, n)$ is a collection of IHFEs, then the GIVIHFOWA operator reduces to the generalized intuitionistic hesitant fuzzy ordered weighted averaging (GIHFOWA) operator:

$$
\begin{aligned}
& \operatorname{GiHFOWA}\left(\widetilde{h}_{1}, \widetilde{h}_{2}, \ldots, \widetilde{h}_{n}\right) \\
& =\left(\bigoplus_{i=1}^{n}\left(\omega_{i} \widetilde{h}_{\sigma(i)}^{\lambda}\right)\right)^{1 / \lambda} \\
& =\left\{\left(1-\left(1-\prod_{i=1}^{n}\left(1-\left(1-\mu_{\alpha_{\sigma(i)}}\right)^{\lambda}\right)^{\omega_{i}}\right)^{1 / \lambda},\right.\right. \\
& \left.\left(1-\prod_{i=1}^{n}\left(1-\left(\nu_{\alpha_{\sigma(i)}}\right)^{\lambda}\right)^{\omega_{i}}\right)^{1 / \lambda}\right) \mid \\
& \left.\alpha_{\sigma(1)} \in \widetilde{h}_{\sigma(1)}, \alpha_{\sigma(2)} \in \widetilde{h}_{\sigma(2)}, \ldots, \alpha_{\sigma(n)} \in \widetilde{h}_{\sigma(n)}\right\} .
\end{aligned}
$$

If $\widetilde{h}_{i}(i=1,2, \ldots, n)$ is a collection of IHFEs, then the GIVIHFOWG operator reduces to the generalized intuitionistic hesitant fuzzy ordered weighted geometric (GIHFOWG) operator:

$$
\begin{aligned}
& \operatorname{GIHFOWG}\left(\widetilde{h}_{1}, \widetilde{h}_{2}, \ldots, \widetilde{h}_{n}\right) \\
& =\frac{1}{\lambda}\left(\bigotimes_{i=1}^{n}\left(\lambda \widetilde{h}_{\sigma(i)}\right)^{\omega_{i}}\right) \\
& =\left\{\left(1-\left(1-\prod_{i=1}^{n}\left(1-\left(1-\mu_{\alpha_{\sigma(i)}}\right)^{\lambda}\right)^{\omega_{i}}\right)^{1 / \lambda},\right.\right. \\
& \left.\left(1-\prod_{i=1}^{n}\left(1-\left(\nu_{\alpha_{\sigma(i)}}\right)^{\lambda}\right)^{\omega_{i}}\right)^{1 / \lambda}\right) \mid \\
& \left.\alpha_{\sigma(1)} \in \tilde{h}_{\sigma(1)}, \alpha_{\sigma(2)} \in \tilde{h}_{\sigma(2)}, \ldots, \alpha_{\sigma(n)} \in \tilde{h}_{\sigma(n)}\right\} .
\end{aligned}
$$

By Definitions 11, 13, 15, and 24, it is worth noting that all the operators mentioned previously have some inherent limitations. Concretely, IVIHFWA, IVIHFWG, GIVIHFWA, and GIVIHFWG operators only weight the interval-valued intuitionistic hesitant fuzzy argument itself but ignore the importance of the ordered position of the arguments, whereas the IVIHFOWA, IVIHFOWG, GIVIHFOWA, and GIVIHFOWG operators only weight the ordered position of each given argument but ignore the importance of the argument. To overcome this drawback, we present some hybrid aggregation operators for interval-valued intuitionistic hesitant fuzzy arguments, which weight not only all the given arguments but also their ordered positions.

\subsection{The IVIHFHA, IVIHFHG, GIVIHFHA, and GIVIHFHG Operators}

Definition 30. For a collection of IVIHFEs $\widetilde{h}_{i}(i=1,2, \ldots, n)$, $w=\left(w_{1}, w_{2}, \ldots, w_{n}\right)^{T}$ is the weight vector of them with $w_{i} \in$ $[0,1]$ and $\sum_{i=1}^{n} w_{i}=1$, and $n$ is the balancing coefficient which plays a role of balance; then we define the following aggregation operators, which are all based on the mapping $\widetilde{H}^{n} \rightarrow \widetilde{H}$ with an aggregation-associated vector $\omega=\left(\omega_{1}, \omega_{2}, \ldots, \omega_{n}\right)^{T}$ such that $\omega_{i} \in[0,1]$ and $\sum_{i=1}^{n} \omega_{i}=1$.

(1) The interval-valued intuitionistic hesitant fuzzy hybrid averaging (IVIHFHA) operator is

$$
\operatorname{IVIHFHA}\left(\widetilde{h}_{1}, \widetilde{h}_{2}, \ldots, \widetilde{h}_{n}\right)=\bigoplus_{i=1}^{n}\left(\omega_{i} \dot{\widetilde{h}}_{\sigma(i)}\right) \text {, }
$$

where $\dot{\tilde{h}}_{\sigma(i)}$ is the largest $i$ th of $\dot{\widetilde{h}}_{k}=n w_{k} \widetilde{h}_{k}(k=$ $1,2, \ldots, n)$.

(2) The interval-valued intuitionistic hesitant fuzzy hybrid geometric (IVIHFHG) operator is

$$
\operatorname{IVIHFHG}\left(\widetilde{h}_{1}, \widetilde{h}_{2}, \ldots, \widetilde{h}_{n}\right)=\bigotimes_{i=1}^{n}\left(\left(\ddot{\widetilde{h}}_{\sigma(i)}\right)^{\omega_{i}}\right)
$$

where $\ddot{\widetilde{h}}_{\sigma(i)}$ is the largest $i$ th of $\ddot{\widetilde{h}}_{k}=\left(\widetilde{h}_{k}\right)^{n w_{k}}(k=$ $1,2, \ldots, n)$.

(3) The generalized interval-valued intuitionistic hesitant fuzzy hybrid averaging (GIVIHFHA) operator is

$$
\operatorname{GIVIHFHA}_{\lambda}\left(\widetilde{h}_{1}, \widetilde{h}_{2}, \ldots, \widetilde{h}_{n}\right)=\left(\bigoplus_{i=1}^{n}\left(\omega_{i} \dot{\widetilde{h}}_{\sigma(i)}^{\lambda}\right)\right)^{1 / \lambda},
$$

where $\lambda>0$ and $\dot{\tilde{h}}_{\sigma(i)}$ is the largest $i$ th of $\dot{\tilde{h}}_{k}=n w_{k} \widetilde{h}_{k}$ $(k=1,2, \ldots, n)$. 
(4) The generalized interval-valued intuitionistic hesitant fuzzy hybrid geometric (GIVIHFHG) operator is

$$
\operatorname{GIVIHFHG}_{\lambda}\left(\widetilde{h}_{1}, \tilde{h}_{2}, \ldots, \tilde{h}_{n}\right)=\frac{1}{\lambda}\left(\bigotimes_{i=1}^{n}\left(\lambda \tilde{\tilde{h}}_{\sigma(i)}\right)^{\omega_{i}}\right),
$$

where $\lambda>0$ and $\ddot{h}_{\sigma(i)}$ is the largest $i$ th of $\tilde{h}_{k}=\left(\widetilde{h}_{k}\right)^{n w_{k}}$ $(k=1,2, \ldots, n)$.

If $w=(1 / n, 1 / n, \ldots, 1 / n)^{T}$ especially, then the IVIHFHA operator reduces to the IVIHFOWA operator, the IVIHFHG operator reduces to the IVIHFOWG operator, the GIVIHFHA operator reduces to the GIVIHFOWA operator, and the GIVIHFHG operator reduces to the GIVIHFOWG operator; if $\omega=(1 / n, 1 / n, \ldots, 1 / n)^{T}$, then the IVIHFHA operator reduces to the IVIHFWA operator, the IVIHFHG operator reduces to the IVIHFWG operator, the GIVIHFHA operator reduces to the GIVIHFWA operator, and the GIVIHFHG operator reduces to the GIVIHFWG operator; if $\lambda=1$, then the GIVIHFHA operator reduces to the IVIHFHA operator and the GIVIHFHG operator reduces to the IVIHFHG operator.

Using IVIHFE operations and mathematical induction on $n,(84),(85),(86)$, and (87) can be transformed into the following forms:

$$
\begin{aligned}
& \operatorname{IVIHFHA}\left(\tilde{h}_{1}, \tilde{h}_{2}, \ldots, \tilde{h}_{n}\right) \\
& \qquad\left\{\left(\left[1-\prod_{i=1}^{n}\left(1-\mu_{\dot{\alpha}_{\sigma(i)}^{-}}^{-}\right)^{\omega_{i}}, 1-\prod_{i=1}^{n}\left(1-\mu_{\dot{\alpha}_{\sigma(i)}^{+}}^{+}\right)^{\omega_{i}}\right],\right.\right. \\
& \left.\left.\qquad \prod_{i=1}^{n}\left(\nu_{\dot{\alpha}_{\sigma(i)}^{-}}\right)^{\omega_{i}}, \prod_{i=1}^{n}\left(\nu_{\dot{\alpha}_{\sigma(i)}^{+}}^{+}\right)^{\omega_{i}}\right]\right) \mid \\
& \left.\dot{\alpha}_{\sigma(1)} \in \dot{\tilde{h}}_{\sigma(1)}, \dot{\alpha}_{\sigma(2)} \in \dot{\tilde{h}}_{\sigma(2)}, \ldots, \dot{\alpha}_{\sigma(n)} \in \dot{\tilde{h}}_{\sigma(n)}\right\},
\end{aligned}
$$

$$
\begin{aligned}
& \operatorname{IVIHFHG}\left(\widetilde{h}_{1}, \widetilde{h}_{2}, \ldots, \widetilde{h}_{n}\right) \\
& =\left\{\left(\left[\prod_{i=1}^{n}\left(\mu_{\ddot{\alpha_{\sigma(i)}}}^{-}\right)^{\omega_{i}}, \prod_{i=1}^{n}\left(\mu_{\ddot{\alpha_{\sigma(i)}}}^{+}\right)^{\omega_{i}}\right],\right.\right. \\
& \left.\left[1-\prod_{i=1}^{n}\left(1-v_{\ddot{\alpha}_{\sigma(i)}}^{-}\right)^{\omega_{i}}, 1-\prod_{i=1}^{n}\left(1-v_{\dot{\alpha}_{\sigma(i)}}^{+}\right)^{\omega_{i}}\right]\right) \mid \\
& \left.\ddot{\alpha}_{\sigma(1)} \in \ddot{\tilde{h}}_{\sigma(1)}, \ddot{\alpha}_{\sigma(2)} \in \ddot{\widetilde{h}}_{\sigma(2)}, \ldots, \ddot{\alpha}_{\sigma(n)} \in \tilde{\widetilde{h}}_{\sigma(n)}\right\} \text {, }
\end{aligned}
$$

$$
\begin{aligned}
& \operatorname{GIVIHFHA}_{\lambda}\left(\widetilde{h}_{1}, \widetilde{h}_{2}, \ldots, \widetilde{h}_{n}\right) \\
& =\left\{\left(\left[\left(1-\prod_{i=1}^{n}\left(1-\left(\mu_{\dot{\alpha}_{\sigma(i)}}^{-}\right)^{\lambda}\right)^{\omega_{i}}\right)^{1 / \lambda}\right.\right.\right. \text {, } \\
& \left.\left(1-\prod_{i=1}^{n}\left(1-\left(\mu_{\alpha_{\sigma(i)}^{+}}^{+}\right)^{\lambda}\right)^{\omega_{i}}\right)^{1 / \lambda}\right] \\
& {\left[1-\left(1-\prod_{i=1}^{n}\left(1-\left(1-\nu_{\dot{\alpha}_{\sigma(i)}}^{-}\right)^{\lambda}\right)^{\omega_{i}}\right)^{1 / \lambda},\right.} \\
& \left.\left.1-\left(1-\prod_{i=1}^{n}\left(1-\left(1-\nu_{\dot{\alpha}_{\sigma(i)}^{+}}^{+}\right)^{\lambda}\right)^{\omega_{i}}\right)^{1 / \lambda}\right]\right) \mid \\
& \left.\dot{\alpha}_{\sigma(1)} \in \dot{\widetilde{h}}_{\sigma(1)}, \dot{\alpha}_{\sigma(2)} \in \dot{\tilde{h}}_{\sigma(2)}, \ldots, \dot{\alpha}_{\sigma(n)} \in \dot{\tilde{h}}_{\sigma(n)}\right\}
\end{aligned}
$$

$$
\begin{aligned}
& \operatorname{GIVIHFHG}_{\lambda}\left(\tilde{h}_{1}, \tilde{h}_{2}, \ldots, \tilde{h}_{n}\right) \\
& =\left\{\left(\left[1-\left(1-\prod_{i=1}^{n}\left(1-\left(1-\mu_{\alpha_{\sigma(i)}^{-}}^{-}\right)^{\lambda}\right)^{\omega_{i}}\right)^{1 / \lambda},\right.\right.\right. \\
& \left.1-\left(1-\prod_{i=1}^{n}\left(1-\left(1-\mu_{\alpha_{\sigma(i)}^{+}}^{+}\right)^{\lambda}\right)^{\omega_{i}}\right)^{1 / \lambda}\right], \\
& {\left[\left(1-\prod_{i=1}^{n}\left(1-\left(\nu_{\ddot{\alpha}_{\sigma(i)}^{-}}^{-}\right)^{\lambda}\right)^{\omega_{i}}\right)^{1 / \lambda},\right.} \\
& \left.\left.\left(1-\prod_{i=1}^{n}\left(1-\left(v_{\dot{\alpha}_{\sigma(i)}^{+}}^{+}\right)^{\lambda}\right)^{\omega_{i}}\right)^{1 / \lambda}\right]\right) \mid \\
& \left.\ddot{\alpha}_{\sigma(1)} \in \ddot{\widetilde{h}}_{\sigma(1)}, \ddot{\alpha}_{\sigma(2)} \in \ddot{\tilde{h}}_{\sigma(2)}, \ldots, \ddot{\alpha}_{\sigma(n)} \in \ddot{\tilde{h}}_{\sigma(n)}\right\} .
\end{aligned}
$$

Example 31. Let $\tilde{h}_{1}=\{([0.1,0.2],[0.5,0.7]),([0.6,0.9]$, $[0.1,0.1])\}, \widetilde{h}_{2}=\{([0.5,0.6],[0.4,0.4]),([0.7,0.8],[0.1,0.2])\}$, and $\tilde{h}_{3}=\{([0.6,0.7],[0.2,0.3]),([0.2,0.5],[0.3,0.4])$, $([0.4,0.6],[0.2,0.3])\}$ be three IVIHFEs, whose weight vector is $w=(0.1,0.5,0.4)^{T}$, and the aggregation-associated vector is $\omega=(0.3,0.5,0.2)^{T}$. Then we can obtain

$$
\begin{aligned}
\tilde{h}_{1}= & 3 \cdot 0.1 \cdot \tilde{h}_{1} \\
= & \left\{\left(\left[1-\left(1-\mu_{\alpha}^{-}\right)^{0.3}, 1-\left(1-\mu_{\alpha}^{+}\right)^{0.3}\right],\right.\right. \\
& \left.\left.\quad\left[\left(\nu_{\alpha}^{-}\right)^{0.3},\left(\nu_{\alpha}^{+}\right)^{0.3}\right]\right) \mid \alpha \in \tilde{h}_{1}\right\} \\
= & \{([0.0311,0.0648],[0.8123,0.8985]), \\
& ([0.2403,0.4988],[0.5012,0.5012])\},
\end{aligned}
$$




$$
\begin{aligned}
\dot{\tilde{h}}_{2}= & 3 \cdot 0.5 \cdot \tilde{h}_{2} \\
= & \left\{\left(\left[1-\left(1-\mu_{\alpha}^{-}\right)^{1.5}, 1-\left(1-\mu_{\alpha}^{+}\right)^{1.5}\right],\right.\right. \\
& {\left.\left.\left[\left(v_{\alpha}^{-}\right)^{1.5},\left(v_{\alpha}^{+}\right)^{1.5}\right]\right) \mid \alpha \in \widetilde{h}_{2}\right\} } \\
= & \{([0.6464,0.7470],[0.2530,0.2530]), \\
& ([0.8357,0.9106],[0.0316,0.0894])\}, \\
\dot{\widetilde{h}}_{3}=3 \cdot 0.4 \cdot \widetilde{h}_{3} & \left\{\left(\left[1-\left(1-\mu_{\alpha}^{-}\right)^{1.2}, 1-\left(1-\mu_{\alpha}^{+}\right)^{1.2}\right],\right.\right. \\
= & {\left.\left.\left[\left(v_{\alpha}^{-}\right)^{1.2},\left(v_{\alpha}^{+}\right)^{1.2}\right]\right) \mid \alpha \in \widetilde{h}_{3}\right\} } \\
= & \{([0.6670,0.7642],[0.1450,0.2358]), \\
& ([0.2349,0.5647],[0.2358,0.3330]), \\
& ([0.4583,0.6670],[0.1450,0.2358])\}, \\
s\left(\dot{\tilde{h}}_{1}\right)=-0.4695, \quad s\left(\tilde{h}_{2}\right)=0.6282, & s\left(\tilde{h}_{3}\right)=0.3376 .
\end{aligned}
$$

$$
\text { Since } s\left(\tilde{h}_{2}\right)>s\left(\dot{\widetilde{h}}_{3}\right)>s\left(\dot{\widetilde{h}}_{1}\right) \text {, then we have }
$$$$
\tilde{h}_{\sigma(1)}=\tilde{h}_{2}
$$$$
=\{([0.6464,0.7470],[0.2530,0.2530]),
$$$$
([0.8357,0.9106],[0.0316,0.0894])\} \text {, }
$$$$
\dot{\tilde{h}}_{\sigma(2)}=\dot{\tilde{h}}_{3}
$$$$
=\{([0.6670,0.7642],[0.1450,0.2358]),
$$$$
\text { ([0.2349, 0.5647] , [0.2358, 0.3330]), }
$$$$
([0.4583,0.6670],[0.1450,0.2358])\} \text {, }
$$$$
\dot{\tilde{h}}_{\sigma(3)}=\dot{\tilde{h}}_{1}
$$$$
=\{([0.0311,0.0648],[0.8123,0.8985]),
$$$$
([0.2403,0.4988],[0.5012,0.5012])\} \text {. }
$$

According to Definition 30, we can obtain

$$
\begin{aligned}
& \operatorname{GIVIHFHA}_{1}\left(\widetilde{h}_{1}, \widetilde{h}_{2}, \widetilde{h}_{3}\right) \\
& =\operatorname{IVIHFHA}\left(\widetilde{h}_{1}, \widetilde{h}_{2}, \widetilde{h}_{3}\right) \\
& =\left\{\begin{array}{l}
([0.3579,0.4634],[0.3937,0.4931]),([0.5387,0.6969],[0.2628,0.3031]),([0.2124,0.3983],[0.4268,0.5265]), \\
([0.3197,0.5991],[0.3031,0.3489]),([0.2967,0.4329],[0.3937,0.4931]),([0.4466,0.6511],[0.2628,0.3031]), \\
([0.3866,0.4917],[0.3446,0.4621]),([0.5819,0.7396],[0.2031,0.2604]),([0.2294,0.4227],[0.3804,0.4975]), \\
([0.3453,0.6358],[0.2466,0.3091]),([0.3204,0.4594],[0.3446,0.4621]),([0.4823,0.6909],[0.2031,0.2604])
\end{array}\right\}, \\
& \text { GIVIHFHA } \left.6 \text { ( } \widetilde{h}_{1}, \widetilde{h}_{2}, \widetilde{h}_{3}\right) \\
& =\left\{\begin{array}{l}
([0.1831,0.2362],[0.6350,0.7196]),([0.4081,0.6131],[0.3853,0.3869]),([0.1523,0.2345],[0.6351,0.7201]), \\
([0.2762,0.5662],[0.3869,0.3976]),([0.1793,0.2359],[0.6350,0.7196]),([0.3842,0.6024],[0.3853,0.3869]), \\
([0.1833,0.2363],[0.6349,0.7196]),([0.4094,0.6146],[0.3838,0.3854]),([0.1524,0.2346],[0.6350,0.7200]),
\end{array}\right\} .
\end{aligned}
$$

If we utilize the GIVIHFHG operator to aggregate the three IVIHFEs $\widetilde{h}_{1}, \widetilde{h}_{2}$, and $\widetilde{h}_{3}$, then we have

$$
\begin{aligned}
\ddot{h}_{1}= & \left(\widetilde{h}_{1}\right)^{3 \cdot 0.1} \\
= & \left\{\left(\left[\left(\mu_{\alpha}^{-}\right)^{0.3},\left(\mu_{\alpha}^{+}\right)^{0.3}\right],\right.\right. \\
& \left.\quad\left[1-\left(1-\nu_{\alpha}^{-}\right)^{0.3}, 1-\left(1-v_{\alpha}^{+}\right)^{0.3}\right]\right) \mid \\
& \left.\alpha \in \widetilde{h}_{1}\right\} \\
= & \{([0.5012,0.6170],[0.1877,0.3032]), \\
& ([0.8579,0.9689],[0.0311,0.0311])\},
\end{aligned}
$$

$$
\begin{aligned}
\ddot{h}_{2}= & \left(\widetilde{h}_{2}\right)^{3 \cdot 0.5} \\
= & \left\{\left(\left[\left(\mu_{\alpha}^{-}\right)^{1.5},\left(\mu_{\alpha}^{+}\right)^{1.5}\right],\right.\right. \\
& {\left.\left[1-\left(1-v_{\alpha}^{-}\right)^{1.5}, 1-\left(1-v_{\alpha}^{+}\right)^{1.5}\right]\right) \mid } \\
& \left.\alpha \in \widetilde{h}_{2}\right\} \\
= & \{([0.3536,0.4648],[0.5352,0.5352]), \\
& ([0.5857,0.7155],[0.1462,0.2845])\},
\end{aligned}
$$




$$
\begin{gathered}
\ddot{\widetilde{h}}_{3}=\left(\widetilde{h}_{3}\right)^{3 \cdot 0.4} \\
=\left\{\left(\left[\left(\mu_{\alpha}^{-}\right)^{1.2},\left(\mu_{\alpha}^{+}\right)^{1.2}\right],\right.\right. \\
\left.\quad\left[1-\left(1-v_{\alpha}^{-}\right)^{1.2}, 1-\left(1-v_{\alpha}^{+}\right)^{1.2}\right]\right) \mid \\
\left.\alpha \in \widetilde{h}_{3}\right\} \\
=\{([0.5417,0.6518],[0.2349,0.3482]), \\
([0.1450,0.4353],[0.3482,0.4583]), \\
([0.3330,0.5417],[0.2349,0.3482])\}, \\
s\left(\ddot{\tilde{h}_{1}}\right)=0.5980, \quad s(\ddot{\widetilde{h}})=0.1546, \\
s\left(\ddot{\tilde{h}_{3}}\right)=0.1126 .
\end{gathered}
$$

$$
\begin{aligned}
\ddot{h}_{\sigma(2)}= & \ddot{h}_{2} \\
= & \{([0.3536,0.4648],[0.5352,0.5352]), \\
& ([0.5857,0.7155],[0.1462,0.2845])\}, \\
\ddot{\tilde{h}}_{\sigma(3)}= & \widetilde{\widetilde{h}}_{3} \\
=\{ & \{([0.5417,0.6518],[0.2349,0.3482]), \\
& ([0.1450,0.4353],[0.3482,0.4583]), \\
& ([0.3330,0.5417],[0.2349,0.3482])\} .
\end{aligned}
$$

According to Definition 30, we can obtain

$$
\begin{aligned}
& \text { GIVIHFHG }_{1}\left(\widetilde{h}_{1}, \widetilde{h}_{2}, \widetilde{h}_{3}\right) \\
& =\operatorname{IVIHFHG}\left(\widetilde{h}_{1}, \widetilde{h}_{2}, \widetilde{h}_{3}\right) \\
& =\left\{\begin{array}{l}
([0.4417,0.5558],[0.3315,0.4141]),([0.3676,0.5107],[0.3586,0.4375]),([0.3982,0.5307],[0.3315,0.4141]), \\
([0.5530,0.6761],[0.1733,0.3019]),([0.4937,0.6432],[0.1874,0.3190]),([0.5182,0.6578],[0.1733,0.3019]), \\
([0.6169,0.7909],[0.1933,0.2091]),([0.5661,0.7696],[0.2091,0.2210]),([0.5871,0.7790],[0.1933,0.2091]), \\
([0.6933,0.8475],[0.1010,0.1525]),([0.6526,0.8320],[0.1093,0.1611]),([0.6694,0.8389],[0.1010,0.1525])
\end{array}\right\}, \\
& =\left\{\begin{array}{l}
([0.4704,0.5781],[0.2790,0.3817]),([0.4233,0.5340],[0.3022,0.4023]),([0.4266,0.5472],[0.2790,0.3817]), \\
([0.5576,0.6809],[0.1713,0.3006]),([0.5410,0.6650],[0.1800,0.3106]),([0.5420,0.6693],[0.1713,0.3006]), \\
([0.7393,0.8660],[0.1285,0.1340]),([0.7226,0.8631],[0.1340,0.1362]),([0.7228,0.8638],[0.1285,0.1340]),
\end{array}\right\} .
\end{aligned}
$$

We now look at some special cases of the IVIHFHA, IVIHFHG, GIVIHFHA, and GIVIHFHG operators obtained by using different choices of the input arguments and the weight vector.

(1) If $\widetilde{h}_{i}(i=1,2, \ldots, n)$ is a collection of HFEs, then the IVIHFHA operator reduces to the hesitant fuzzy hybrid averaging (HFHA) operator [9], the IVIHFHG operator reduces to the hesitant fuzzy hybrid geometric (HFHG) operator [9], the GIVIHFHA operator reduces to the generalized hesitant fuzzy hybrid averaging (GHFHA) operator [9], and the GIVIHFHG operator reduces to the generalized hesitant fuzzy hybrid geometric (GHFHG) operator [9].

(2) If $\widetilde{h}_{i}(i=1,2, \ldots, n)$ is a collection of IVHFEs, then the IVIHFHA operator reduces to the interval-valued hesitant fuzzy hybrid averaging (IVHFHA) operator [6], the IVIHFHG operator reduces to the interval-valued hesitant fuzzy hybrid geometric (IVHFHG) operator [6], the GIVIHFHA operator reduces to the generalized interval-valued hesitant fuzzy hybrid averaging (GIVHFHA) operator [6], and the GIVIHFHG operator reduces to the generalized interval-valued hesitant fuzzy hybrid geometric (GIVHFHG) operator [6].

(3) If $\widetilde{h}_{i}(i=1,2, \ldots, n)$ is a collection of IHFEs, then the IVIHFHA operator reduces to the intuitionistic hesitant fuzzy hybrid averaging (IHFHA) operator:

$$
\begin{aligned}
& \operatorname{IHFHA}\left(\widetilde{h}_{1}, \widetilde{h}_{2}, \ldots, \widetilde{h}_{n}\right) \\
& =\bigoplus_{i=1}^{n}\left(\omega_{i} \dot{\widetilde{h}}_{\sigma(i)}\right) \\
& =\left\{\left(1-\prod_{i=1}^{n}\left(1-\mu_{\dot{\alpha}_{\sigma(i)}}\right)^{\omega_{i}}, \prod_{i=1}^{n}\left(v_{\dot{\alpha}_{\sigma(i)}}\right)^{\omega_{i}}\right) \mid\right. \\
& \left.\dot{\alpha}_{\sigma(1)} \in \dot{\widetilde{h}}_{\sigma(1)}, \dot{\alpha}_{\sigma(2)} \in \dot{\widetilde{h}}_{\sigma(2)}, \ldots, \dot{\alpha}_{\sigma(n)} \in \dot{\widetilde{h}}_{\sigma(n)}\right\} .
\end{aligned}
$$


If $\widetilde{h}_{i}(i=1,2, \ldots, n)$ is a collection of IHFEs, then the IVIHFHG operator reduces to the intuitionistic hesitant fuzzy hybrid geometric (IHFHG) operator:

$$
\begin{aligned}
& \operatorname{IHFHG}\left(\tilde{h}_{1}, \tilde{h}_{2}, \ldots, \tilde{h}_{n}\right) \\
&= \bigotimes_{i=1}^{n}\left(\left(\tilde{h}_{\sigma(i)}\right)^{\omega_{i}}\right) \\
&=\left\{\left(\prod_{i=1}^{n}\left(\mu_{\ddot{\alpha}_{\sigma(i)}}\right)^{\omega_{i}}, 1-\prod_{i=1}^{n}\left(1-\nu_{\ddot{\alpha}_{\sigma(i)}}\right)^{\omega_{i}}\right) \mid\right. \\
&\left.\ddot{\alpha}_{\sigma(1)} \in \tilde{\widetilde{h}}_{\sigma(1)}, \ddot{\alpha}_{\sigma(2)} \in \tilde{\widetilde{h}}_{\sigma(2)}, \ldots, \ddot{\alpha}_{\sigma(n)} \in \tilde{\widetilde{h}}_{\sigma(n)}\right\} .
\end{aligned}
$$

If $\tilde{h}_{i}(i=1,2, \ldots, n)$ is a collection of IHFEs, then the GIVIHFHA operator reduces to the generalized intuitionistic hesitant fuzzy hybrid averaging (GIHFHA) operator:

$$
\begin{aligned}
& \operatorname{GIHFHA}_{\lambda}\left(\widetilde{h}_{1}, \tilde{h}_{2}, \ldots, \tilde{h}_{n}\right) \\
& =\left(\bigoplus_{i=1}^{n}\left(\omega_{i} \tilde{\tilde{h}}_{\sigma(i)}^{\lambda}\right)\right)^{1 / \lambda} \\
& =\left\{\left(\left(1-\prod_{i=1}^{n}\left(1-\left(\mu_{\dot{\alpha}_{\sigma(i)}}\right)^{\lambda}\right)^{\omega_{i}}\right)^{1 / \lambda},\right.\right. \\
& \left.\dot{\alpha}\left(1-\prod_{i=1}^{n}\left(1-\left(1-\nu_{\dot{\alpha}_{\sigma(i)}}\right)^{\lambda}\right)^{\omega_{i}}\right)^{1 / \lambda}\right) \mid \\
& \left.\dot{\tilde{h}}_{\sigma(1)}, \dot{\alpha}_{\sigma(2)} \in \dot{\tilde{h}}_{\sigma(2)}, \ldots, \dot{\alpha}_{\sigma(n)} \in \dot{\tilde{h}}_{\sigma(n)}\right\} .
\end{aligned}
$$

If $\tilde{h}_{i}(i=1,2, \ldots, n)$ is a collection of IHFEs, then the GIVIHFHG operator reduces to the generalized intuitionistic hesitant fuzzy hybrid geometric (GIHFHG) operator:

$$
\begin{aligned}
\operatorname{GIHFHG}_{\lambda}\left(\tilde{h}_{1}, \tilde{h}_{2}, \ldots, \tilde{h}_{n}\right) \\
=\frac{1}{\lambda}\left(\bigotimes_{i=1}^{n}\left(\lambda \tilde{\bar{h}}_{\sigma(i)}\right)^{\omega_{i}}\right) \\
=\left\{\left(1-\left(1-\prod_{i=1}^{n}\left(1-\left(1-\mu_{\ddot{\alpha}_{\sigma(i)}}\right)^{\lambda}\right)^{\omega_{i}}\right)^{1 / \lambda},\right.\right. \\
\left.\left(1-\prod_{i=1}^{n}\left(1-\left(\nu_{\ddot{\alpha}_{\sigma(i)}}\right)^{\lambda}\right)^{\omega_{i}}\right)^{1 / \lambda}\right) \mid \\
\left.\ddot{\alpha}_{\sigma(1)} \in \tilde{\tilde{h}}_{\sigma(1)}, \ddot{\alpha}_{\sigma(2)} \in \tilde{\tilde{h}}_{\sigma(2)}, \ldots, \ddot{\alpha}_{\sigma(n)} \in \tilde{\tilde{h}}_{\sigma(n)}\right\} .
\end{aligned}
$$

In the present section, we introduce twelve kinds of aggregation operators for aggregating the interval-valued
TABLE 1: Interval-valued intuitionistic hesitant fuzzy aggregation operators.

\begin{tabular}{lc}
\hline Order & The names of operators \\
\hline 1 & The IVIHFWA operator \\
2 & The IVIHFWG operator \\
3 & The GIVIHFWA operator \\
4 & The GIVIHFWG operator \\
5 & The IVIHFOWA operator \\
6 & The IVIHFOWG operator \\
7 & The GIVIHFOWA operator \\
8 & The GIVIHFOWG operator \\
9 & The IVIHFHA operator \\
10 & The IVIHFHG operator \\
11 & The GIVIHFHA operator \\
12 & The GIVIHFHG operator \\
\hline
\end{tabular}

intuitionistic hesitant fuzzy information. To exhibit these operators more clearly, we list all of them in Table 1.

\section{An Approach to Multiple Attribute Group Decision-Making with Interval-Valued Intuitionistic Hesitant Fuzzy Information}

In this section, we utilize the proposed interval-valued intuitionistic hesitant fuzzy aggregation operators to develop an approach to multiple attribute group decision-making with interval-valued intuitionistic hesitant fuzzy information. First, a multiple attribute group decision-making with interval-valued intuitionistic hesitant fuzzy information can be described as follows.

Let $Y=\left\{Y_{1}, Y_{2}, \ldots, Y_{m}\right\}$ be a set of $m$ alternatives, $G=$ $\left\{G_{1}, G_{2}, \ldots, G_{n}\right\}$ a collection of $n$ attributes, whose weight vector is $w=\left(w_{1}, w_{2}, \ldots, w_{n}\right)^{T}$, with $w_{i} \in[0,1], i=1,2$, $\ldots, n$, and $\sum_{i=1}^{n} w_{i}=1$, and let $D=\left\{D_{1}, D_{2}, \ldots, D_{l}\right\}$ be a set of $l$ decision makers, whose weight vector is $\omega=$ $\left(\omega_{1}, \omega_{2}, \ldots, \omega_{l}\right)^{T}$, with $\omega_{k} \in[0,1], k=1,2, \ldots, l$, and $\sum_{k=1}^{l} \omega_{k}=1$. Let $\widetilde{R}^{(k)}=\left(\widetilde{r}_{i j}^{(k)}\right)_{m \times n}$ be an interval-valued intuitionistic hesitant fuzzy decision matrix, where $\widetilde{r}_{i j}^{(k)}=\left\{\gamma_{i j}^{(k)} \mid\right.$ $\left.\gamma_{i j}^{(k)} \in \widetilde{r}_{i j}^{(k)}\right\}=\left\{\left(\left[\mu_{\gamma_{i j}^{(k)}}^{-}, \mu_{\gamma_{i j}^{(k)}}^{+}\right],\left[\nu_{\gamma_{i j}^{(k)}}^{-}, \nu_{\gamma_{i j}^{(k)}}^{+}\right]\right) \mid \gamma_{i j}^{(k)} \in \widetilde{r}_{i j}^{(k)}\right\} \in \widetilde{H}$ is an IVIHFE given by the decision maker $D_{k} \in D$, where $\left[\mu_{\gamma_{i j}^{(k)}}^{-}, \mu_{\gamma_{i j}^{(k)}}^{+}\right]$indicates the possible degree range that the alternative $Y_{i} \in Y$ satisfies the attribute $G_{j} \in G$, while $\left[v_{\gamma_{i j}^{(k)}}^{-}, \nu_{\gamma_{i j}^{(k)}}^{+}\right]$ indicates the possible degree range that the alternative $Y_{i} \in Y$ does not satisfy the attribute $G_{j} \in G$.

In general, there are benefit attributes (i.e., the bigger the attribute values, the better) and cost attributes (i.e., the smaller the attribute values, the better) in a multiple attribute group decision-making problem. In such cases, we transform the attribute values of cost type into the attribute values of benefit type; that is, normalize the interval-valued intuitionistic hesitant fuzzy decision matrix $\widetilde{R}^{(k)}=\left(\widetilde{r}_{i j}^{(k)}\right)_{m \times n}$ into a corresponding interval-valued intuitionistic hesitant fuzzy 
decision matrix $\widetilde{A}^{(k)}=\left(\widetilde{a}_{i j}^{(k)}\right)_{m \times n}=\left(\left\{\alpha_{i j}^{(k)} \mid \alpha_{i j}^{(k)} \in \widetilde{a}_{i j}^{(k)}\right\}\right)_{m \times n}=$ $\left(\left\{\left(\left[\mu_{\alpha_{i j}^{(k)}}^{-}, \mu_{\alpha_{i j}^{(k)}}^{+}\right],\left[\nu_{\alpha_{i j}^{(k)}}^{-}, v_{\alpha_{i j}^{(k)}}^{+}\right]\right) \mid \alpha_{i j}^{(k)} \in \widetilde{a}_{i j}^{(k)}\right\}\right)$ mn by the method given in $[16,17]$, where

$$
\begin{gathered}
\widetilde{a}_{i j}^{(k)}= \begin{cases}\widetilde{r}_{i j}^{(k)}, & \text { for benefit attribute } G_{j}, \\
\left(\widetilde{r}_{i j}^{(k)}\right)^{c}, & \text { for cost attribute } G_{j},\end{cases} \\
i=1,2, \ldots, m, j=1,2, \ldots, n, k=1,2, \ldots, l,
\end{gathered}
$$

where $\left(\widetilde{r}_{i j}^{(k)}\right)^{c}$ is the complement of $\widetilde{r}_{i j}^{(k)}$ such that

$$
\begin{aligned}
\left(\widetilde{r}_{i j}^{(k)}\right)^{c} & =\left\{\left(\gamma_{i j}^{(k)}\right)^{c} \mid \gamma_{i j}^{(k)} \in \widetilde{r}_{i j}^{(k)}\right\} \\
& =\left\{\left(\left[v_{\gamma_{i j}^{(k)}}^{-}, v_{\gamma_{i j}}^{+}\right],\left[\mu_{\gamma_{i j}^{(k)}}^{-}, \mu_{\gamma_{i j}^{(k)}}^{+}\right]\right) \mid \gamma_{i j}^{(k)} \in \widetilde{r}_{i j}^{(k)}\right\} .
\end{aligned}
$$

In the following, we utilize the proposed operators to develop an approach to multiple attribute group decisionmaking with interval-valued intuitionistic hesitant fuzzy information, which involves the following steps.

Algorithm 32. Consider the following.

Step 1. Transform the decision matrix $\widetilde{R}^{(k)}=\left(\widetilde{r}_{i j}^{(k)}\right)_{m \times n}$ into a normalized matrix $\widetilde{A}^{(k)}=\left(\widetilde{a}_{i j}^{(k)}\right)_{m \times n}=\left(\left\{\alpha_{i j}^{(k)} \mid \alpha_{i j}^{(k)} \epsilon\right.\right.$ $\left.\left.\tilde{a}_{i j}^{(k)}\right\}\right)_{m \times n}=\left(\left\{\left(\left[\mu_{\alpha_{i j}^{(k)}}^{-}, \mu_{\alpha_{i j}^{(k)}}^{+}\right],\left[\nu_{\alpha_{i j}^{(k)}}^{-}, \nu_{\alpha_{i j}^{(k)}}^{+}\right]\right) \mid \alpha_{i j}^{(k)} \in \tilde{a}_{i j}^{(k)}\right\}\right)_{m \times n}$ based on (102).

Step 2. Utilize the GIVIHFWA operator (26):

$$
\begin{gathered}
\tilde{a}_{i j}=\operatorname{GIVIHFWA}_{\lambda}\left(\widetilde{a}_{i j}^{(1)}, \widetilde{a}_{i j}^{(2)}, \ldots, \widetilde{a}_{i j}^{(l)}\right) \\
=\left\{\left(\left[1-\prod_{k=1}^{l}\left(1-\left(\mu_{\alpha_{i j}^{(k)}}^{-}\right)^{\lambda}\right)^{\omega_{k}}\right)^{1 / \lambda},\right.\right. \\
\left.\left(1-\prod_{k=1}^{l}\left(1-\left(\mu_{\alpha_{i j}^{+}}^{+}\right)^{\lambda}\right)^{\omega_{k}}\right)^{1 / \lambda}\right], \\
{\left[1-\left(1-\prod_{k=1}^{l}\left(1-\left(1-v_{\alpha_{i j}^{(k)}}^{-}\right)^{\lambda}\right)^{\omega_{k}}\right)^{1 / \lambda},\right.} \\
\left.\left.\quad 1-\left(1-\prod_{k=1}^{l}\left(1-\left(1-v_{\alpha_{i j}^{+}}^{+}\right)^{\lambda}\right)^{\lambda}\right)^{\omega_{k}}\right]\right) \mid \\
\left.\alpha_{i j}^{(1)} \in \widetilde{a}_{i j}^{(1)}, \alpha_{i j}^{(2)} \in \widetilde{a}_{i j}^{(2)}, \ldots, \alpha_{i j}^{(l)} \in \widetilde{a}_{i j}^{(l)}\right\}, \\
i=1,2, \ldots, m, \quad j=1,2, \ldots, n,
\end{gathered}
$$

or the GIVIHFWG operator (27):

$$
\begin{gathered}
\widetilde{a}_{i j}=\operatorname{GIVIHFWG}_{\lambda}\left(\widetilde{a}_{i j}^{(1)}, \widetilde{a}_{i j}^{(2)}, \ldots, \widetilde{a}_{i j}^{(l)}\right) \\
=\left\{\left(\left[1-\left(1-\prod_{k=1}^{l}\left(1-\left(1-\mu_{\alpha_{i j}^{(k)}}^{-}\right)^{\lambda}\right)^{\omega_{k}}\right)^{1 / \lambda},\right.\right.\right. \\
\left.1-\left(1-\prod_{k=1}^{l}\left(1-\left(1-\mu_{\alpha_{i j}^{+(k)}}^{+}\right)^{\lambda}\right)^{\omega_{k}}\right)^{1 / \lambda}\right], \\
{\left[\left(1-\prod_{k=1}^{l}\left(1-\left(v_{\alpha_{i j}^{(k)}}^{-}\right)^{\lambda}\right)^{\omega_{k}}\right)^{1 / \lambda},\right.} \\
\left.\left.\quad\left(1-\prod_{k=1}^{l}\left(1-\left(v_{\alpha_{i j}^{(k)}}^{+}\right)^{\lambda}\right)^{\omega_{k}}\right)^{1 / \lambda}\right]\right) \mid \\
\left.\alpha_{i j}^{(1)} \in \widetilde{a}_{i j}^{(1)}, \alpha_{i j}^{(2)} \in \widetilde{a}_{i j}^{(2)}, \ldots, \alpha_{i j}^{(l)} \in \widetilde{a}_{i j}^{(l)}\right\}, \\
i=1,2, \ldots, m, \quad j=1,2, \ldots, n,
\end{gathered}
$$

to aggregate all the individual interval-valued intuitionistic hesitant fuzzy decision matrix $\widetilde{A}^{(k)}=\left(\widetilde{a}_{i j}^{(k)}\right)_{m \times n}(k=1,2, \ldots, l)$ into the collective interval-valued intuitionistic hesitant fuzzy decision matrix $\widetilde{A}=\left(\widetilde{a}_{i j}\right)_{m \times n}=\left(\left\{\alpha_{i j} \mid \alpha_{i j} \in \widetilde{a}_{i j}\right\}\right)_{m \times n}=$ $\left(\left\{\left(\left[\mu_{\alpha_{i j}}^{-}, \mu_{\alpha_{i j}}^{+}\right],\left[v_{\alpha_{i j}}^{-}, v_{\alpha_{i j}}^{+}\right]\right) \mid \alpha_{i j} \in \widetilde{a}_{i j}\right\}\right)_{m \times n}$.

Step 3. Utilize the GIVIHFHA operator (90):

$$
\begin{gathered}
\widetilde{a}_{i}=\operatorname{GIVIHFHA}_{\lambda}\left(\widetilde{a}_{i 1}, \tilde{a}_{i 2}, \ldots, \widetilde{a}_{i n}\right) \\
=\left\{\left(1-\prod_{j=1}^{n}\left(1-\left(\mu_{\dot{\alpha}_{i \sigma(j)}^{-}}\right)^{\lambda}\right)^{\xi_{j}}\right)^{1 / \lambda},\right. \\
\left.\left(1-\prod_{j=1}^{n}\left(1-\left(\mu_{\dot{\alpha}_{i \sigma(j)}^{+}}\right)^{\lambda}\right)^{\xi_{j}}\right)^{1 / \lambda}\right], \\
{\left[1-\left(1-\prod_{j=1}^{n}\left(1-\left(1-v_{\dot{\alpha}_{i \sigma(j)}^{-}}\right)^{\lambda}\right)^{\xi_{j}}\right)^{1 / \lambda},\right.} \\
\left.\dot{\alpha}_{i \sigma(1)} \in \dot{\tilde{a}}_{i \sigma(1)}, \dot{\alpha}_{i \sigma(2)} \in \dot{\tilde{a}}_{i \sigma(2)}, \ldots, \dot{\alpha}_{i \sigma(n)} \in \dot{\tilde{a}}_{i \sigma(n)}\right\}, \\
i=1,2, \ldots, m,
\end{gathered}
$$


or the GIVIHFHG operator (91):

$$
\begin{aligned}
& \tilde{a}_{i}=\operatorname{GIVIHFHG}_{\lambda}\left(\tilde{a}_{i 1}, \tilde{a}_{i 2}, \ldots, \tilde{a}_{i n}\right) \\
& =\left\{\left(\left[1-\left(1-\prod_{j=1}^{n}\left(1-\left(1-\mu_{\alpha_{i \sigma(j)}}^{-}\right)^{\lambda}\right)^{\xi_{j}}\right)^{1 / \lambda},\right.\right.\right. \\
& \left.1-\left(1-\prod_{j=1}^{n}\left(1-\left(1-\mu_{\dot{\alpha}_{i \sigma(j)}^{+}}\right)^{\lambda}\right)^{\xi_{j}}\right)^{1 / \lambda}\right], \\
& {\left[\left(1-\prod_{j=1}^{n}\left(1-\left(\nu_{\ddot{\alpha}_{i \sigma(j)}^{-}}^{-}\right)^{\lambda}\right)^{\xi_{j}}\right)^{1 / \lambda},\right.} \\
& \left.\left.\left(1-\prod_{j=1}^{n}\left(1-\left(v_{\dot{\alpha}_{i \sigma(j)}^{+}}^{+}\right)^{\lambda}\right)^{\xi_{j}}\right)^{1 / \lambda}\right]\right) \mid \\
& \left.\ddot{\alpha}_{i \sigma(1)} \in \ddot{\tilde{a}}_{i \sigma(1)}, \ddot{\alpha}_{i \sigma(2)} \in \ddot{\tilde{a}}_{i \sigma(2)}, \ldots, \ddot{\alpha}_{i \sigma(n)} \in \ddot{\tilde{a}}_{i \sigma(n)}\right\} \text {, } \\
& i=1,2, \ldots, m \text {, }
\end{aligned}
$$

to aggregate all the preference values $\tilde{a}_{i j}(j=1,2, \ldots, n)$ in the $i$ th line of $\widetilde{A}$, and then derive the collective overall preference value $\widetilde{a}_{i}=\left\{\alpha_{i} \mid \alpha_{i} \in \widetilde{a}_{i}\right\}=\left\{\left(\left[\mu_{\alpha_{i}}^{-}, \mu_{\alpha_{i}}^{+}\right],\left[\nu_{\alpha_{i}}^{-}, \nu_{\alpha_{i}}^{+}\right]\right) \mid\right.$ $\left.\alpha_{i} \in \tilde{a}_{i}\right\}(i=1,2, \ldots, m)$ of the alternative $Y_{i}(i=1,2, \ldots, m)$, where $\xi=\left(\xi_{1}, \xi_{2}, \ldots, \xi_{n}\right)^{T}$ is the associated weight vector of the GIVIHFHA (or GIVIHFHG) operator, with $\xi_{j} \in[0,1]$, $j=1,2, \ldots, n$, and $\sum_{j=1}^{n} \xi_{j}=1$.

Step 4. According to Definition 10, we calculate the score values $s\left(\widetilde{a}_{i}\right)(i=1,2, \ldots, m)$ of $\tilde{a}_{i}(i=1,2, \ldots, m)$ :

$$
s\left(\widetilde{a}_{i}\right)=\frac{(1 / 2) \sum_{\alpha_{i} \in \widetilde{a}_{i}}\left(\mu_{\alpha_{i}}^{-}-\nu_{\alpha_{i}}^{-}+\mu_{\alpha_{i}}^{+}-\nu_{\alpha_{i}}^{+}\right)}{\#\left(\tilde{a}_{i}\right)} .
$$

Step 5. Get the priority of the alternatives $Y_{i}(i=1,2, \ldots, m)$ by ranking $s\left(\tilde{a}_{i}\right) \quad(i=1,2, \ldots, m)$.

Step 6. End.

\section{The Application of the Developed Approach in Group Decision-Making Problems}

5.1. An Illustrative Example. In the following, we use a practical example to illustrate the application of the approach proposed in Section 4.

Example 1 (see [18]). Let us consider a factory which intends to select a new site for new buildings. Three alternatives $Y_{i}$ $(i=1,2,3)$ are available, and the three decision makers $D_{k}$ $(k=1,2,3)$ consider three criteria to decide which site to choose: (1) $G_{1}$ (price); (2) $G_{2}$ (location); and (3) $G_{3}$ (environment). Among the considered attributes, $G_{1}$ is of cost type, and $G_{j}(j=2,3)$ are of benefit type. The weight vector of the decision makers $D_{k}(k=1,2,3)$ is $\omega=$ $(0.1,0.6,0.3)^{T}$. The weight vector of the criteria $G_{j}(j=1$, $2,3)$ is $w=(0.5,0.2,0.3)^{T}$. Suppose that each decision maker provides his/her own interval-valued intuitionistic hesitant fuzzy decision matrix $\widetilde{R}^{(k)}=\left(\widetilde{r}_{i j}^{(k)}\right)_{3 \times 3}(k=1,2,3)$ as listed in Tables 2, 3, and 4, where $\tilde{r}_{i j}^{(k)}=\left\{\gamma_{i j}^{(k)} \mid \gamma_{i j}^{(k)} \in \widetilde{r}_{i j}^{(k)}\right\}$ is an IVIHFE given by the decision maker $D_{k}$.

Step 1. Based on (102), we transform the interval-valued intuitionistic hesitant fuzzy decision matrices $\widetilde{R}^{(k)}=\left(\widetilde{r}_{i j}^{(k)}\right)_{3 \times 3}(k=$ $1,2,3)$ into the normalized matrices $\widetilde{A}^{(k)}=\left(\widetilde{a}_{i j}^{(k)}\right)_{3 \times 3}(k=1$, 2,3) (see Tables 5, 6, and 7).

Step 2. Let $\lambda=6$, and utilize the GIVIHFWA operator (104) to aggregate all the individual interval-valued intuitionistic hesitant fuzzy decision matrices $\widetilde{A}^{(k)}=\left(\widetilde{a}_{i j}^{(k)}\right)_{3 \times 3}(k=1,2,3)$ into the collective interval-valued intuitionistic hesitant fuzzy decision matrix $\widetilde{A}=\left(\widetilde{a}_{i j}\right)_{3 \times 3}$ (see Table 8 ).

Step 3. Utilize the GIVIHFHA operator (106) (whose associated weighting vector is $\xi=(0.25,0.65,0.1)^{T}$ and $\left.\lambda=6\right)$ to aggregate all the preference values $\tilde{a}_{i j}(j=1,2,3)$ in the $i$ th line of $\widetilde{A}$, and then derive the collective overall preference value $\tilde{a}_{i}(i=1,2,3)$ of the alternative $Y_{i}(i=1,2,3)$. We will not list the collective overall preference values here because of space limitations.

Step 4. According to (108), we calculate the score values $s\left(\widetilde{a}_{i}\right)$ $(i=1,2,3)$ of $\widetilde{a}_{i}(i=1,2,3)$ :

$$
\begin{gathered}
s\left(\widetilde{a}_{1}\right)=0.4314, \quad s\left(\widetilde{a}_{2}\right)=0.3990, \\
s\left(\widetilde{a}_{3}\right)=0.4852 .
\end{gathered}
$$

Step 5. By $\left(\tilde{a}_{i}\right)(i=1,2,3)$, rank all the alternatives $Y_{i}(i=$ $1,2,3)$ in descending order:

$$
Y_{3}>Y_{1}>Y_{2}
$$

Thus, the best alternative is $Y_{3}$.

As the parameter $\lambda$ changes, we can get different results (see Table 9). From Table 9, we can find that the score values obtained by the GIVIHFWA and GIVIHFHA operators become bigger as the parameter $\lambda$ increases for the same aggregation arguments, and the decision makers can choose the values of $\lambda$ according to their preferences.

Furthermore, it is possible to analyze how the different attitudinal character $\lambda$ plays a role in the aggregation results; in this case, we consider different values of $\lambda$ : $0.0001,0.0002,0.0003,0.0004,0.0005, \ldots, 50$, which are provided by the decision maker. The score functions $s\left(\widetilde{a}_{i}\right)(i=$ $1,2,3)$ of the collective overall preference values $\tilde{a}_{i}(i=$ $1,2,3)$ of the alternatives $Y_{i}(i=1,2,3)$ are shown in Figure 1.

It is observed from Figure 1 that all of $s\left(\widetilde{a}_{i}\right)(i=1,2,3)$ increase as $\lambda$ increases. From Figure 1, we can find that

(1) when $\lambda \in(0,1.4234)$, the ranking of the three alternatives is $Y_{3}>Y_{2}>Y_{1}$ and the best choice is $Y_{3}$; 
TABLE 2: The interval-valued intuitionistic hesitant fuzzy decision matrix $\widetilde{R}^{(1)}$ provided by $D_{1}$.

\begin{tabular}{lccc}
\hline $\mathbf{2}$ & $G_{1}$ & $G_{2}$ & $G_{3}$ \\
\hline$Y_{1}$ & $\{([0.4,0.5],[0.3,0.5])\}$ & $\{([0.2,0.3],[0.6,0.7])\}$ & $\begin{array}{c}\{([0.6,0.8],[0.1,0.2]), \\
([0.6,0.7],[0.3,0.3]), \\
([0.7,0.9],[0.1,0.1]\}\end{array}$ \\
\hline$Y_{2}$ & $\begin{array}{l}\{([0.1,0.1],[0.8,0.9]), \\
([0.2,0.4],[0.5,0.6])\}\end{array}$ & $\begin{array}{c}([0.4,0.5],[0.5,0.5]), \\
([0.6,0.7],[0.1,0.2]),\end{array}$ \\
\hline$Y_{3}$ & $\begin{array}{l}\{([0.2,0.3],[0.6,0.6]), \\
([0.1,0.3],[0.6,0.7])\}\end{array}$ & $\{([0.8,0.9],[0.1,0.1])\}$ & $\{([0.8,0.9],[0.1,0.1])\}$ \\
\hline
\end{tabular}

TABLE 3: The interval-valued intuitionistic hesitant fuzzy decision matrix $\widetilde{R}^{(2)}$ provided by $D_{2}$.

\begin{tabular}{lcrr}
\hline 3 & $G_{1}$ & $G_{2}$ & $G_{3}$ \\
\hline & $\begin{array}{c}\{([0.7,0.9],[0.1,0.1]), \\
([0.7,0.8],[0.1,0.2]), \\
Y_{1}\end{array}$ & $\begin{array}{c}\{([0.4,0.6],[0.3,0.3]), \\
([0.5,0.7],[0.2,0.3])\}\end{array}$ & $\{([0.9,0.9],[0.1,0.1])\}$ \\
\hline$Y_{2}$ & $\{([0.7,0.8],[0.1,0.2])\}$ & $\{([0.8,0.9],[0.1,0.1])\}$ & $\begin{array}{c}([0.5,0.7],[0.2,0.3]), \\
([0.6,0.8],[0.1,0.2])\}\end{array}$ \\
& & $\{([0.6,0.8],[0.2,0.2])$, & $\{([0.1,0.3],[0.6,0.7])$, \\
$Y_{3}$ & $\{([0.2,0.3],[0.5,0.6])\}$ & $([0.7,0.9],[0.1,0.1])\}$ & $(0.2,0.2],[0.7,0.8])$, \\
& & & $([0.3,0.4],[0.6,0.6])\}$ \\
\hline
\end{tabular}

TABLe 4: The interval-valued intuitionistic hesitant fuzzy decision matrix $\widetilde{R}^{(3)}$ provided by $D_{3}$.

\begin{tabular}{lccc}
\hline $\mathbf{4}$ & $G_{1}$ & $G_{2}$ & $G_{3}$ \\
\hline$Y_{1}$ & $\{([0.3,0.5],[0.4,0.5])\}$ & $\begin{array}{c}\{([0.2,0.3],[0.5,0.6]), \\
([0.1,0.1],[0.8,0.9])\}\end{array}$ & $\{([0.7,0.8],[0.2,0.2])\}$ \\
\hline$Y_{2}$ & $\{([0.4,0.6],[0.3,0.4])$, & & $\{([0.3,0.5],[0.5,0.5])$, \\
& $([0.4,0.5],[0.5,0.5])\}$ & $\{([0.1,0.3],[0.6,0.6])\}$ & $([0.1,0.2],[0.7,0.7])$, \\
$([0.3,0.4],[0.5,0.6])\}$ & \\
& & & $\{([0.9,0.9],[0.1,0.1])\}$ \\
$Y_{3}$ & $([0.8,0.9],[0.1,0.1])$, & $([0.3,0.6],[0.4,0.4])$, & $(0.4,0.5],[0.4,0.5])$, \\
& $([0.5,0.7],[0.1,0.2])\}$ & $([0.3,0.5],[0.4,0.5])\}$ & \\
\hline
\end{tabular}

TABLE 5: The interval-valued intuitionistic hesitant fuzzy decision matrix $\widetilde{A}^{(1)}$.

\begin{tabular}{lccc}
\hline $\mathbf{5}$ & $G_{1}$ & $G_{2}$ & $G_{3}$ \\
\hline$Y_{1}$ & $\{([0.3,0.5],[0.4,0.5])\}$ & $\{([0.2,0.3],[0.6,0.7])\}$ & $\begin{array}{c}\{(0.6,0.8],[0.1,0.2]), \\
([0.6,0.7],[0.3,0.3]), \\
([0.7,0.9],[0.1,0.1]\}\end{array}$ \\
\hline$Y_{2}$ & $\begin{array}{l}\{([0.8,0.9],[0.1,0.1]), \\
([0.5,0.6],[0.2,0.4])\}\end{array}$ & $\begin{array}{c}\{([0.4,0.5],[0.5,0.5]), \\
([0.6,0.7],[0.1,0.2]), \\
([0.5,0.7],[0.1,0.2])\}\end{array}$ & $([0.8,0.9],[0.1,0.1])$ \\
\hline$Y_{3}$ & $\begin{array}{c}\{([0.6,0.6],[0.2,0.3]), \\
([0.6,0.7],[0.1,0.3])\}\end{array}$ & $\{([0.8,0.9],[0.1,0.1])\}$ & $\{([0.3,0.5],[0.4,0.4])\}$ \\
\hline
\end{tabular}

TABLE 6: The interval-valued intuitionistic hesitant fuzzy decision matrix $\widetilde{A}^{(2)}$.

\begin{tabular}{cccc}
\hline $\mathbf{6}$ & $G_{1}$ & $G_{2}$ & $G_{3}$ \\
\hline$Y_{1}$ & $\begin{array}{c}\{([0.1,0.1],[0.7,0.9]), \\
([0.1,0.2],[0.7,0.8]), \\
([0.1,0.1],[0.6,0.8])\}\end{array}$ & $\begin{array}{c}\{([0.4,0.6],[0.3,0.3]), \\
([0.5,0.7],[0.2,0.3])\}\end{array}$ & $\{([0.9,0.9],[0.1,0.1])\}$ \\
\hline$Y_{2}$ & $\{([0.1,0.2],[0.7,0.8])\}$ & $\{([0.8,0.9],[0.1,0.1])\}$ & $\begin{array}{c}\{([0.5,0.7],[0.2,0.3]), \\
([0.6,0.8],[0.1,0.2])\}\end{array}$ \\
\hline & & $\{([0.6,0.8],[0.2,0.2])$, & $\{([0.1,0.3],[0.6,0.7])$, \\
$Y_{3}$ & $\{([0.5,0.6],[0.2,0.3])\}$ & $([0.7,0.9],[0.1,0.1])\}$ & $(0.2,0.2],[0.7,0.8])$, \\
& & & $([0.3,0.4],[0.6,0.6])\}$ \\
\hline
\end{tabular}


TABLE 7: The interval-valued intuitionistic hesitant fuzzy decision matrix $\widetilde{A}^{(3)}$.

\begin{tabular}{cccc}
\hline 7 & $G_{1}$ & $G_{2}$ & $G_{3}$ \\
\hline$Y_{1}$ & $\{([0.4,0.5],[0.3,0.5])\}$ & $\begin{array}{c}\{([0.2,0.3],[0.5,0.6]), \\
([0.1,0.1],[0.8,0.9])\}\end{array}$ & $\{([0.7,0.8],[0.2,0.2])\}$ \\
\hline & & & $\{([0.3,0.5],[0.5,0.5])$, \\
$Y_{2}$ & $\{([0.3,0.4],[0.4,0.6])$, & $\{([0.1,0.3],[0.6,0.6])\}$ & $([0.3,0.2],[0.7,0.7])$, \\
& $([0.5,0.5],[0.4,0.5])\}$ & & $\{0.5,0.6])\}$ \\
\hline & $\{([0.1,0.1],[0.8,0.9])$, & $([0.4,0.5],[0.4,0.5])$, & $\{([0.9,0.9],[0.1,0.1])\}$ \\
$Y_{3}$ & $([0.1,0.2],[0.5,0.7])\}$ & $([0.3,0.5],[0.4,0.5])\}$ & \\
\hline
\end{tabular}

TABLE 8: The collective interval-valued intuitionistic hesitant fuzzy decision matrix $\widetilde{A}$.

\begin{tabular}{|c|c|c|c|}
\hline 8 & $G_{1}$ & $G_{2}$ & $G_{3}$ \\
\hline$Y_{1}$ & $\begin{array}{c}\{([0.3305,0.4295],[0.4107,0.5705]) \\
([0.3305,0.4300],[0.4107,0.5700]) \\
([0.3305,0.4295],[0.4063,0.5700])\}\end{array}$ & $\begin{array}{c}\{([0.3680,0.5528],[0.3476,0.3527]) \\
([0.3676,0.5521],[0.3539,0.3544]) \\
([0.4596,0.6459],[0.2550,0.3527]) \\
([0.4595,0.6456],[0.2578,0.3544])\}\end{array}$ & $\begin{array}{c}\{([0.8554,0.8715],[0.1204,0.1285]), \\
([0.8554,0.8681],[0.1319,0.1319]), \\
([0.8571,0.8796],[0.1204,0.1204])\}\end{array}$ \\
\hline$Y_{2}$ & $\begin{array}{l}\{([0.5579,0.6482],[0.3349,0.3518]) \\
([0.5706,0.6528],[0.3349,0.3472]) \\
([0.3485,0.4266],[0.4055,0.5734]) \\
([0.4295,0.4602],[0.4055,0.5398])\}\end{array}$ & $\begin{array}{l}\{([0.7421,0.8460],[0.1538,0.1538]) \\
([0.7448,0.8486],[0.1372,0.1470]) \\
([0.7429,0.8486],[0.1372,0.1470])\}\end{array}$ & $\begin{array}{c}([0.5830,0.7241],[0.2166,0.2759]), \\
([0.5825,0.7207],[0.2185,0.2792]), \\
([0.5830,0.7216],[0.2166,0.2784]), \\
([0.6214,0.7834],[0.1365,0.2166]), \\
([0.6210,0.7814],[0.1374,0.2185]), \\
([0.6214,0.7819],[0.1365,0.2181])\}\end{array}$ \\
\hline$Y_{3}$ & $\begin{array}{c}\{([0.4917,0.5660],[0.2407,0.3384]) \\
([0.4917,0.5661],[0.2382,0.3381]) \\
([0.4917,0.5856],[0.2186,0.3384]) \\
([0.4917,0.5857],[0.2166,0.3381])\}\end{array}$ & $\begin{array}{l}\{([0.6214,0.7875],[0.2125,0.2125]), \\
([0.6231,0.7834],[0.2125,0.2166]), \\
([0.6214,0.7834],[0.2125,0.2166]), \\
([0.6817,0.8654],[0.1346,0.1346]) \\
([0.6827,0.8635],[0.1346,0.1365]), \\
([0.6817,0.8635],[0.1346,0.1365])\}\end{array}$ & $\begin{array}{c}\{([0.7669,0.7679],[0.2295,0.2305]), \\
([0.7669,0.7677],[0.2305,0.2307]), \\
([0.7671,0.7689],[0.2295,0.2295])\}\end{array}$ \\
\hline
\end{tabular}

(2) when $\lambda \in[1.4234,22.2700]$, the ranking of the three alternatives is $Y_{3}>Y_{1}>Y_{2}$ and the best choice is $Y_{3}$;

(3) when $\lambda \in(22.2700,37.7207)$, the ranking of the three alternatives is $Y_{3}>Y_{2}>Y_{1}$ and the best choice is $Y_{3}$;

(4) when $\lambda \in[37.7207,50]$, the ranking of the three alternatives is $Y_{2}>Y_{3}>Y_{1}$ and the best choice is $Y_{2}$.

In Steps 2 and 3, instead of the GIVIHFWA and GIVIHFHA operators, if we use the GIVIHFWG and GIVIHFHG operators to aggregate the values of the alternatives, the score values and the rankings of the alternatives are listed in Table 10. By Table 10, we can find that the score values obtained by the GIVIHFWG and GIVIHFHG operators become smaller as the parameter $\lambda$ increases for the same aggregation arguments, and the decision makers can choose the values of $\lambda$ according to their preferences.

Furthermore, it is possible to analyze how the different attitudinal character $\lambda$ plays a role in the aggregation results; in this case, we consider different values of $\lambda$ : $0.0001,0.0002,0.0003,0.0004,0.0005, \ldots, 50$, which are provided by the decision maker. The score functions $s\left(\widetilde{a}_{i}\right)(i=$ $1,2,3)$ of the collective overall preference values $\tilde{a}_{i}(i=$ $1,2,3)$ of the alternatives $Y_{i}(i=1,2,3)$ are shown in Figure 2.

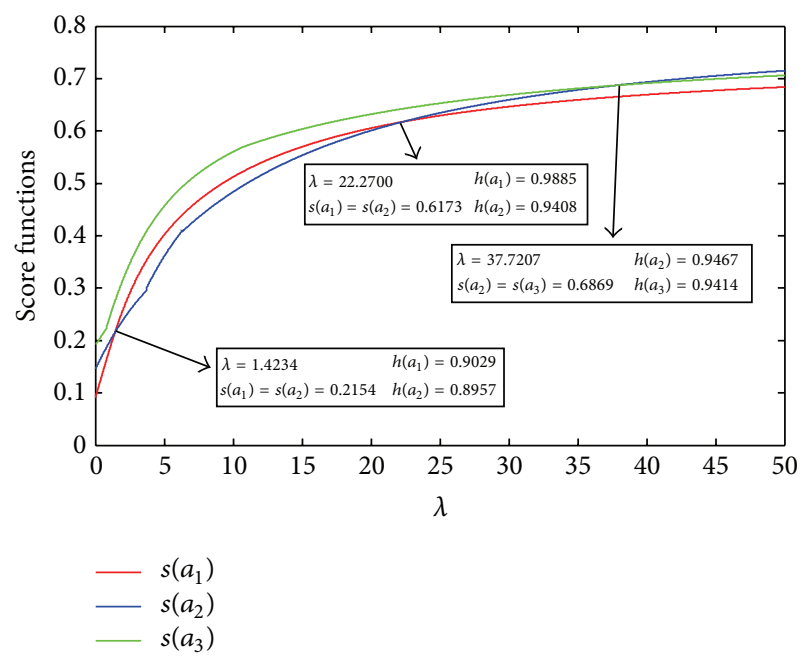

Figure 1: Variation of the score functions with respect to the parameter $\lambda$.

It is observed from Figure 2 that all of $s\left(\widetilde{a}_{i}\right)(i=1,2,3)$ decrease as $\lambda$ increases. From Figure 2, we can find that

(1) when $\lambda \in(0,6.1065)$, the ranking of the three alternatives is $Y_{2}>Y_{1}>Y_{3}$ and the best choice is $Y_{2}$; 
TABLE 9: Score values obtained by the GIVIHFWA and GIVIHFHA operators and the rankings of alternatives.

\begin{tabular}{lcccccc}
\hline $\mathbf{9}$ & $\lambda=0.1$ & $\lambda=10$ & $\lambda=20$ & $\lambda=30$ & $\lambda=40$ & $\lambda=50$ \\
\hline$Y_{1}$ & 0.0994 & 0.5120 & 0.6054 & 0.6464 & 0.6693 & 0.6839 \\
$Y_{2}$ & 0.1512 & 0.4834 & 0.6006 & 0.6593 & 0.6933 & 0.7151 \\
$Y_{3}$ & 0.1946 & 0.5602 & 0.6320 & 0.6688 & 0.6912 & 0.7061 \\
\hline Ranking & $Y_{3}>Y_{2}>Y_{1}$ & $Y_{3}>Y_{1}>Y_{2}$ & $Y_{3}>Y_{1}>Y_{2}$ & $Y_{3}>Y_{2}>Y_{1}$ & $Y_{2}>Y_{3}>Y_{1}$ & $Y_{2}>Y_{3}>Y_{1}$ \\
\hline
\end{tabular}

TABLE 10: Score values obtained by the GIVIHFWG and GIVIHFHG operators and the rankings of alternatives.

\begin{tabular}{lcccccc}
\hline $\mathbf{1 0}$ & $\lambda=0.1$ & $\lambda=10$ & $\lambda=20$ & $\lambda=30$ & $\lambda=40$ & $\lambda=50$ \\
\hline$Y_{1}$ & 0.2199 & -0.4576 & -0.6175 & -0.6812 & -0.7152 & -0.7363 \\
$Y_{2}$ & 0.2350 & -0.4422 & -0.6012 & -0.6656 & -0.7001 & -0.7216 \\
$Y_{3}$ & 0.1199 & -0.4236 & -0.5704 & -0.6359 & -0.6724 & -0.6953 \\
\hline Ranking & $Y_{2}>Y_{1}>Y_{3}$ & $Y_{3}>Y_{2}>Y_{1}$ & $Y_{3}>Y_{2}>Y_{1}$ & $Y_{3}>Y_{2}>Y_{1}$ & $Y_{3}>Y_{2}>Y_{1}$ & $Y_{3}>Y_{2}>Y_{1}$ \\
\hline
\end{tabular}

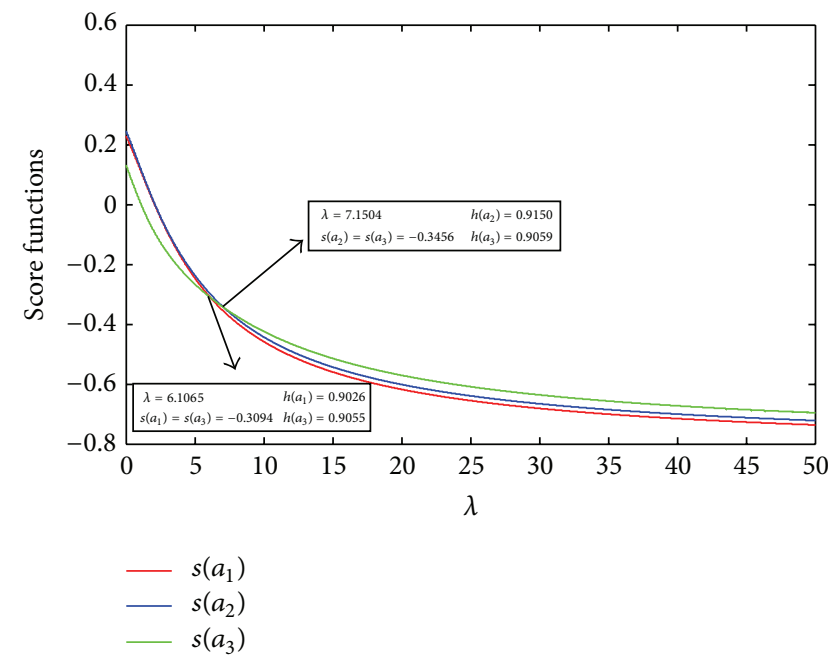

FIGURE 2: Variation of the score functions with respect to the parameter $\lambda$.

(2) when $\lambda \in[6.1065,7.1504]$, the ranking of the three alternatives is $Y_{2}>Y_{3}>Y_{1}$ and the best choice is $Y_{2}$;

(3) when $\lambda \in(7.1504,50]$, the ranking of the three alternatives is $Y_{3}>Y_{2}>Y_{1}$ and the best choice is $Y_{3}$.

\subsection{Comparison with the Existing Hesitant Fuzzy Aggregation} Operators and MAGDM Methods. In the following, we compare our operators and methods with the existing aggregation operators and methods to demonstrate the advantages of the operators and methods proposed here.

Because interval-valued intuitionistic hesitant fuzzy set is a substantial and important generalization of hesitant fuzzy set, interval-valued hesitant fuzzy set, and intuitionistic hesitant fuzzy set, the developed interval-valued intuitionistic hesitant fuzzy aggregation operators are substantial and important generalizations of the existing hesitant fuzzy aggregation operators [9], interval-valued hesitant fuzzy aggregation operators [6], and intuitionistic hesitant fuzzy aggregation operators ((67), (68), (69), (70), (80), (81), (82), (83),
(98), (99), (100), and (101)). Thus, the developed intervalvalued intuitionistic hesitant fuzzy MAGDM method is a substantial and important generalization of the existing hesitant fuzzy MAGDM method [9], interval-valued hesitant fuzzy MAGDM method [6], and generalized hesitant fuzzy MAGDM method [7]. Concretely, our operators and methods can be applied to decision-making problems in which the attribute values take the form of hesitant fuzzy elements, interval-valued hesitant fuzzy elements, and intuitionistic hesitant fuzzy elements. In contrast, the existing hesitant fuzzy aggregation operators and MAGDM method, intervalvalued hesitant fuzzy aggregation operators and MAGDM method, and intuitionistic hesitant fuzzy aggregation operators and MAGDM method cannot be applied to decisionmaking problems in which the attribute values are given in the form of interval-valued intuitionistic hesitant fuzzy elements. In other words, our operators and methods have much wider applications than the existing hesitant fuzzy aggregation operators and MAGDM method, interval-valued hesitant fuzzy aggregation operators and MAGDM method, and intuitionistic hesitant fuzzy aggregation operators and MAGDM method. For example, Example 1 cannot be handled by the existing hesitant fuzzy aggregation operators and MAGDM method, interval-valued hesitant fuzzy aggregation operators and MAGDM method, and intuitionistic hesitant fuzzy aggregation operators and MAGDM method, whereas our operators and methods can deal with Example 4 in [9], Example 6 in [6], and Example 4 in [7].

5.3. Comparison with the Existing Intuitionistic Fuzzy Aggregation Operators and MAGDM Methods. Considering that interval-valued intuitionistic hesitant fuzzy set is a substantial and important generalization of intuitionistic fuzzy set and interval-valued intuitionistic fuzzy set, the developed interval-valued intuitionistic hesitant fuzzy aggregation operators and MAGDM method are substantial and important generalizations of the existing intuitionistic fuzzy aggregation operators and MAGDM methods and the existing interval-valued intuitionistic fuzzy aggregation operators and MAGDM methods. In other words, our operators and methods can be applied to decision-making problems 
in which the attribute values are in the form of intuitionistic fuzzy numbers and interval-valued intuitionistic fuzzy numbers, whereas the existing intuitionistic fuzzy aggregation operators and MAGDM methods and the existing interval-valued intuitionistic fuzzy aggregation operators and MAGDM methods have difficulty in dealing with decision-making problems in which the attribute values are given in the form of interval-valued intuitionistic hesitant fuzzy elements. In short, our operators and methods have much wider applications than the existing intuitionistic fuzzy aggregation operators and MAGDM methods and the existing interval-valued intuitionistic fuzzy aggregation operators and MAGDM methods.

\section{Conclusions}

In this paper, we first propose the concept of interval-valued intuitionistic hesitant fuzzy sets, discuss their some basic properties, and develop some operational rules for intervalvalued intuitionistic hesitant fuzzy elements. Then, we focus on interval-valued intuitionistic hesitant fuzzy information aggregation techniques and propose a series of intervalvalued intuitionistic hesitant fuzzy aggregation operators. Moreover, we apply the developed aggregation operators to multiple attribute group decision-making with intervalvalued intuitionistic hesitant fuzzy information. Finally, a numerical example is used to illustrate the validity of the proposed approach in group decision-making problems.

In group decision-making problems, because the experts usually come from different specialty fields and have different backgrounds and levels of knowledge, they usually have diverging opinions. Therefore, how to obtain the maximum degree of consensus or agreement from these experts for the given alternatives is an interesting and important research topic which has been receiving more and more attention in recent years. However, there are not similar studies completed for group decision-makings with interval-valued intuitionistic hesitant fuzzy information. Thus, in future work, we will present a consensus model for group decision-making with interval-valued intuitionistic hesitant fuzzy information.

\section{Acknowledgments}

The authors thank the anonymous referees for their valuable suggestions for improving this paper. This work was supported by the National Natural Science Foundation of China (Grant no. 61073121) and the Natural Science Foundation of the Hebei Province of China (Grant nos. F2012201014, F2013201060, and A2012201033).

\section{References}

[1] K. T. Atanassov, "Intuitionistic fuzzy sets," Fuzzy Sets and Systems, vol. 20, no. 1, pp. 87-96, 1986.

[2] L. Zadeh, "The concept of a linguistic variable and its application to approximate reasoning, Part 1," Information Sciences, vol. 8, pp. 199-249, 1975.

[3] D. Dubois and H. Prade, Fuzzy Sets and Systems: Theory and Applications, vol. 144 of Mathematics in Science and Engineering, Academic Press, New York, NY, USA, 1980.
[4] V. Torra, "Hesitant fuzzy sets," International Journal of Intelligent Systems, vol. 25, no. 6, pp. 529-539, 2010.

[5] L. A. Zadeh, "Fuzzy sets," Information and Computation, vol. 8, pp. 338-353, 1965.

[6] N. Chen, Z. S. Xu, and M. M. Xia, "Interval-valued hesitant preference relations and their applications to group decision making," Knowledge-Based Systems, vol. 37, pp. 528-540, 2013.

[7] G. Qian, H. Wang, and X. Q. Feng, "Generalized hesitant fuzzy sets and their application in decision support system," Knowledge-Based Systems, vol. 37, pp. 357-365, 2013.

[8] K. Atanassov and G. Gargov, "Interval valued intuitionistic fuzzy sets," Fuzzy Sets and Systems, vol. 31, no. 3, pp. 343-349, 1989.

[9] M. Xia and Z. Xu, "Hesitant fuzzy information aggregation in decision making," International Journal of Approximate Reasoning, vol. 52, no. 3, pp. 395-407, 2011.

[10] Z.-S. Xu, "Methods for aggregating interval-valued intuitionistic fuzzy information and their application to decision making," Kongzhi yu Juece/Control and Decision, vol. 22, no. 2, pp. 215219, 2007.

[11] Z. Xu and R. R. Yager, "Some geometric aggregation operators based on intuitionistic fuzzy sets," International Journal of General Systems, vol. 35, no. 4, pp. 417-433, 2006.

[12] H. Dyckhoff and W. Pedrycz, "Generalized means as model of compensative connectives," Fuzzy Sets and Systems, vol. 14, no. 2, pp. 143-154, 1984.

[13] Z. M. Zhang, "Hesitant fuzzy power aggregation operators and their application to multiple attribute group decision making," Information Sciences, vol. 234, pp. 150-181, 2013.

[14] V. Torra and Y. Narukawa, Modeling Decisions: Information Fusion and Aggregation Operators, Springer, 2007.

[15] Z. Xu, "On consistency of the weighted geometric mean complex judgement matrix in AHP," European Journal of Operational Research, vol. 126, no. 3, pp. 683-687, 2000.

[16] Z. Xu and X. Cai, "Nonlinear optimization models for multiple attribute group decision making with intuitionistic fuzzy information," International Journal of Intelligent Systems, vol. 25, no. 6, pp. 489-513, 2010.

[17] Z. Xu and H. Hu, "Projection models for intuitionistic fuzzy multiple attribute decision making," International Journal of Information Technology and Decision Making, vol. 9, no. 2, pp. 267-280, 2010.

[18] B. Zhu, Z. Xu, and M. Xia, "Hesitant fuzzy geometric Bonferroni means," Information Sciences, vol. 205, pp. 72-85, 2012. 


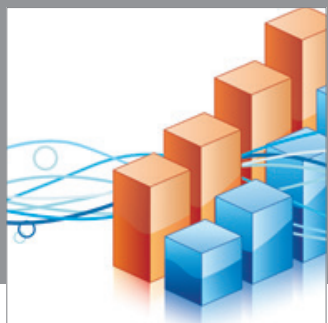

Advances in

Operations Research

mansans

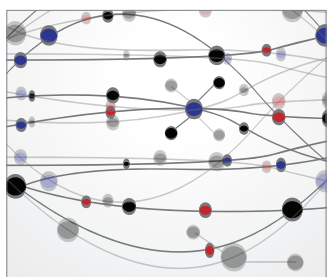

The Scientific World Journal
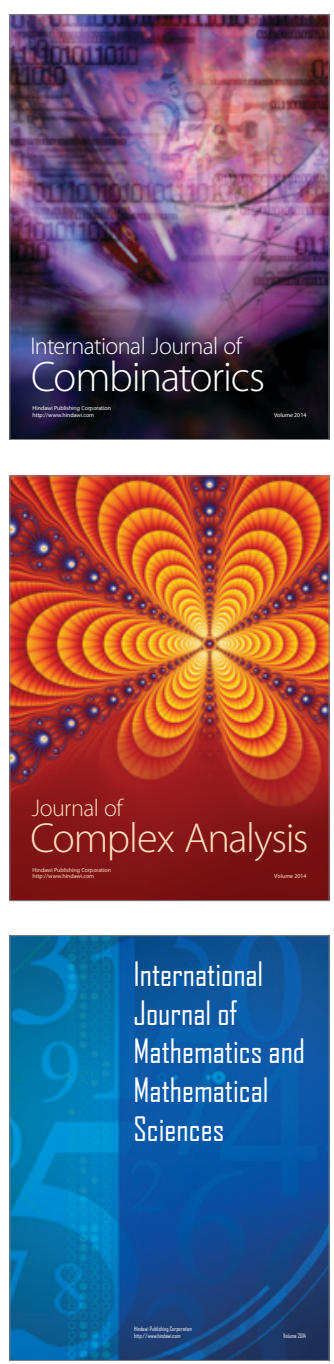
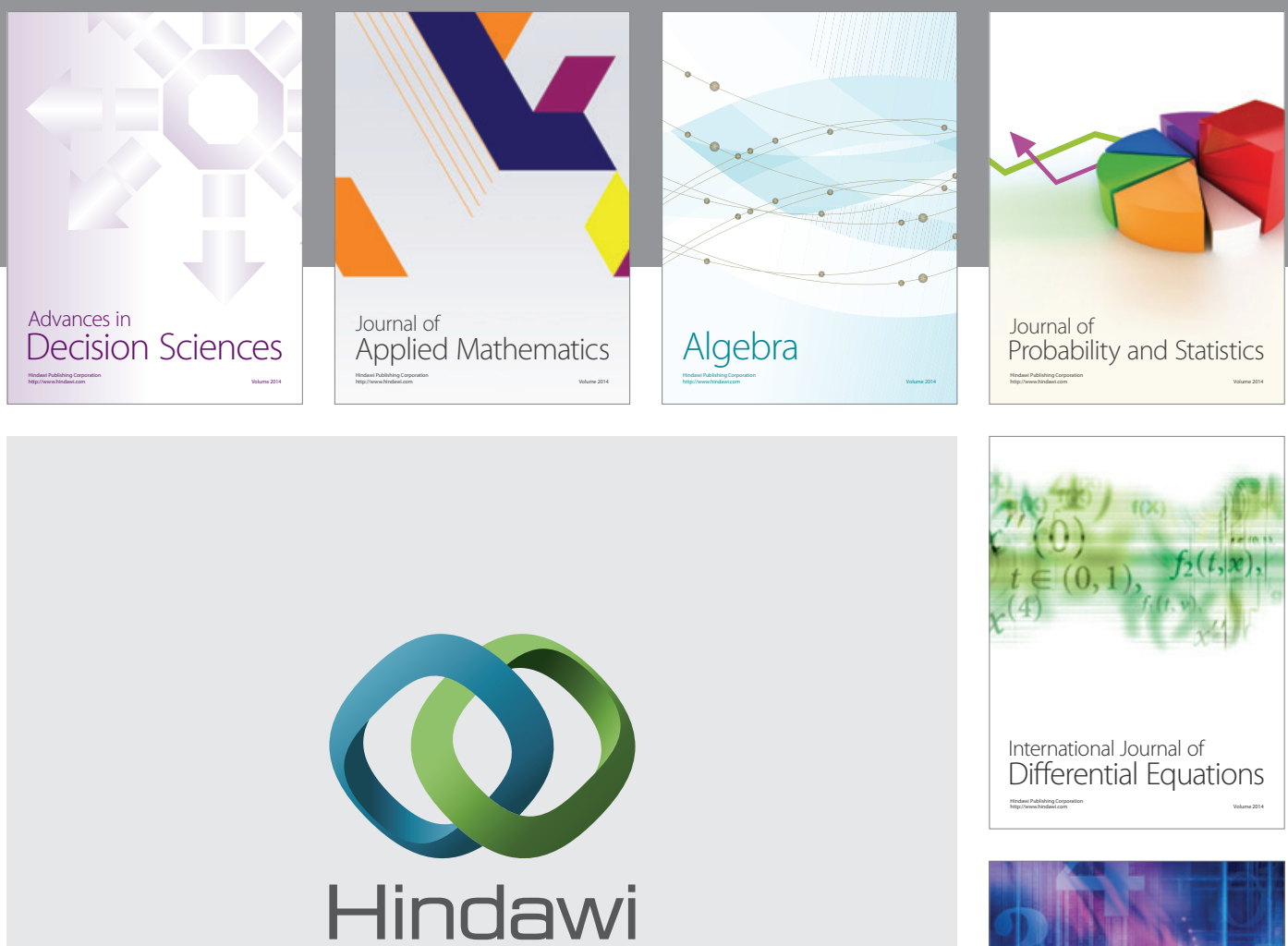

Submit your manuscripts at http://www.hindawi.com
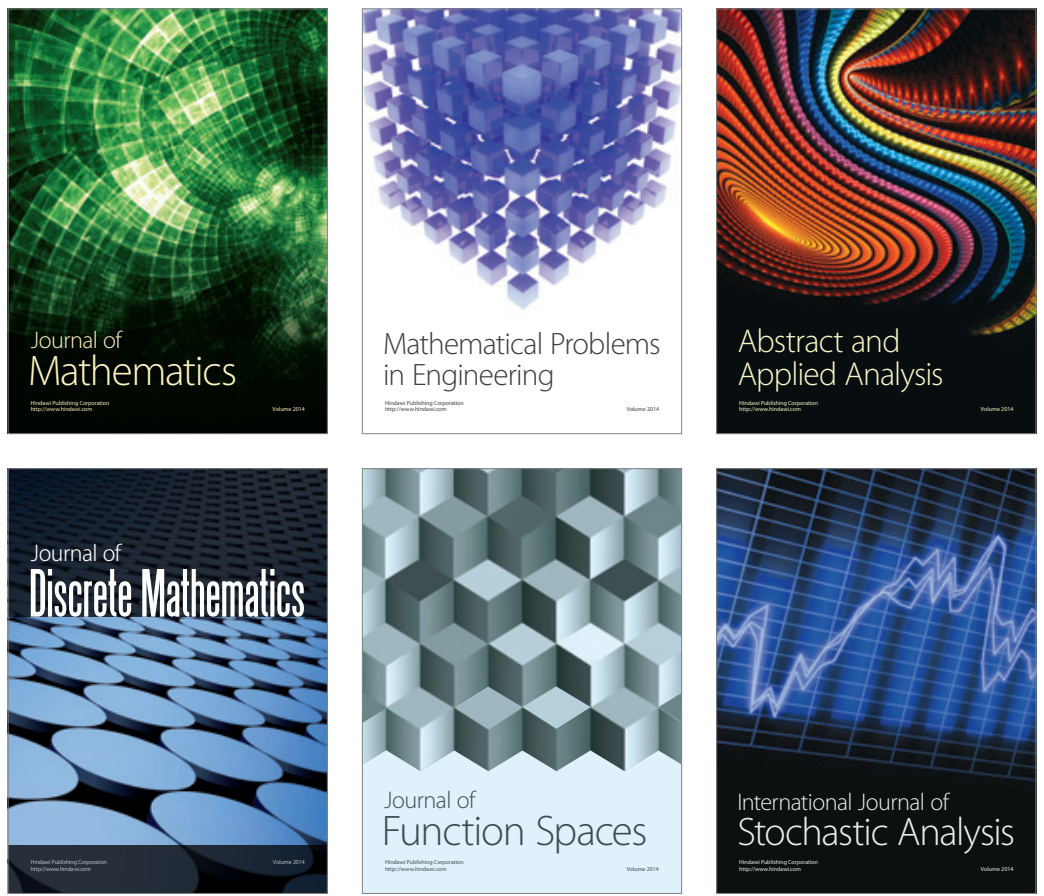

Journal of

Function Spaces

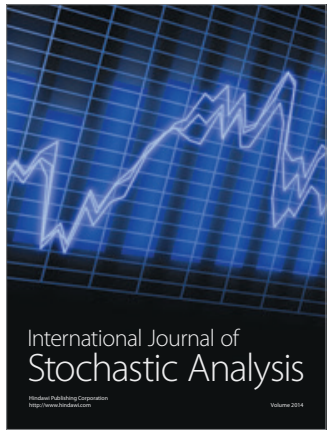

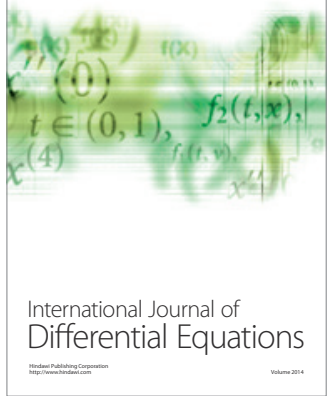
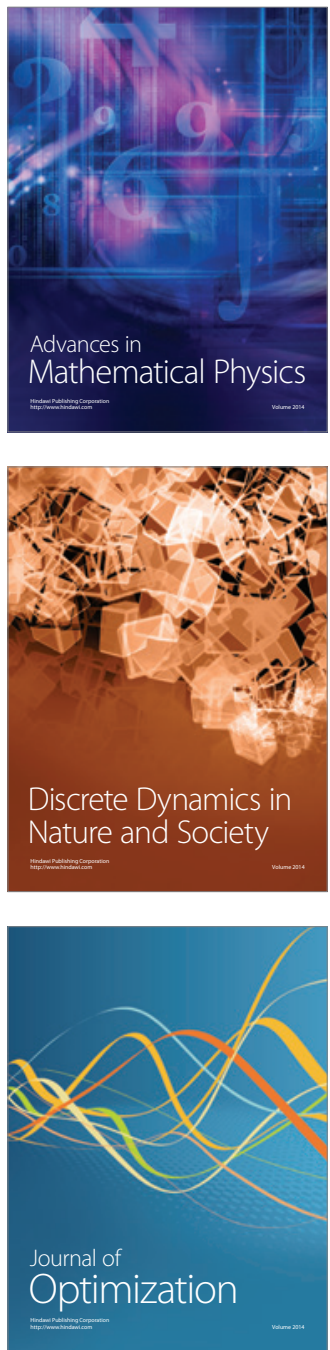